[American Journal of Science, Vol. 314, January, 2014, P. 110-153, DOI 10.2475/01.2014.04]

\title{
NEOPROTEROZOIC S-TYPE GRANITES IN THE ALXA BLOCK, WESTERNMOST NORTH CHINA AND TECTONIC IMPLICATIONS: IN SITU ZIRCON U-Pb-Hf-O ISOTOPIC AND GEOCHEMICAL CONSTRAINTS
}

\author{
WEI DAN***, XIAN-HUA LI*,§,†, QIANG WANG**, XUAN-CE WANG***, \\ and YU LIU*
}

\begin{abstract}
The Alxa Block in northern China has been traditionally considered as the westernmost part of the Archean North China Craton (NCC). However, recent studies revealed that there are few Archean rocks exposed in the Alxa Block, and the Paleoproterozoic geology of this block is different from that of the western part of the NCC. Thus, the tectonic affinity of the Alxa Block to the NCC and/or other Precambrian blocks needs further investigations. In this study, we carry out integrated analyses of in situ zircon $\mathrm{U}-\mathrm{Pb}$ age and $\mathrm{Hf}-\mathrm{O}$ isotopes as well as whole-rock geochemistry and Nd isotopes for the Neoproterozoic Dabusushan and Naimumaodao granites from central Alxa Block. Secondary ion mass spectrometry (SIMS) U-Pb zircon dating results indicate that the Naimumaodao and Dabusushan granite plutons were formed at $c a .930 \mathrm{Ma}$ and $c a .910 \mathrm{Ma}$, respectively. These granites are peraluminous $(\mathrm{A} / \mathrm{CNK}$ value $>1.0$ ), and contain peraluminous minerals such as muscovite and tourmaline, similar to those of S-type granites. They are characterized by high zircon $\delta^{18} \mathrm{O}$ values of ca. 8.2 to 12.1 permil, corresponding to a calculated magmatic $\delta^{18} \mathrm{O}$ value of $c a .10 .5$ to 14.3 permil, variable zircon $\varepsilon_{\mathrm{Hf}}(\mathrm{t})$ values of -6.2 to +3.8 (corresponding to $\mathrm{Hf}$ model ages of 2.2 to $1.6 \mathrm{Ga}$ ) and whole-rock $\varepsilon_{\mathrm{Nd}}(\mathrm{t})$ values of -10.1 to -4.5 (corresponding to $\mathrm{Nd}$ model ages of 2.4-1.9 Ga). The petrological and $\mathrm{Nd}-\mathrm{Hf}-\mathrm{O}$ isotopic study indicated that these granites were most probably generated by remelting of dominant (meta)sedimentary rocks in an orogenesis-related compressional environment. There is a clear contrast in the Precambrian geological evolution, including basement rock age data, Precambrian magmatism and detrital zircon age patterns, between the Alxa Block and the NCC. Furthermore, the new in-situ detrital zircon ages on Neoproterozoic (meta) sedimentary rock suggest that Alxa Block is likely related to the Cathaysia Block of South China during the Neoproterozoic, and amalgamated with the NCC since the Early Paleozoic. Thus, our new data suggest that the Alxa Block is most likely a separated Precambrian terrane from the Western Block of the NCC.
\end{abstract}

Key words: North China Craton, Alxa Block, Neoproterozoic, S-type granite, zircon Hf-O isotopes

INTRODUCTION

Understanding of the formation and evolution of the North China Craton (NCC) has been improved in the past decade by recognition of two Paleoproterozoic orogenic belts, that is, the Khondalite Belt (Zhao and others, 2005; Xia and others, 2006; Yin and others, 2009; Dan and others, 2012a) and the Trans-North China Orogen (TNCO) (Zhao and others, 2001, 2005). There is a general consensus that the NCC was formed at $c a .1 .85 \mathrm{Ga}$ (for example, Zhao and others, 2005; Zhai and Santosh, 2011), and it

* State Key Laboratory of Lithospheric Evolution, Institute of Geology and Geophysics, Chinese Academy of Sciences, Beijing 100029, China

** State Key Laboratory of Isotope Geochemistry, Guangzhou Institute of Geochemistry, Chinese Academy of Sciences, Guangzhou, Guangdong 510640, China

*** ARC Center of Excellence for Core to Crust Fluid Systems (CCFS) and The Institute for Geoscience Research, Department of Applied Geology, Curtin University of Technology, Perth, Western Australia 6845, Australia

$\$$ State Key Laboratory of Geological Processes and Mineral Resources, China University of Geosciences, Wuhan, Hubei 430074, China

${ }^{\dagger}$ Corresponding author: E-mail: lixh@gig.ac.cn; Fax: 86-10-62010846 
remained stable between $c a$. $1.8 \mathrm{Ga}$ and $0.48 \mathrm{Ga}$, despite several episodes of intraplate magmatism (for example, Peng and others, 2005, 2006, 2008, 2011a, 2011b; Yang and others, 2011; Wang and others, 2012a). The eastern part of the NCG was mobilized and modified since the Early Mesozoic, associated with widespread occurrence of magmatism, crustal deformation and lithospheric thinning (for example, Menzies and others, 1993; Yang and others, 2008; Wu and others, 2008 and references therein). Compared with the relatively constrained geological evolution of the eastern NCC, the western NCC, particularly the Alxa Block of the westernmost NCC, is poorly understood (Geng and others, 2002, 2006; Li, ms, 2006; Dong and others, 2007; Dan and others, 2012b), and consequently limits the broader understanding of the NCC.

The westernmost NCC, traditionally named the Alxa Block in Chinese literature (Ren and others, 1987; Wu and others, 1998; Zhai and others, 2000; Geng and others, 2002, 2006), remains the least-studied area in North China. The Alxa Block has long been thought as the westernmost part of the NCC (for example, Ren and others, 1987; Wu and others, 1998; Zhai and others, 2000). Recently, it was considered to be either the western extension of the Yinshan Block (Zhao and others, 2005; Zhao, 2009), or part of the Khondalite Belt (Dong and others, 2007; Geng and others, 2010). However, these models are inconsistent with the newly-identified magmatic rocks in age of $c a .2 .3$ $\mathrm{Ga}$ and $c a$. 0.9 Ga from the eastern Alxa Block (Geng and others, 2002; Dong and others, 2007; Geng and Zhou, 2010; Dan and others, 2012b), as the similar-aged rocks have not been reported within the Yinshan Block and/or the Khondalite Belt. Dan and others (2012b) demonstrate that the eastern Alxa Block has a different thermomagmatic record from the Yinshan Block and Khondalite Belt of the Western Block of the NCC during the Paleoproterozoic time. However, the Neoproterozoic evolution of the Alxa Block remains unclear. The Neoproterozoic granites in the Alxa Block were classified as either S-type (Geng and others, 2002) or A-type granite (Geng and Zhou, 2011). This ambiguity hampers a complete understanding of the Neoproterozoic evolution of the Alxa Block and its relevance to the NCC.

In this study we carried out integrated analyses of in situ zircon U-Pb ages, $\mathrm{Hf}$ and $\mathrm{O}$ isotopes and whole-rock geochemistry for two Neoproterozoic granite plutons, and detrital zircon U-Pb ages for a metasedimentary rock from central Alxa Block. The aim of this work is to (1) place robust chronological and geochemical constrains on the timing and petrogenesis of these Neoproterozoic granites, and (2) investigate the tectonic affinity of the Alxa Block with the NCG and other continents in the Neoproterozoic time.

\section{GEOLOGICAL BACKGROUND}

The North China Craton is the largest and oldest Precambrian craton in China. It has been divided into three Archean continental blocks (that is, the Eastern, Yinshan and Ordos blocks) and three Paleoproterozoic mobile belts (that is, the Khondalite Belt, the Trans-North China Orogen and the Jiao-Liao-Ji Belt) (for example, Zhao and others, 2005) (fig. 1). It is generally considered that the NCC was finally formed during the Late Paleoproterozoic (1900-1850 Ma) (for example, Zhao and others, 2001, 2005; Kusky and Li, 2003; Guo and others, 2005; Kröner and others, 2005, 2006; Kusky, 2011; Zhai and Santosh, 2011). After cratonization the NCC remained stable until the Late Paleozoic, though several intraplate or rift-related magmatic episodes took place between the Late Paleoproterozoic and Paleozoic, including the 1.78 to $1.77 \mathrm{Ga}$ mafic dike swarms (Peng and others, 2005, 2006, 2008), 1.78 to 1.75 Ga rift-related Xiong'er Group volcanism (Zhao and others, 2002; Peng and others, 2005, 2008; He and others, 2009), 1.4 to 1.2 Ga mafic dikes (Zhang and others, 2009; Yang and others, 2011), 925 to $900 \mathrm{Ma}$ mafic dike swarms/sills (Liu and others, 2005; Gao and others, 2009; Peng and others, 2011a, 2011b; Wang and others, 2012a), 860 to $800 \mathrm{Ma}$ acid volcanics (Peng and others, 2010) and 480 Ma kimberlites (Li and others, 2011). 


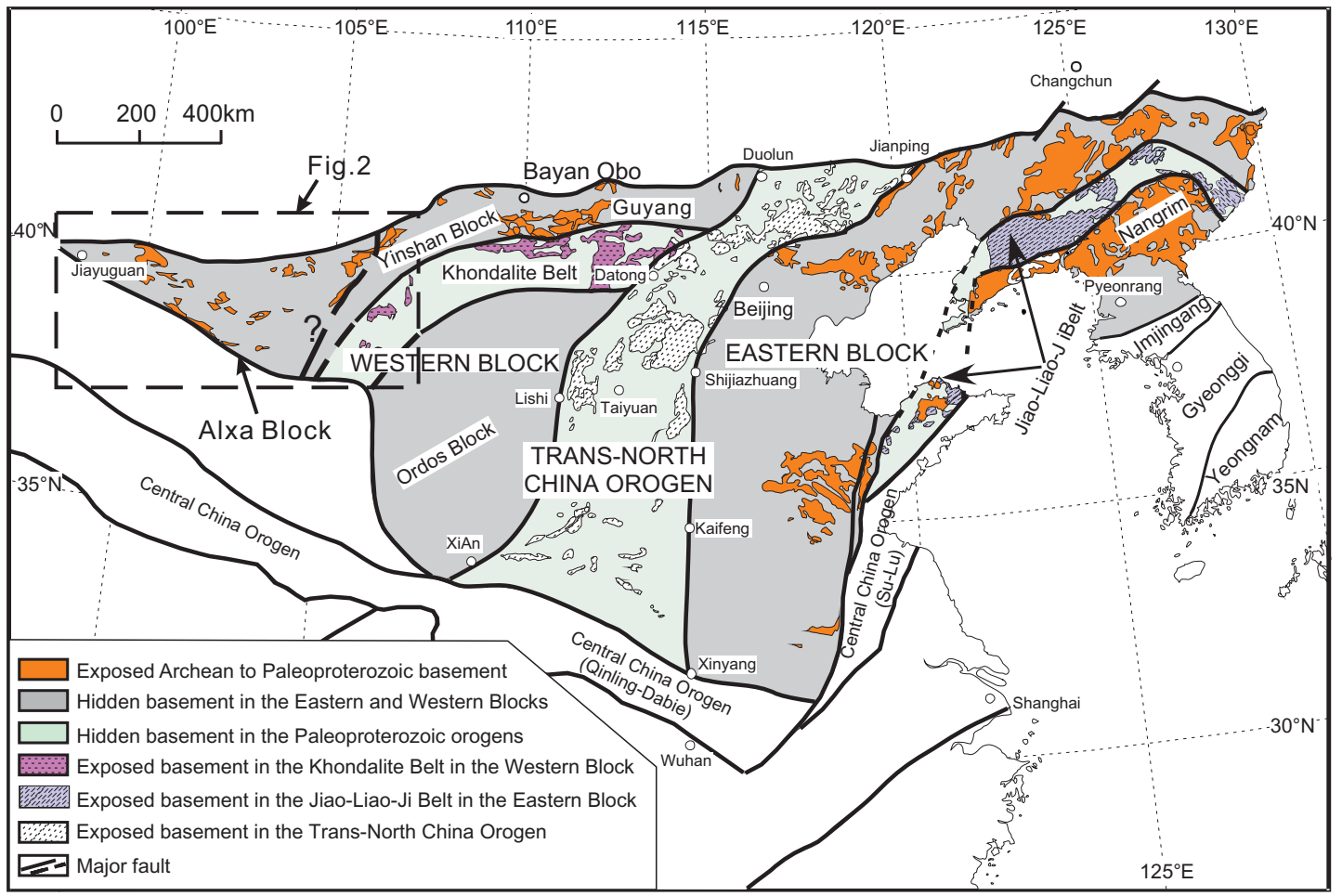

Fig. 1. Tectonic subdivision of the North China Craton (modified from Zhao and others, 2005).

The western part of the NCC consists of the Yinshan and Ordos blocks and the Khondalite Belt (Zhao and others, 2005) (fig. 1). The Alxa Block is generally thought to be the western extension of the Yinshan Block in the westernmost NCC (Zhao and others, 2005). It is largely covered by Cenozoic sediments, and the outcrops of pre-Neoproterozoic crystalline basement rocks are only seen in the southwestern and eastern parts of the block (fig. 2). The oldest rocks, exposed in the poorly dated Beidashan Complex (fig. 2), are $\sim 2.5$ Ga granitic gneisses (Gong and others, 2012). The Longshoushan Complex, exposed in the southwestern Alxa Block, is composed of amphibolite-facies metamorphosed igneous rocks formed at ca. 2.2 to $1.9 \mathrm{Ga}$, and greenschist-facies meta-sedimentary rocks of $<1.72 \mathrm{Ga}$ (Tung and others, 2007; Gong and others, 2011). It was unconformably covered by the Mesoproterozoic to Neoproterozoic sedimentary rocks (GSBGMR, 1989). The Diebusige and Bayanwulashan complexes (fig. 2), exposed in the eastern part of Alxa Block, were previously thought to be the Archean basement rocks in the Alxa Block (Huo and others, 1987; NMBGMR, 1991). Our recent work demonstrated that these complexes were formed during the Early Paleoproterozoic, and underwent two events of metamorphism at $\sim 1.90 \mathrm{Ga}$ and $\sim 1.80 \mathrm{Ga}$ (Dan and others, 2012b). The Mesoproterozoic medium- to low-grade metamorphosed sedimentary sequences were redefined as the Alxa Group (Geng and others, 2007). The Alxa Group was unconformable covered by unmetamorphosed to very low-grade metamorphosed sedimentary sequences (that is, quartzite, slate, dolomite and limestone) (NMBGMR, 1991; Chen and others, 2004), which were probably formed during the Neoproterozoic (Li, ms, 2006).

Geng and others (2002) first recognized Neoproterozoic age (970-845 Ma) granites in three localities within the Alxa Block. Apart from one granitic dike of $30 \mathrm{~m}$ wide located in the Gelintaishan, the remaining two Neoproterozoic granite plutons occurred at the Dabusushan and Naimumaodao (fig. 3). 


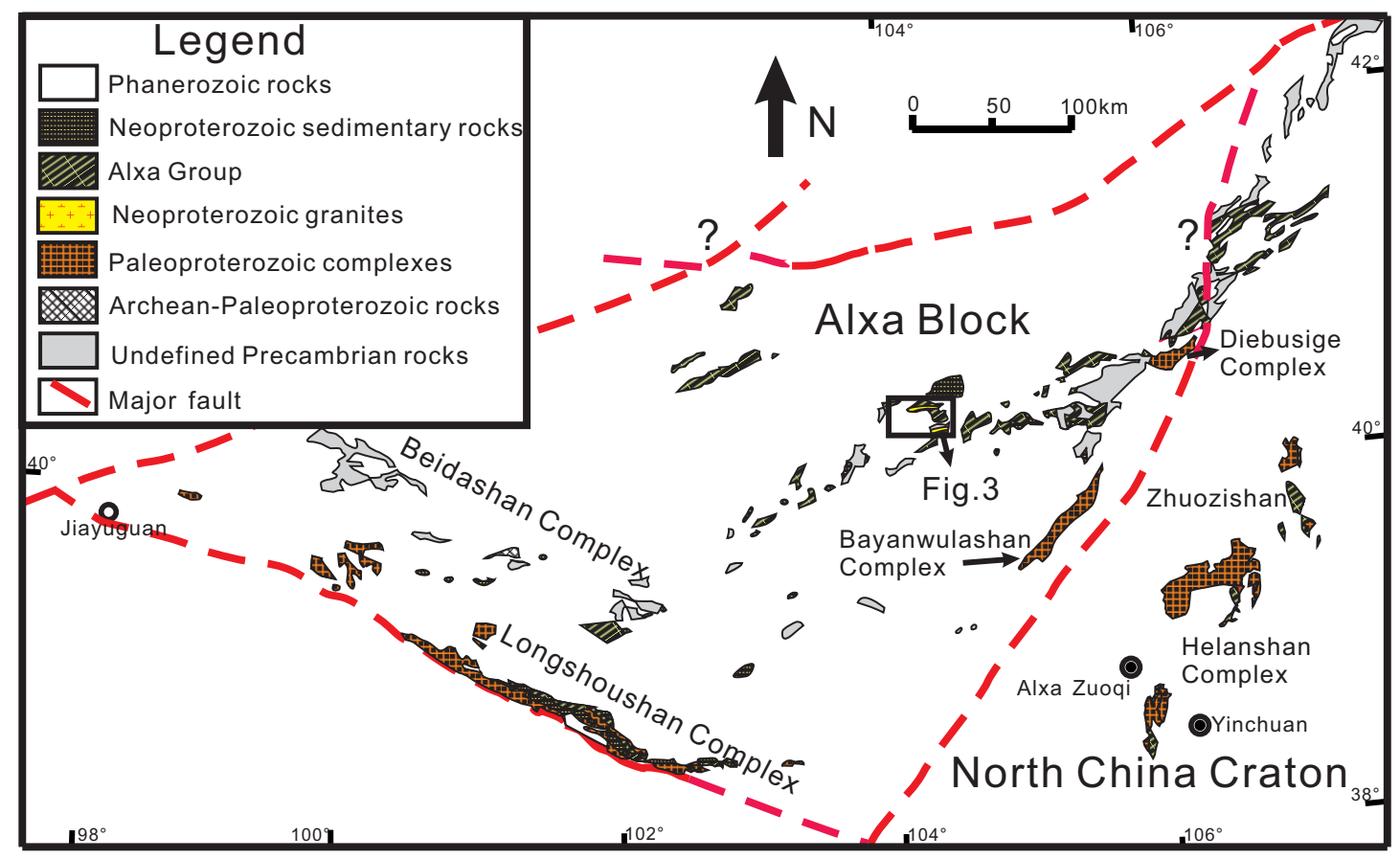

Fig. 2. Distributions of Precambrian basement rocks in the Alxa Block and adjacent areas (modified from Dan and others, 2012b).

FIELD GEOLOGY AND SAMPLE DESCRIPTIONS

The Dabusushan pluton, the largest Neoproterozoic granite pluton in the Alxa Block, crops out over an area of $25 \mathrm{~km}^{2}$. It consists of gneissic two-mica granites containing biotite (2-10 vol.\%), muscovite (5-10 vol.\%), K-feldspar (30-45 vol.\%),

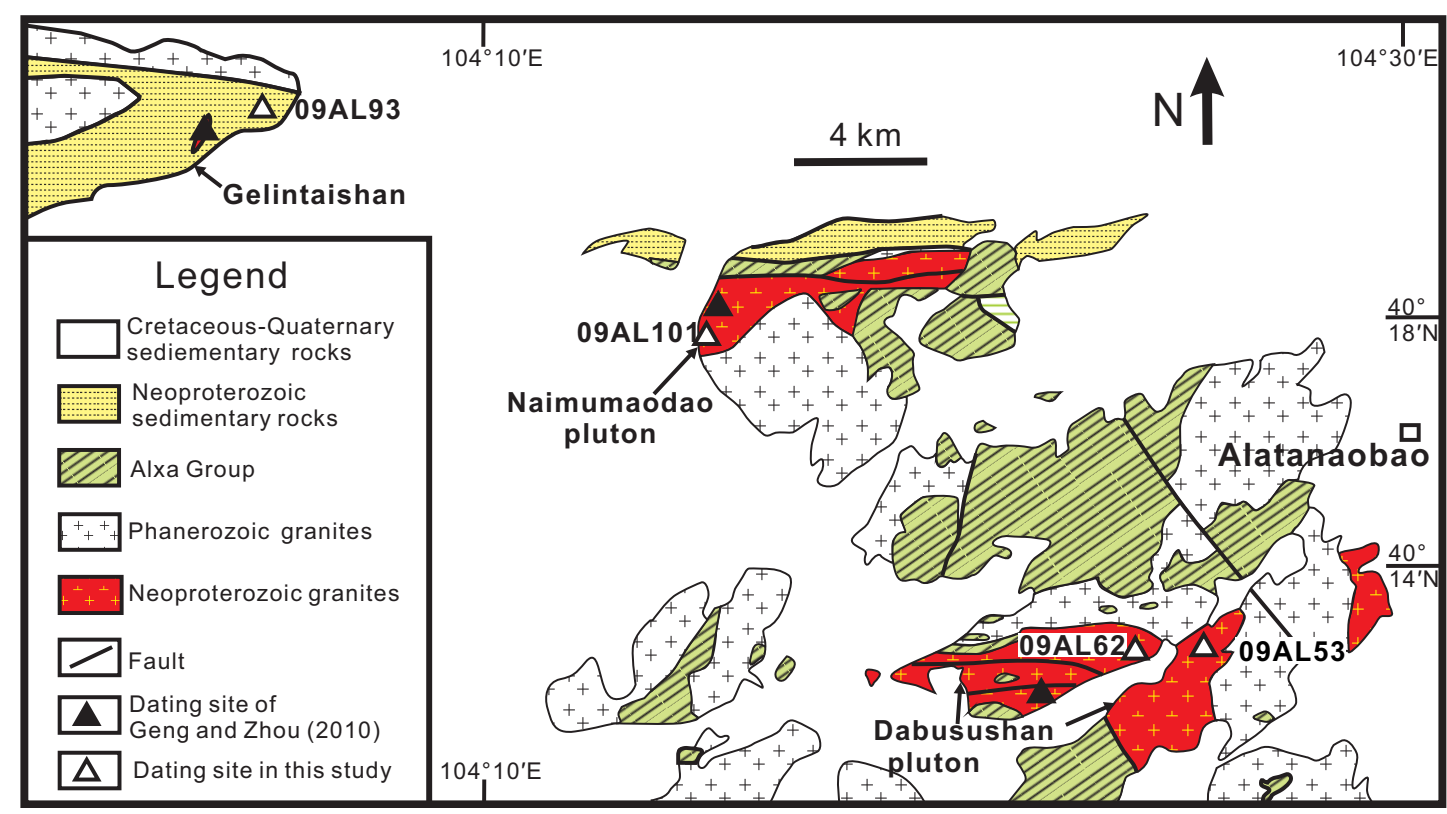

Fig. 3. Geological sketch-map of the Bayinnuoergong area (modified after NMBGMR, 1991), with locations of dating samples. 

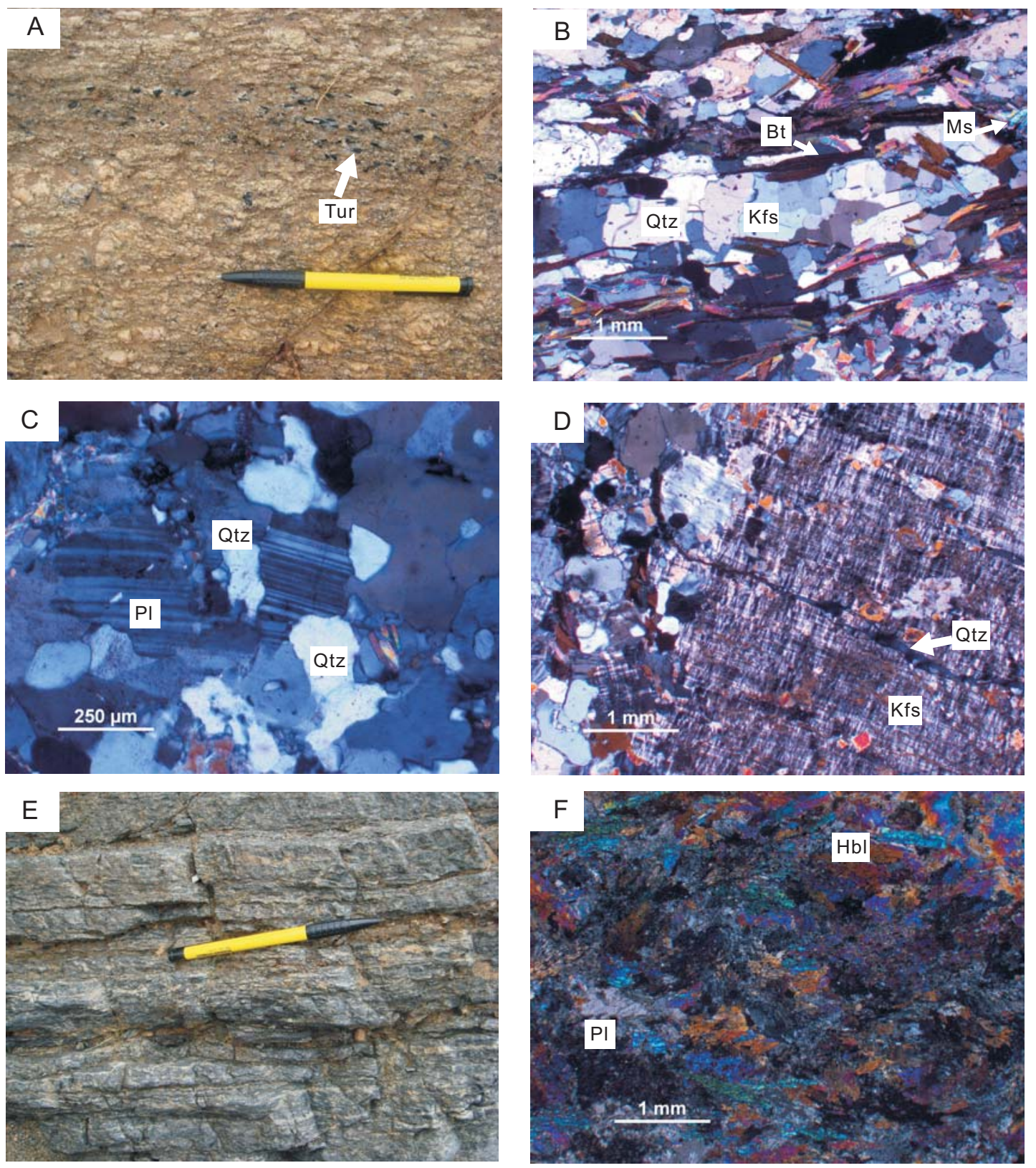

Fig. 4. Field photographs and photomicrographs showing the Neoproterozoic granites and gneisses from the Alxa Block. (A, B) Naimumaodao two-mica granites; (C, D) showing the feldspars were split and filled with quartz; (E, F) Gelintaishan amphibolitic gneisses. The mineral abbreviations are after Kretz (1983).

plagioclase $(10-30 \mathrm{vol} . \%, \mathrm{An}=10-30)$ and quartz $(30-40 \mathrm{vol} . \%)$, as well as trace amount of magnetite, apatite and zircon. The Naimumaodao pluton crops out over an area of 7 $\mathrm{km}^{2}$ to the northwest of the Dabusushan pluton. It also consists of gneissic two-mica granites, containing biotite (2-10 vol.\%), muscovite (2-10 vol.\%), K-feldspar (40-50 vol. $\%)$, plagioclase (10-30 vol.\%, An $=10-30)$ and quartz $(20-30$ vol. $\%)$, as well as trace amounts of tourmaline, magnetite, apatite, zircon and monazite (figs. 4A and 4B). The two-mica granites from the Dabusushan and Naimumaodao plutons were recently dated at $913 \pm 7 \mathrm{Ma}$ and $921 \pm 7 \mathrm{Ma}$, respectively, by LA-ICPMS zircon U-Pb technique (Geng and Zhou, 2010). Both plutons underwent strong deformation as 
seen in the gneissic structure defined by parallel-oriented mica and augen feldspar crystals. Under microscope, a few feldspars were split and filled with quartz (figs. 4C and 4D). Two samples (09AL53 and 09AL62) from the Dabusushan pluton and one (09AL101) from the Naimumaodao pluton were selected for in situ zircon U-Pb dating and Hf-O isotopic analyses. The sample sites are shown in figure 3.

In addition, a gneissic biotite granitic dike intruded the Deerhetongte Formation of Alxa Group at Gelintaishan (fig. 3) was dated at $904 \pm 7$ Ma by LA-ICPMS zircon U-Pb technique (Geng and Zhou, 2010). We collected an amphibolitic gneiss sample (09AL93) from the Deerhetongte Formation (fig. 3) for in situ zircon U-Pb dating and $\mathrm{Hf}-\mathrm{O}$ isotopic analysis. The amphibolitic gneiss is interfingered with mica-quartz schist, and it underwent strong deformation and metamorphism to amphibolite facies (figs. $4 \mathrm{E}$ and $4 \mathrm{~F}$ ). Geochemical characteristics suggest that the amphibolitic gneiss was of metasedimentary origin (see below).

\section{ANALYTICAL PROCEDURES}

\section{Whole-rock Geochemistry}

Major element oxides were analyzed on fused glass beads using a Rigaku RIX 2000 X-ray fluorescence spectrometer at the Guangzhou Institute of Geochemistry, Chinese Academy of Sciences. Calibration lines used in quantification were produced by bivariate regression of data from 36 reference materials encompassing a wide range of silicate compositions ( $\mathrm{Li}$ and others, 2005). Analytical uncertainties are between 1 percent and 5 percent. Trace elements were analyzed using an Agilent 7500a ICP-MS at the Institute of Geology and Geophysics, Chinese Academy of Sciences (IGG-CAS), Beijing. Analytical procedures were similar to those described by Li and others (2000). About $50 \mathrm{mg}$ of each powdered sample were dissolved in a high-pressure Teflon bomb for 48 hours using a $\mathrm{HF}+\mathrm{HNO}_{3}$ mixture. An internal standard solution containing the single element Rh was used to monitor signal drift during counting. A set of USGS and Chinese national rock standards, including BHVO-2, BIR-1, AGV-2, RGM-2, GSP-2, JG-1 and JG-2 were chosen for calibration. Analytical precision typically is better than 5 percent. Geochemical results are presented in table 1.

$\mathrm{Nd}$ isotopic compositions were determined using a Micromass Isoprobe multicollector ICP-MS at IGG-CAS, and analytical procedures were similar to those described by Li and others (2004). Nd fractions were separated by passing through cation columns followed by HDEHP columns. Measured ${ }^{143} \mathrm{Nd} /{ }^{144} \mathrm{Nd}$ ratios were normalized to ${ }^{146} \mathrm{Nd} /{ }^{144} \mathrm{Nd}=0.7219$, and further adjusted relative to the Shin Etsu JNdi-1 standard of 0.512115 . Whole-rock Nd isotopic data are listed in table 2.

\section{Zircon $U-P b$ Dating}

Zircon concentrates were separated from approximately 2-kg rock samples by conventional magnetic and density techniques to concentrate non-magnetic, heavy fractions. Zircon grains, together with zircon standards 91500, Plešovice, and Penglai were mounted in epoxy resin mounts, which were then polished to section the crystals in half for analysis. All zircons were documented with transmitted and reflected light photomicrographs, as well as cathodoluminescence (CL) images, to reveal their internal structures.

Measurements of $\mathrm{U}, \mathrm{Th}$ and $\mathrm{Pb}$ were conducted using the Cameca IMS-1280 at IGG-CAS. U-Th-Pb ratios were determined relative to the standard zircon Plešovice (Sláma and others, 2008), and their absolute abundances were calibrated to the standard zircon 91500 (Wiedenbeck and others, 1995), using operating and data processing procedures similar to those described by Li and others (2009a). The $\mathrm{O}_{2}{ }^{-}$ primary ion beam was accelerated at $13 \mathrm{kV}$, with an intensity of $c a .8 \mathrm{nA}$. The ellipsoidal spot is about $20 \times 30 \mu \mathrm{m}$ in size. Positive secondary ions were extracted with a $10 \mathrm{kV}$ 
TABLE 1

Geochemical data for the Neoproterozoic rocks from Alxa Block

\begin{tabular}{|c|c|c|c|c|c|c|c|c|}
\hline \multicolumn{3}{|c|}{ Rock type } & \multicolumn{4}{|c|}{ Dabusushan two-mica granite } & \multirow[b]{2}{*}{ 09AL65-1 } & \multirow[b]{2}{*}{ 09AL65-2 } \\
\hline Sample & .09AL53 & 09AL54 & 09AL55 & 09AL62 & 09AL63 & 09AL64 & & \\
\hline \multicolumn{9}{|c|}{ Major elements (wt.\%) } \\
\hline $\mathrm{SiO}_{2}$ & 79.06 & 75.99 & 73.88 & 74.42 & 74.66 & 74.44 & 75.83 & 75.53 \\
\hline $\mathrm{TiO}_{2}$ & 0.18 & 0.15 & 0.15 & 0.28 & 0.22 & 0.25 & 0.08 & 0.09 \\
\hline $\mathrm{Al}_{2} \mathrm{O}_{3}$ & 11.59 & 13.24 & 14.13 & 13.62 & 14.19 & 13.86 & 14.03 & 14.15 \\
\hline $\mathrm{Fe}_{2} \mathrm{O}_{3}^{\mathrm{T}}$ & 1.37 & 1.10 & 1.00 & 2.18 & 1.52 & 2.18 & 0.91 & 1.07 \\
\hline $\mathrm{MnO}$ & 0.01 & 0.01 & 0.01 & 0.02 & 0.01 & 0.02 & 0.01 & 0.01 \\
\hline $\mathrm{MgO}$ & 0.21 & 0.19 & 0.19 & 0.32 & 0.30 & 0.32 & 0.14 & 0.14 \\
\hline $\mathrm{CaO}$ & 1.08 & 0.89 & 0.63 & 1.32 & 1.26 & 1.21 & 0.63 & 0.64 \\
\hline $\mathrm{Na}_{2} \mathrm{O}$ & 2.13 & 2.19 & 2.06 & 2.76 & 2.56 & 2.29 & 2.90 & 2.81 \\
\hline $\mathrm{K}_{2} \mathrm{O}$ & 4.32 & 6.21 & 7.95 & 5.04 & 5.21 & 5.28 & 5.44 & 5.52 \\
\hline $\mathrm{P}_{2} \mathrm{O}_{5}$ & 0.09 & 0.09 & 0.10 & 0.09 & 0.15 & 0.18 & 0.17 & 0.18 \\
\hline Total & 100.04 & 100.09 & 100.11 & 100.06 & 100.08 & 100.03 & 100.15 & 100.14 \\
\hline L.O.I & 0.86 & 0.74 & 0.72 & 0.44 & 0.90 & 1.01 & 0.82 & 0.90 \\
\hline \multicolumn{9}{|l|}{ DF3 } \\
\hline $\mathrm{C}$ & 1.7 & 1.5 & 1.2 & 1.4 & 2.4 & 2.6 & 2.6 & 2.8 \\
\hline $\mathrm{A} / \mathrm{CNK}$ & 1.14 & 1.11 & 1.08 & 1.10 & 1.17 & 1.19 & 1.19 & 1.20 \\
\hline \multicolumn{9}{|c|}{ Trace element (ppm) } \\
\hline $\mathrm{Sc}$ & 0.31 & 0.21 & 0.25 & 5.0 & 1.4 & 1.9 & 0.60 & 1.1 \\
\hline V & 4.0 & 3.6 & 6.1 & 10 & 8.6 & 8.3 & 0.74 & 0.70 \\
\hline $\mathrm{Cr}$ & 1.4 & 1.9 & 1.3 & 2.1 & 3.8 & 3.1 & 0.53 & 0.56 \\
\hline $\mathrm{Ni}$ & 1.3 & 1.1 & 0.65 & 1.4 & 2.6 & 2.6 & 0.33 & 0.36 \\
\hline $\mathrm{Ga}$ & 19 & 18 & 18 & 20 & 21 & 24 & 22 & 23 \\
\hline $\mathrm{Rb}$ & 190 & 257 & 295 & 236 & 204 & 222 & 417 & 420 \\
\hline $\mathrm{Sr}$ & 50 & 63 & 66 & 72 & 114 & 68 & 21 & 21 \\
\hline Y & 13 & 15 & 19 & 51 & 14 & 27 & 15 & 15 \\
\hline $\mathrm{Zr}$ & 109 & 104 & 118 & 180 & 162 & 134 & 45 & 41 \\
\hline $\mathrm{Nb}$ & 17 & 15 & 15 & 17 & 14 & 17 & 11 & 12 \\
\hline $\mathrm{Ba}$ & 195 & 312 & 368 & 549 & 911 & 320 & 27 & 30 \\
\hline $\mathrm{La}$ & 25 & 27 & 26 & 55 & 40 & 45 & 9.0 & 8.2 \\
\hline $\mathrm{Ce}$ & 59 & 62 & 60 & 108 & 86 & 97 & 23 & 21 \\
\hline $\operatorname{Pr}$ & 6.9 & 6.9 & 6.4 & 12 & 8.9 & 11 & 2.7 & 2.3 \\
\hline $\mathrm{Nd}$ & 28 & 26 & 24 & 43 & 32 & 40 & 10 & 9.1 \\
\hline $\mathrm{Sm}$ & 7.7 & 7.1 & 6.7 & 8.6 & 7.0 & 9.9 & 3.6 & 3.2 \\
\hline $\mathrm{Eu}$ & 0.42 & 0.54 & 0.62 & 0.68 & 1.1 & 0.60 & 0.11 & 0.10 \\
\hline $\mathrm{Gd}$ & 6.0 & 5.4 & 5.2 & 8.9 & 6.1 & 8.2 & 2.7 & 2.2 \\
\hline $\mathrm{Tb}$ & 1.0 & 1.0 & 1.0 & 1.5 & 0.91 & 1.4 & 0.59 & 0.53 \\
\hline Dy & 4.1 & 4.1 & 4.2 & 7.6 & 3.7 & 6.0 & 3.0 & 2.7 \\
\hline Ho & 0.39 & 0.47 & 0.55 & 1.5 & 0.44 & 0.91 & 0.50 & 0.47 \\
\hline Er & 1.2 & 1.2 & 1.3 & 3.8 & 1.4 & 2.4 & 1.1 & 1.0 \\
\hline $\mathrm{Tm}$ & 0.07 & 0.08 & 0.11 & 0.61 & 0.14 & 0.28 & 0.16 & 0.16 \\
\hline $\mathrm{Yb}$ & 0.78 & 0.79 & 0.87 & 3.7 & 1.0 & 1.9 & 1.1 & 1.0 \\
\hline $\mathrm{Lu}$ & 0.11 & 0.11 & 0.12 & 0.56 & 0.14 & 0.28 & 0.17 & 0.16 \\
\hline $\mathrm{Hf}$ & 3.7 & 3.4 & 3.6 & 5.2 & 4.6 & 4.7 & 2.1 & 1.9 \\
\hline $\mathrm{Ta}$ & 1.6 & 0.70 & 0.70 & 1.0 & 0.78 & 1.3 & 1.0 & 1.8 \\
\hline Th & 17 & 16 & 14 & 29 & 15 & 22 & 8.2 & 8.6 \\
\hline $\mathrm{U}$ & 2.9 & 3.0 & 3.8 & 4.0 & 2.5 & 3.2 & 1.4 & 1.4 \\
\hline
\end{tabular}


TABLE 1

(continued)

\begin{tabular}{|c|c|c|c|c|c|c|c|c|}
\hline \multirow[t]{2}{*}{ Rock type } & \multicolumn{3}{|c|}{$\begin{array}{c}\text { Dabusushan } \\
\text { two-mica granite }\end{array}$} & \multicolumn{3}{|c|}{$\begin{array}{c}\text { Naimumaodao } \\
\text { two-mica granite }\end{array}$} & \multicolumn{2}{|c|}{$\begin{array}{c}\text { Gelintaishan } \\
\text { Amphibolitic gneiss }\end{array}$} \\
\hline & 09AL53 & 09AL54 & 09AL55 & 09AL62 & 09AL63 & 09AL64 & 09AL65-1 & 09AL65-2 \\
\hline \multicolumn{9}{|c|}{ Major elements (wt.\%) } \\
\hline $\mathrm{SiO}_{2}$ & 76.03 & 75.76 & 74.97 & 76.38 & 77.39 & 80.04 & 52.45 & 48.83 \\
\hline $\mathrm{TiO}_{2}$ & 0.06 & 2.40 & 0.07 & 0.33 & 0.40 & 0.26 & 0.54 & 0.85 \\
\hline $\mathrm{Al}_{2} \mathrm{O}_{3}$ & 15.90 & 12.14 & 13.64 & 13.92 & 13.23 & 11.30 & 7.73 & 14.44 \\
\hline $\mathrm{Fe}_{2} \mathrm{O}_{3}^{\mathrm{T}}$ & 0.21 & 0.84 & 0.70 & 2.10 & 1.50 & 2.02 & 4.06 & 4.90 \\
\hline $\mathrm{MnO}$ & 0.01 & 0.01 & 0.01 & 0.01 & 0.01 & 0.04 & 0.08 & 0.08 \\
\hline $\mathrm{MgO}$ & 0.19 & 0.33 & 0.18 & 0.92 & 1.03 & 1.16 & 12.95 & 13.25 \\
\hline $\mathrm{CaO}$ & 1.19 & 2.12 & 1.80 & 0.55 & 0.87 & 0.43 & 19.03 & 13.53 \\
\hline $\mathrm{Na}_{2} \mathrm{O}$ & 4.61 & 4.83 & 2.14 & 3.05 & 4.51 & 3.20 & 0.37 & 0.39 \\
\hline $\mathrm{K}_{2} \mathrm{O}$ & 2.02 & 0.89 & 6.35 & 2.69 & 1.08 & 1.51 & 2.18 & 3.12 \\
\hline $\mathrm{P}_{2} \mathrm{O}_{5}$ & 0.02 & 0.86 & 0.19 & 0.13 & 0.14 & 0.10 & 0.08 & 0.14 \\
\hline Total & 100.24 & 100.19 & 100.06 & 100.08 & 100.17 & 100.06 & 99.47 & 99.54 \\
\hline L.O.I & 0.85 & 0.59 & 1.83 & 1.54 & 1.22 & 1.41 & 3.57 & 4.58 \\
\hline DF3 & & & & & & & -2.37 & -4.67 \\
\hline $\mathrm{C}$ & 4.0 & 1.4 & 0.4 & 5.3 & 3.4 & 3.9 & & \\
\hline $\mathrm{A} / \mathrm{CNK}$ & 1.33 & 0.95 & 1.00 & 1.56 & 1.30 & 1.47 & 0.21 & 0.50 \\
\hline \multicolumn{9}{|c|}{ Trace element (ppm) } \\
\hline $\mathrm{Sc}$ & 0.66 & 33 & 1.8 & 8.4 & 7.9 & 6.9 & 10 & 15 \\
\hline V & 1.5 & 74 & 4.0 & 24 & 21 & 18 & 116 & 158 \\
\hline $\mathrm{Cr}$ & 0.66 & 80 & 1.5 & 9.2 & 14 & 8.0 & 41 & 67 \\
\hline $\mathrm{Ni}$ & 0.88 & 4.0 & 2.5 & 2.0 & 4.0 & 2.3 & 14 & 21 \\
\hline $\mathrm{Ga}$ & 21 & 14 & 20 & 18 & 17 & 15 & 10 & 20 \\
\hline $\mathrm{Rb}$ & 59 & 47 & 244 & 134 & 49 & 76 & 122 & 169 \\
\hline $\mathrm{Sr}$ & 418 & 50 & 103 & 35 & 117 & 52 & 89 & 138 \\
\hline $\mathrm{Y}$ & 7.2 & 117 & 18 & 35 & 37 & 28 & 27 & 34 \\
\hline $\mathrm{Zr}$ & 31 & 1012 & 41 & 126 & 163 & 92 & 193 & 181 \\
\hline $\mathrm{Nb}$ & 4.2 & 73 & 7.5 & 11 & 13 & 8.8 & 9.8 & 15 \\
\hline $\mathrm{Ba}$ & 1125 & 97 & 234 & 253 & 182 & 149 & 222 & 337 \\
\hline $\mathrm{La}$ & 8.5 & 135 & 10 & 35 & 34 & 25 & 28 & 35 \\
\hline $\mathrm{Ce}$ & 16 & 272 & 22 & 74 & 78 & 55 & 54 & 72 \\
\hline $\operatorname{Pr}$ & 1.8 & 33 & 2.6 & 8.2 & 8.2 & 6.0 & 6.0 & 8.3 \\
\hline $\mathrm{Nd}$ & 7.2 & 127 & 9.8 & 31 & 32 & 24 & 24 & 33 \\
\hline $\mathrm{Sm}$ & 1.6 & 28 & 3.1 & 6.7 & 7.0 & 5.4 & 5.1 & 6.7 \\
\hline $\mathrm{Eu}$ & 0.76 & 2.2 & 0.41 & 0.61 & 0.57 & 0.46 & 0.86 & 1.2 \\
\hline $\mathrm{Gd}$ & 1.5 & 26 & 2.2 & 6.2 & 6.6 & 5.0 & 4.4 & 5.5 \\
\hline $\mathrm{Tb}$ & 0.25 & 4.5 & 0.54 & 1.1 & 1.2 & 0.91 & 0.72 & 0.91 \\
\hline Dy & 1.2 & 22 & 2.9 & 5.7 & 6.1 & 4.8 & 4.2 & 5.3 \\
\hline Ho & 0.22 & 3.8 & 0.59 & 1.1 & 1.2 & 0.93 & 0.75 & 1.0 \\
\hline $\mathrm{Er}$ & 0.60 & 10 & 1.2 & 2.8 & 3.0 & 2.3 & 2.1 & 2.8 \\
\hline $\mathrm{Tm}$ & 0.09 & 1.4 & 0.21 & 0.46 & 0.49 & 0.38 & 0.35 & 0.46 \\
\hline $\mathrm{Yb}$ & 0.59 & 8.9 & 1.3 & 2.7 & 3.0 & 2.3 & 2.2 & 2.8 \\
\hline $\mathrm{Lu}$ & 0.09 & 1.3 & 0.21 & 0.41 & 0.45 & 0.33 & 0.35 & 0.44 \\
\hline $\mathrm{Hf}$ & 1.4 & 29 & 2.0 & 4.0 & 4.6 & 3.0 & 5.2 & 5.1 \\
\hline $\mathrm{Ta}$ & 0.30 & 2.9 & 0.83 & 1.0 & 1.1 & 1.1 & 0.93 & 1.3 \\
\hline $\mathrm{Th}$ & 2.4 & 61 & 8.1 & 17 & 19 & 14 & 8.7 & 14 \\
\hline $\mathrm{U}$ & 1.2 & 5.4 & 2.6 & 1.4 & 2.9 & 2.4 & 1.9 & 2.6 \\
\hline
\end{tabular}

$\mathrm{DF} 3=-0.21 \mathrm{SiO}_{2}-0.32 \mathrm{Fe}_{2} \mathrm{O}_{3}{ }^{\mathrm{T}}-0.98 \mathrm{MgO}+0.55 \mathrm{CaO}+1.46 \mathrm{Na}_{2} \mathrm{O}+0.54 \mathrm{~K}_{2} \mathrm{O}+10.44$ (Shaw, 1972). $\mathrm{A} / \mathrm{CNK}=$ molar $\mathrm{Al}_{2} \mathrm{O}_{3} /\left(\mathrm{CaO}+\mathrm{Na}_{2} \mathrm{O}+\mathrm{K}_{2} \mathrm{O}\right)$.

$\mathrm{C}=$ normative corundum. 
TABLE 2

Whole rock Sm-Nd data for the Neoproterozoic granites from Alxa Block

\begin{tabular}{|c|c|c|c|c|c|c|c|}
\hline Sample & $\frac{{ }^{147} \mathrm{Sm}}{{ }^{144} \mathrm{Nd}}$ & $\frac{{ }^{143} \mathrm{Nd}}{{ }^{144} \mathrm{Nd}}$ & $2 \sigma_{\mathrm{m}}$ & $\varepsilon_{\mathrm{Nd}}(\mathrm{t})$ & $\mathrm{T}_{\mathrm{DM}}(\mathrm{Ga})$ & $\mathrm{T}_{2 \mathrm{DM}}(\mathrm{Ga})$ & $\mathrm{f}_{\mathrm{Sm} / \mathrm{Nd}}$ \\
\hline \multicolumn{8}{|c|}{ Ca. 0.91 Ga Dabusushan two-mica granite } \\
\hline 09AL53 & 0.1662 & 0.512177 & 0.000007 & -5.5 & 3.11 & 2.00 & -0.16 \\
\hline 09AL54 & 0.1650 & 0.512217 & 0.000012 & -4.5 & 2.92 & 1.93 & -0.16 \\
\hline 09AL55 & 0.1687 & 0.512181 & 0.000013 & -5.7 & 3.27 & 2.02 & -0.14 \\
\hline 09AL66 & 0.1343 & 0.511747 & 0.000007 & -10.1 & 2.68 & 2.38 & -0.32 \\
\hline 09AL62 & 0.1208 & 0.511747 & 0.000010 & -8.6 & 2.30 & 2.26 & -0.39 \\
\hline 09AL63 & 0.1322 & 0.511883 & 0.000009 & -7.2 & 2.36 & 2.15 & -0.33 \\
\hline 09AL64 & 0.1495 & 0.511993 & 0.000009 & -7.1 & 2.74 & 2.14 & -0.24 \\
\hline \multicolumn{8}{|c|}{ Ca. 0.93 Ga Naimumaodao two-mica granite } \\
\hline 09AL101 & 0.1265 & 0.511938 & 0.000012 & -5.3 & 2.12 & 2.01 & -0.36 \\
\hline 09AL105 & 0.1359 & 0.511971 & 0.000012 & -5.8 & 2.31 & 2.05 & -0.31 \\
\hline \multicolumn{8}{|c|}{ Standard reference } \\
\hline
\end{tabular}

$\varepsilon_{\mathrm{Nd}}(\mathrm{t})=10000 \times\left\{\left[\left({ }^{143} \mathrm{Nd} /{ }^{144} \mathrm{Nd}\right)_{\mathrm{s}}-\left({ }^{147} \mathrm{Sm} /{ }^{144} \mathrm{Nd}\right)_{\mathrm{s}} \times\left(\mathrm{e}^{\lambda \mathrm{t}}-1\right)\right] /\left[\left({ }^{143} \mathrm{Nd} /{ }^{144} \mathrm{Nd}\right)_{\mathrm{CHUR}, 0}-\left({ }^{147} \mathrm{Sm} /\right.\right.\right.$ $\left.\left.\left.{ }^{144} \mathrm{Nd}\right)_{\mathrm{CHUR}} \times\left(\mathrm{e}^{\lambda \mathrm{t}}-1\right)\right]-1\right\} ; \mathrm{T}_{\mathrm{DM}}=1 / \lambda \times \ln \left\{1+\left[\left({ }^{143} \mathrm{Nd} /{ }^{144} \mathrm{Nd}\right)_{\mathrm{s}}-\left({ }^{143} \mathrm{Nd} /{ }^{144} \mathrm{Nd}\right)_{\mathrm{DM}}\right] /\left[\left({ }^{147} \mathrm{Sm} /\right.\right.\right.$ $\left.\left.\left.{ }^{144} \mathrm{Nd}\right)_{\mathrm{s}}-\left({ }^{147} \mathrm{Sm} /{ }^{144} \mathrm{Nd}\right)_{\mathrm{DM}}\right]\right\} ; \mathrm{T}_{2 \mathrm{DM}}=\mathrm{T}_{\mathrm{DM}}-\left(\mathrm{T}_{\mathrm{DM}}-\mathrm{t}\right)\left(\left(\mathrm{f}_{\mathrm{c}}-\mathrm{f}_{\mathrm{s}}\right) /\left(\mathrm{f}_{\mathrm{c}}-\mathrm{f}_{\mathrm{DM}}\right)\right) ; \mathrm{f}_{\mathrm{Sm} / \mathrm{Nd}}=\left({ }^{14}{ }^{\mathrm{Sm}} /{ }^{144} \mathrm{Nd}\right)_{\mathrm{s}} /$ $\left({ }^{147} \mathrm{Sm} /{ }^{144} \mathrm{Nd}\right)_{\text {CHUR }}-1$; where $\mathrm{f}_{\mathrm{c}}, \mathrm{f}_{\mathrm{s}}$ and $\mathrm{f}_{\mathrm{DM}}$ are the $\mathrm{f}_{\mathrm{Sm} / \mathrm{Nd}}$ values of the continental crust, sample and the depleted mantle; $\mathrm{f}_{\mathrm{c}}=-0.4, \mathrm{f}_{\mathrm{DM}}=0.08592 ; \mathrm{t}=$ crystallization time; $\left({ }^{147} \mathrm{Sm} /{ }^{144} \mathrm{Nd}\right)_{\mathrm{s}}$ and $\left({ }^{143} \mathrm{Nd} /\right.$ $\left.{ }^{144} \mathrm{Nd}\right)_{\mathrm{s}}$ are values of analysed sample; $\left({ }^{147} \mathrm{Sm} /{ }^{144} \mathrm{Nd}\right)_{\mathrm{CHUR}}=0.1967$ and $\left({ }^{143} \mathrm{Nd} /{ }^{144} \mathrm{Nd}\right)_{\mathrm{CHUR}, 0}=0.512638$; $\left({ }^{147} \mathrm{Sm} /{ }^{144} \mathrm{Nd}\right)_{\mathrm{DM}}=0.2135$ and $\left({ }^{143} \mathrm{Nd} /{ }^{144} \mathrm{Nd}\right)_{\mathrm{DM}}=0.51315 ;\left({ }^{147} \mathrm{Sm} /{ }^{144} \mathrm{Nd}\right)_{\mathrm{c}}=0.118$.

potential. A mass resolution of $c a .5400$ was used to separate $\mathrm{Pb}^{+}$peaks from isobaric interferences. A single electron multiplier was used in ion-counting mode to measure secondary ion beam intensities by peak jumping. Each measurement consists of 7 cycles. Analyses of the Plešovice standard were interspersed with those of unknown grains. A long-term uncertainty of 1.5 percent (1 RSD) for ${ }^{206} \mathrm{~Pb} /{ }^{238} \mathrm{U}$ measurements of the standard zircons was propagated to the unknowns ( $\mathrm{Li}$ and others, 2010a), despite the measured ${ }^{206} \mathrm{~Pb} /{ }^{238} \mathrm{U}$ error during the course of this study generally is around 1 percent ( $1 \mathrm{RSD}$ ) or less. Measured compositions were corrected for common $\mathrm{Pb}$ using non-radiogenic ${ }^{204} \mathrm{~Pb}$. Corrections were sufficiently small to be insensitive to the choice of common $\mathrm{Pb}$ composition, and an average of present-day crustal composition (Stacey and Kramers, 1975) was used for the common Pb, assuming that the common $\mathrm{Pb}$ was largely surface contamination introduced during sample preparation. Uncertainties on individual analyses in the data tables are reported at a $1 \sigma$ level. Mean ages for pooled $\mathrm{U} / \mathrm{Pb}$ and $\mathrm{Pb} / \mathrm{Pb}$ analyses are quoted with $2 \sigma$ and/or 95 percent confidence intervals. The weighted mean $\mathrm{U}-\mathrm{Pb}$ ages and Concordia plots were processed using Isoplot/Ex v.3.0 program (Ludwig, 2003). SIMS zircon U-Pb isotopic data are presented in table 3 .

The LA-ICPMS U-Pb dating for detritus zircons from the amphibolitic gneiss (sample 09AL93) was conducted using an Agilent 7500a ICP-MS with an attached 193 nm excimer ArF laser-ablation system (GeoLas Plus) at IGG-CAS. The analytical procedures are similar to those described by Xie and others (2008). Before analysis, the sample surface was cleaned with ethanol to eliminate possible contamination. The parameters were $32 \mu \mathrm{m}$ spot size and $8 \mathrm{~Hz}$ repetition rate in this study. Every 10 unknown analyses were followed by measurements of two zircon 91500, two GJ-1 and one NIST SRM 610 standards. ${ }^{207} \mathrm{~Pb} /{ }^{206} \mathrm{~Pb}$ and ${ }^{206} \mathrm{~Pb} /{ }^{238} \mathrm{U}$ ratios were calculated using the ICPMSDataCal software (Liu and others, 2010a, 2010b), using the zircon standard 91500 as an external standard. Common $\mathrm{Pb}$ was corrected according to the method 
Westernmost North China and tectonic implications

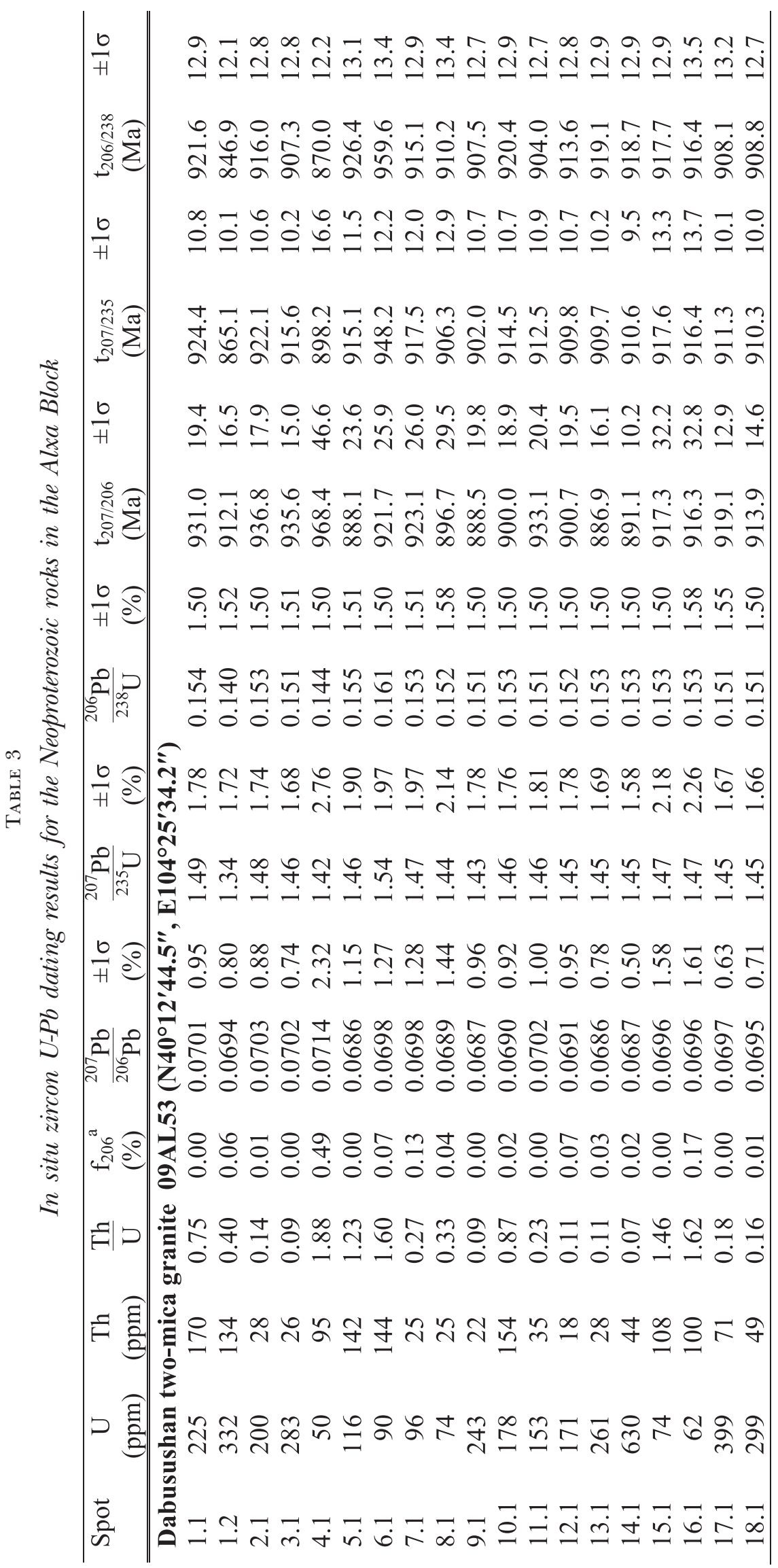




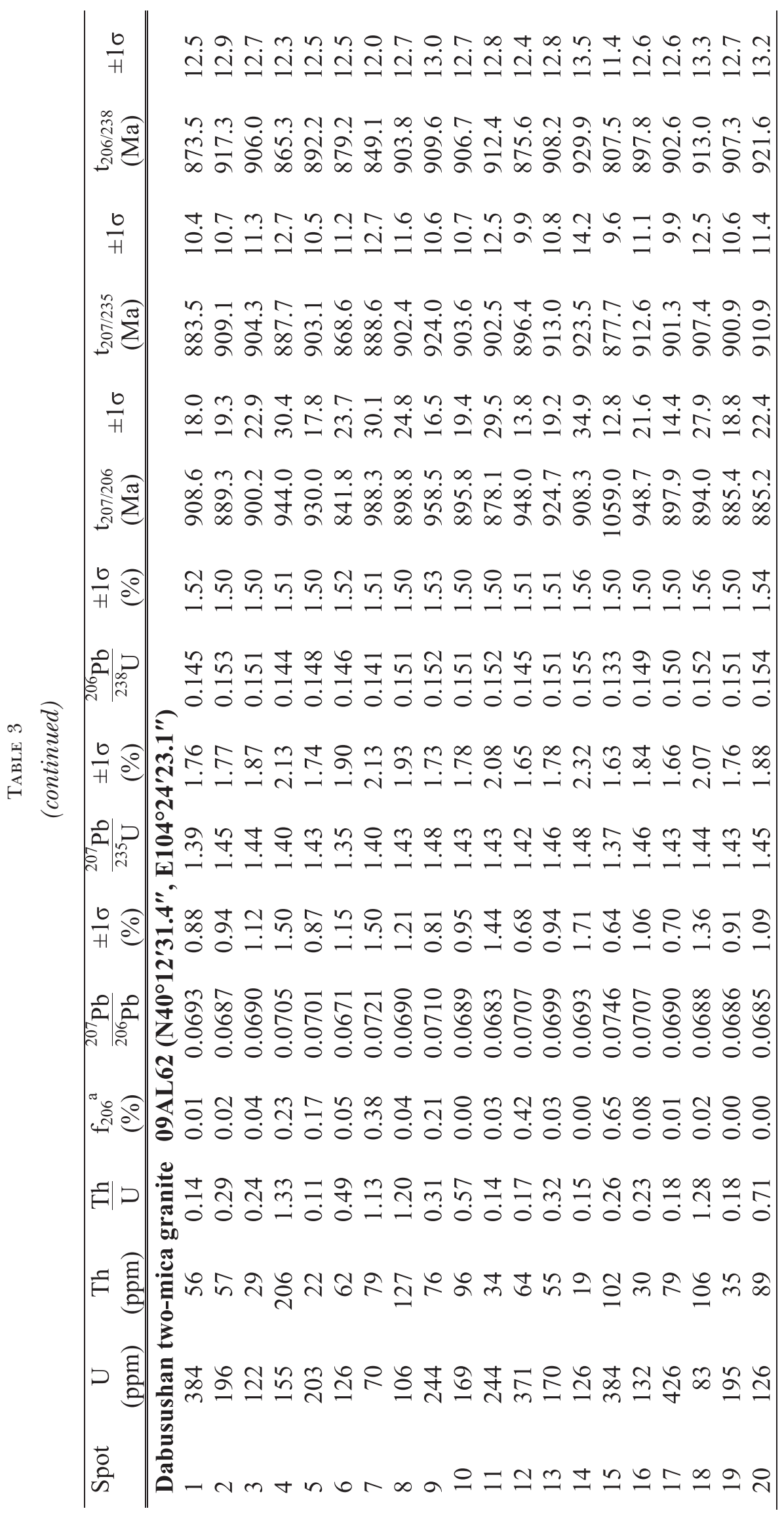


Westernmost North China and tectonic implications

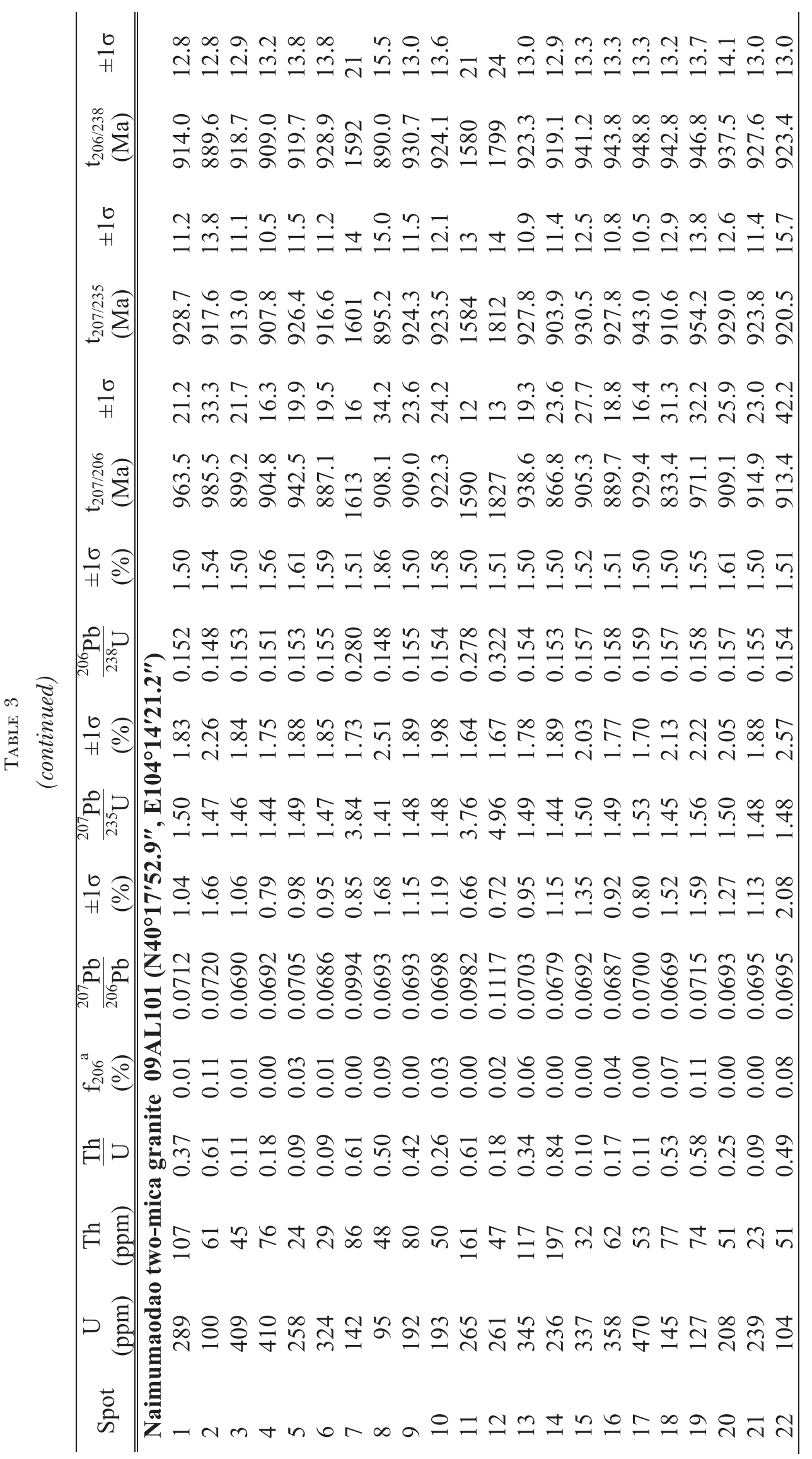




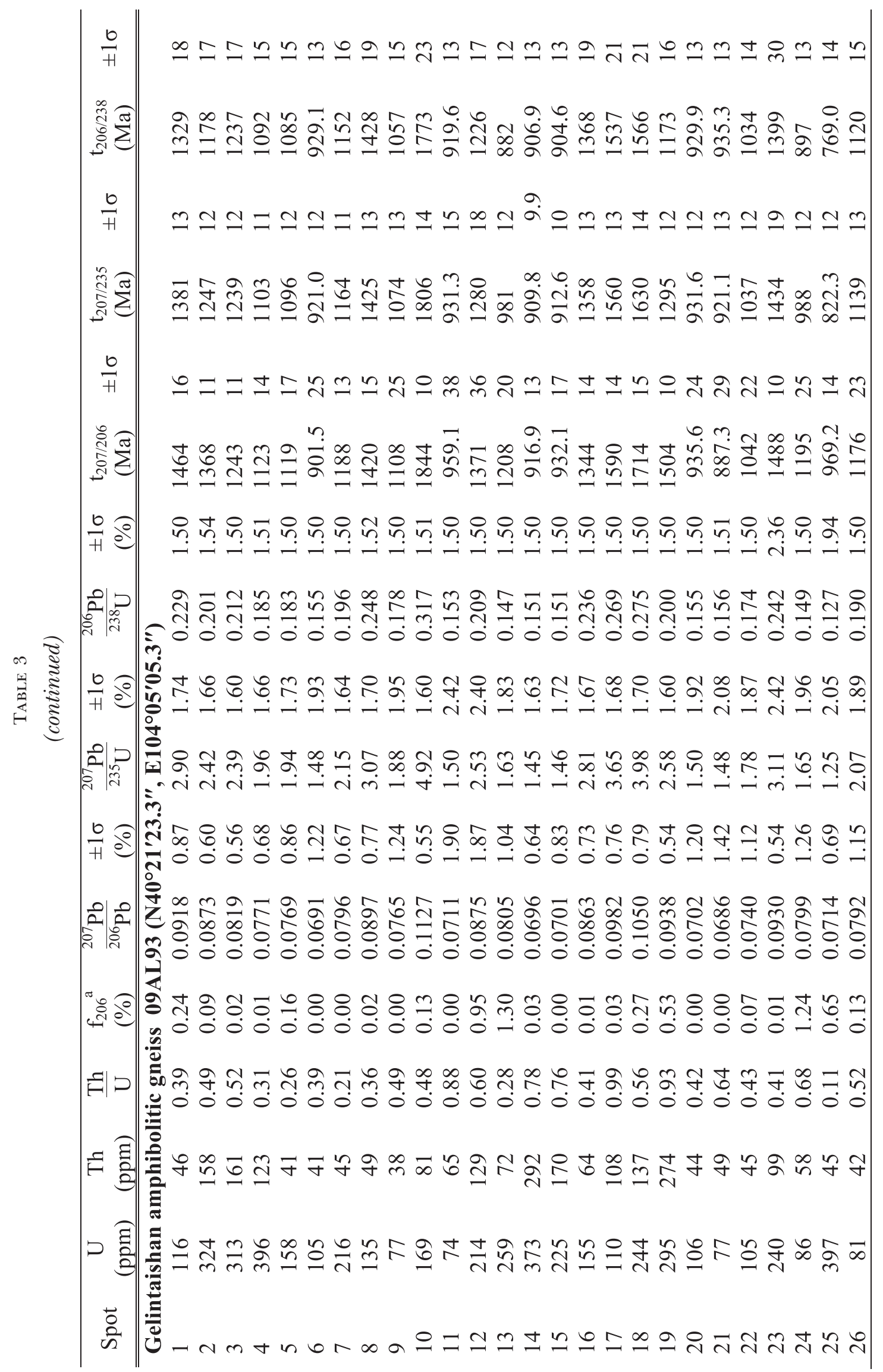




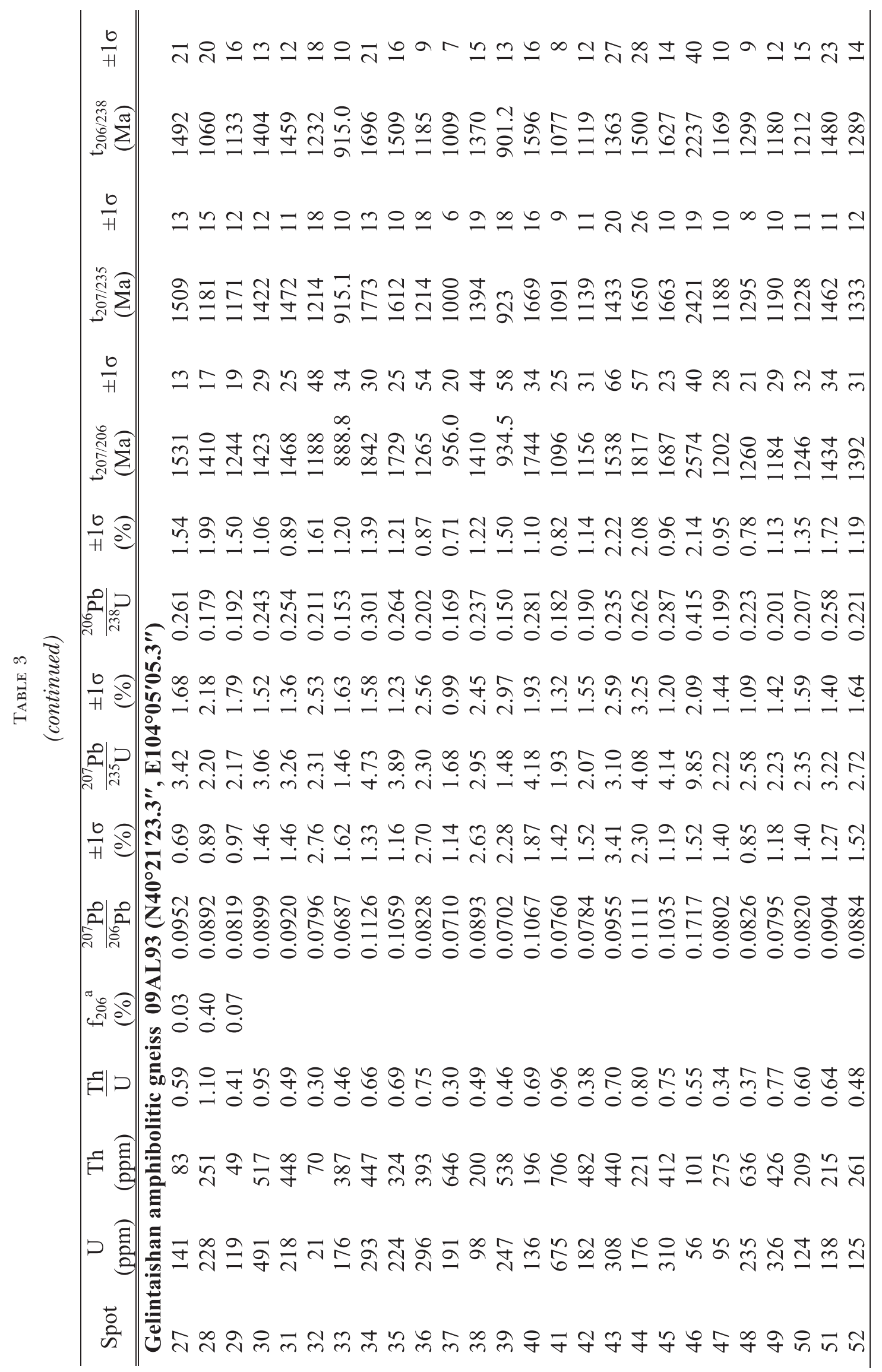




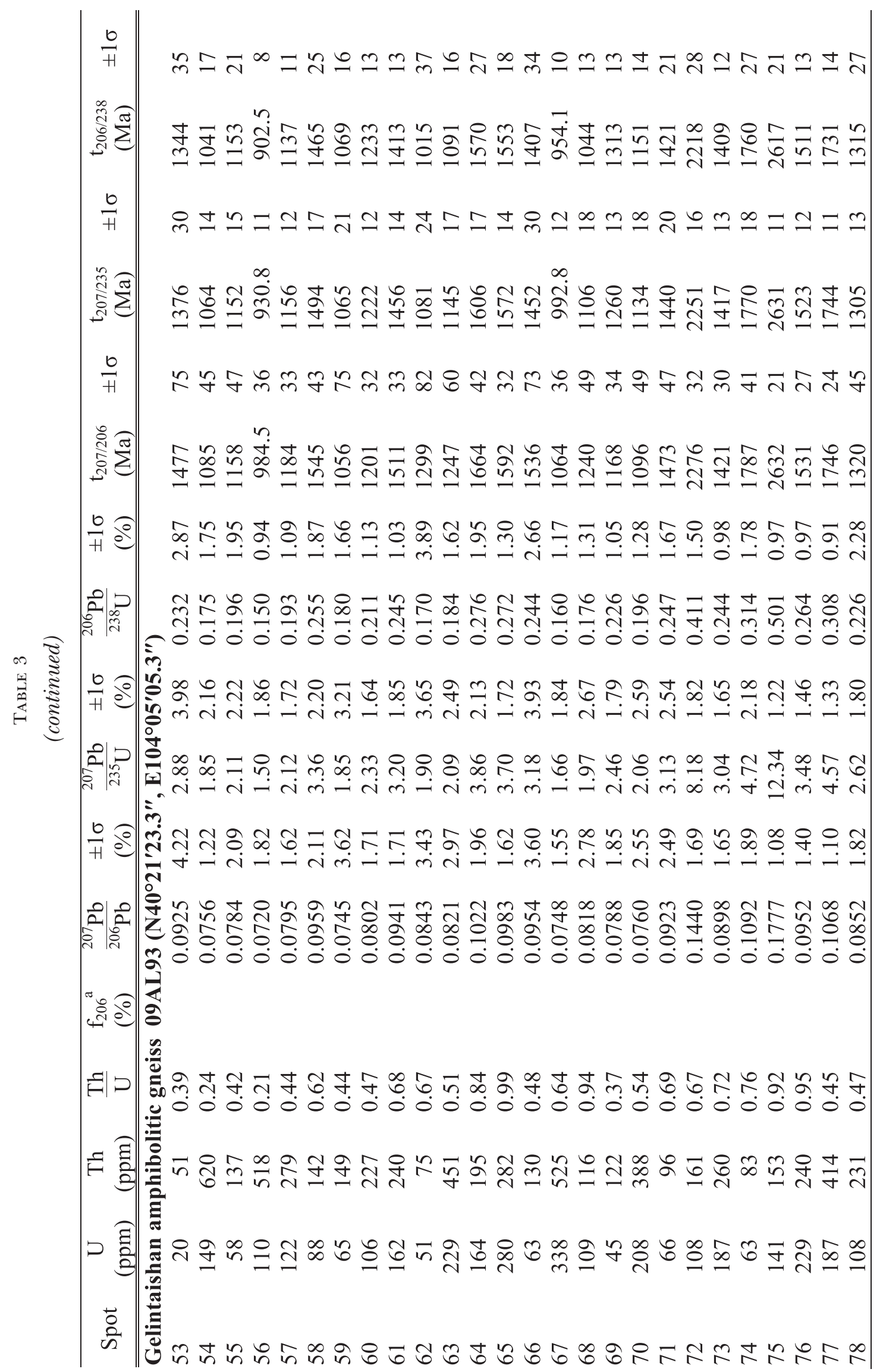




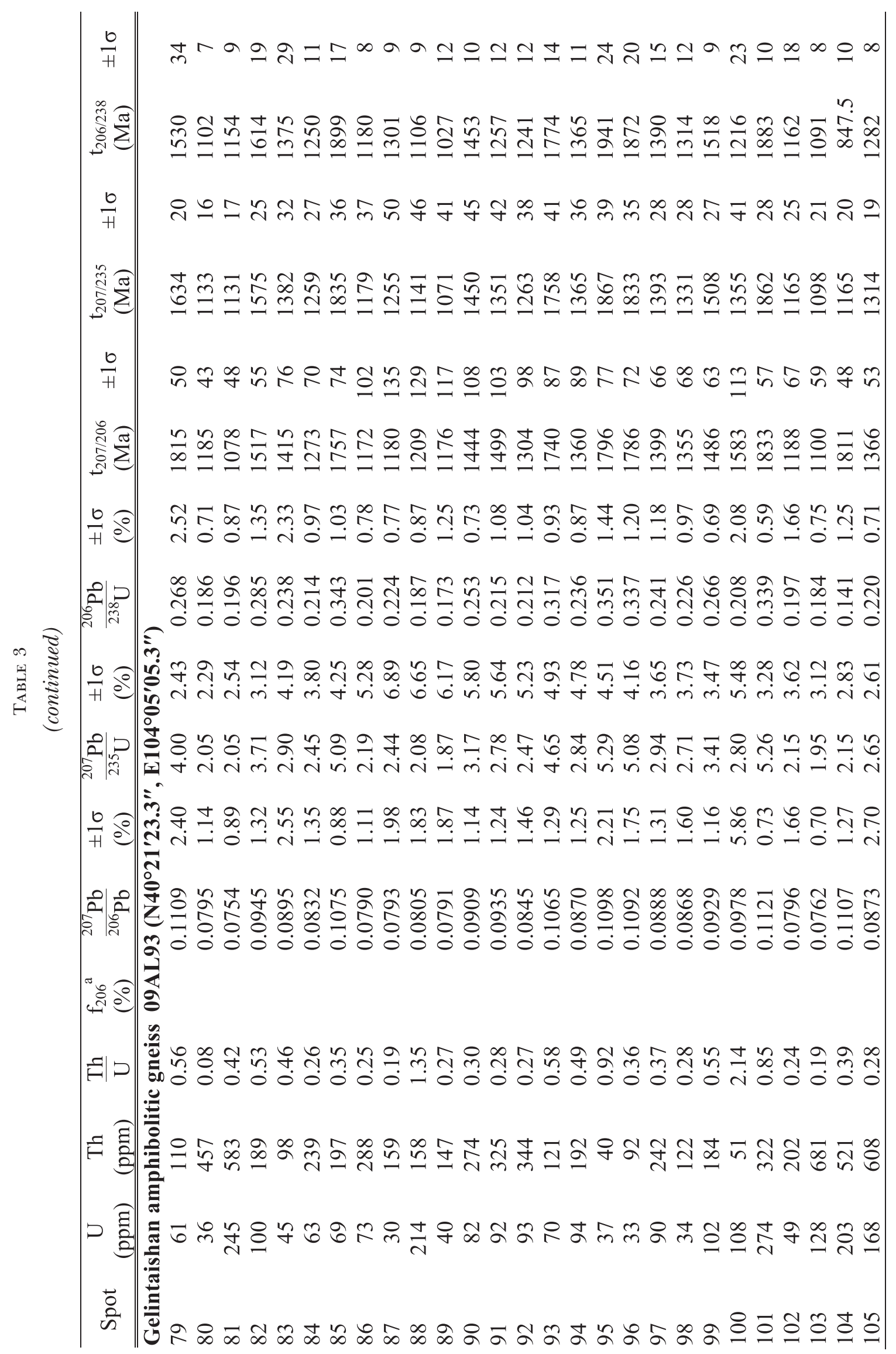




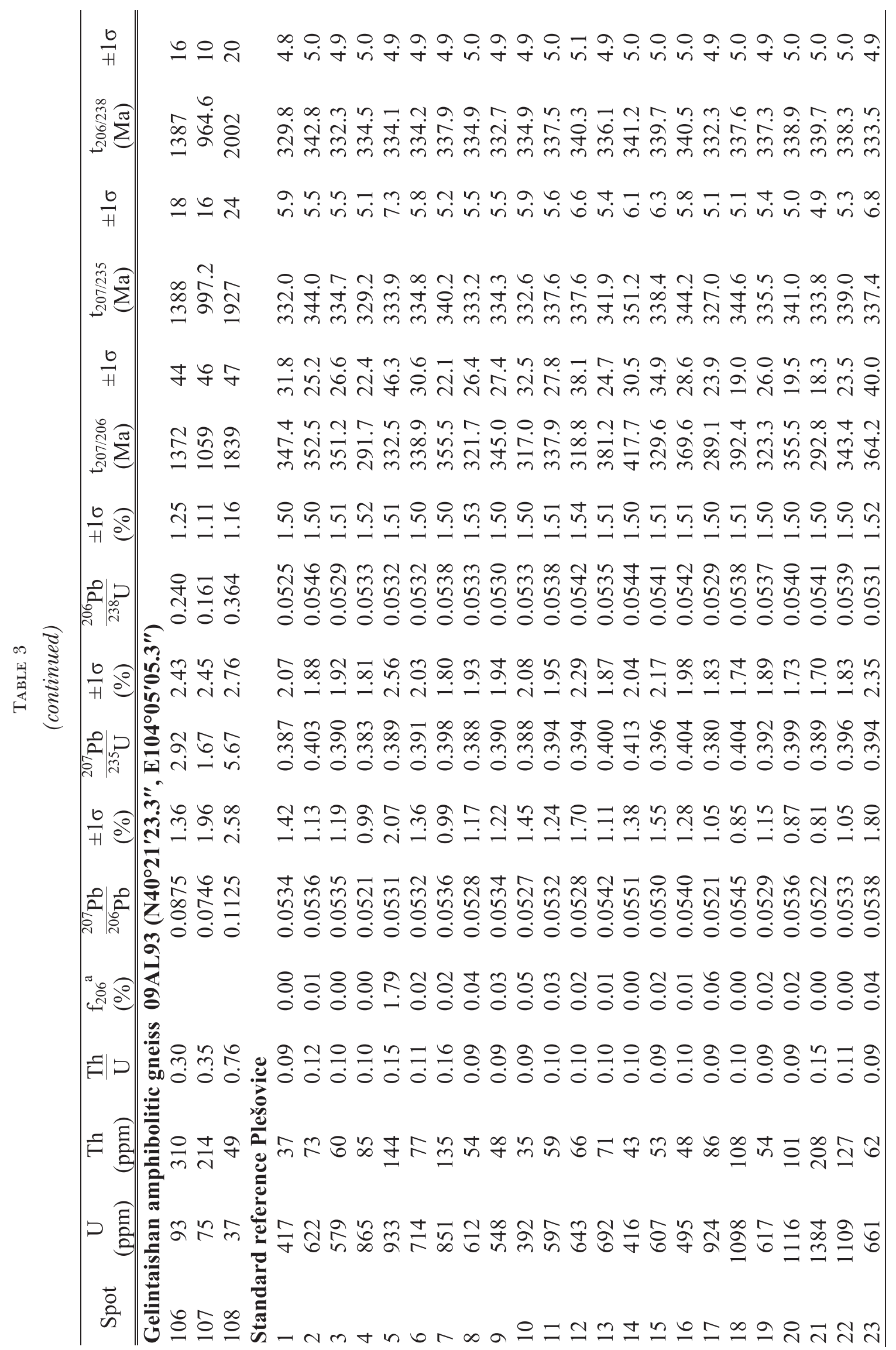


Westernmost North China and tectonic implications

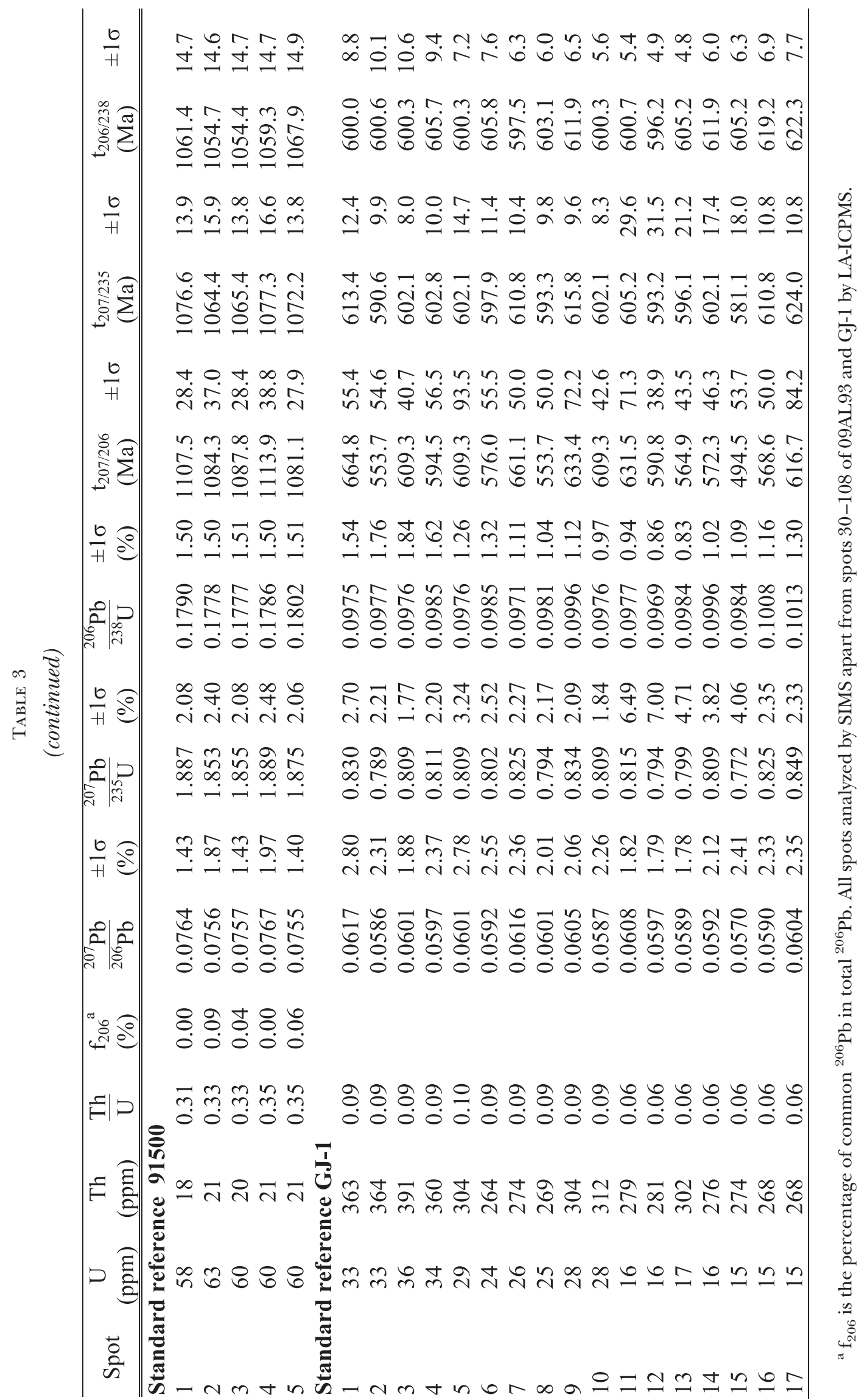


proposed by Anderson (2002). The weighted mean U-Pb ages and Concordia plots were processed using Isoplot/Ex v.3.0 program (Ludwig, 2003). Analyses of the zircon standard GJ-1 as an unknown yielded a weighted mean ${ }^{206} \mathrm{~Pb} /{ }^{238} \mathrm{U}$ age of $605 \pm 4 \mathrm{Ma}$ $(2 \sigma, \mathrm{n}=17)$, which is in good agreement with the recommended value (Jackson and others, 2004). LA-ICPMS zircon U-Pb isotopic data are presented in table 3.

\section{Zircon Oxygen Isotopes}

Zircon oxygen isotopes were measured using the same Cameca IMS-1280 SIMS at IGG-CAS. The original mounts were re-ground and polished to remove any trace of the analytical pits after U-Pb dating. Measurements were made using a primary beam of ${ }^{133} \mathrm{Cs}^{+}$ions accelerated at $10 \mathrm{kV}$, with an intensity of $\sim 2 \mathrm{nA}$, focused to a diameter of approximately $10 \mu \mathrm{m}$ on the same area of the grains that were previously analyzed for dating. An electron flood gun was used for charge compensation during analysis. Oxygen isotopes, ${ }^{18} \mathrm{O}$ and ${ }^{16} \mathrm{O}$, were measured simultaneously in multi-collector mode using two off-axis Faraday cups. The detailed analytical procedures were similar to those described by Li and others (2010b).

The measured oxygen isotopic data were corrected for instrumental mass fractionation (IMF) using the Penglai zircon standard $\left(\delta^{18} \mathrm{O}_{\text {vSMOw }}=5.3 \% 0\right)$ ( $\mathrm{Li}$ and others, 2010c). The internal precision of a single analysis was generally better than 0.2 permil ( $1 \sigma$ standard error) for the ${ }^{18} \mathrm{O} /{ }^{16} \mathrm{O}$ ratio. The external precision, measured by the reproducibility of repeated analyses of Penglai standard is better than 0.50 permil $(2 \mathrm{SD}, \mathrm{n}=58)$.

Five measurements of the zircon standard 91500 during the course of this study yielded a weighted mean of $\delta^{18} \mathrm{O}=10.3 \pm 0.4$ permil (2SD), which is consistent within errors with the reported value of $9.9 \pm 0.3$ permil (Wiedenbeck and others, 2004). Zircon oxygen isotopic data are listed in table 4.

\section{Zircon Lu-Hf Isotopes}

In situ zircon Lu-Hf isotopic analyses were carried out on a Neptune multicollector ICP-MS equipped with a Geolas-193 laser-ablation system at IGG-CAS. Lu-Hf isotopic analyses were conducted on the same zircon grains that were previously analyzed for U-Pb and $\mathrm{O}$ isotopes, with ablation pits of $60 \mu \mathrm{m}$ or $44 \mu \mathrm{m}$ in diameter, ablation time of 26 seconds, repetition rate of $8 \mathrm{~Hz}$, and laser beam energy density of $10 \mathrm{~J} / \mathrm{cm}^{2}$. Detailed analytical procedures were similar to those described by $\mathrm{Wu}$ and others (2006). Measured ${ }^{176} \mathrm{Hf} /{ }^{177} \mathrm{Hf}$ ratios were normalized to ${ }^{179} \mathrm{Hf} /{ }^{177} \mathrm{Hf}=0.7325$. Further external adjustment was not applied for the unknowns because our determined ${ }^{176} \mathrm{Hf} /{ }^{177} \mathrm{Hf}$ ratios for zircon standards Mud Tank $(0.282496 \pm 0.000005)$ and GJ-1 (0.282003 \pm 0.000008$)$ were in good agreement within errors with the reported values (Woodhead and Hergt, 2005; Griffin and others, 2006; Morel and others, 2008). Zircon Hf isotopic data are listed in table 4.

RESULTS

\section{Whole-rock Geochemical Characteristics}

The Dabusushan and Naimumaodao granites.-The gneissic two-mica granites from the Dabusushan and Naimumaodao plutons show similar geochemical characteristics. They are notably high in $\mathrm{SiO}_{2}$ ranging from 73.9 to 80.0 weight percent, but low in $\mathrm{TiO}_{2}, \mathrm{Fe}_{2} \mathrm{O}_{3}{ }^{\mathrm{T}}, \mathrm{MgO}$ and $\mathrm{CaO}$ (table 1 ). These samples have highly variable abundances of $\mathrm{K}_{2} \mathrm{O}\left(0.89-7.95\right.$ wt. \%) and $\mathrm{Na}_{2} \mathrm{O}\left(2.06-4.83\right.$ wt. \%), and the total $\mathrm{K}_{2} \mathrm{O}+\mathrm{Na}_{2} \mathrm{O}$ ranging from 4.71 to 10.01 weight percent, and most of them have $\mathrm{K}_{2} \mathrm{O}>\mathrm{Na}_{2} \mathrm{O}$. Their $\mathrm{A} / \mathrm{CNK}$ $\left[\right.$ molar $\left.\mathrm{Al}_{2} \mathrm{O}_{3} /\left(\mathrm{CaO}+\mathrm{Na}_{2} \mathrm{O}+\mathrm{K}_{2} \mathrm{O}\right)\right]$ values range from 0.95 to 1.56 , and most of them plot into the peraluminous field (fig. $5 \mathrm{~B}$ ).

The granites have similar chondrite-normalized REE patterns showing light rare earth element (LREE) enrichment and strong negative Eu anomalies, apart from 
TABLE 4

In situ zircon Hf-O isotopic results for the Neoproterozoic rocks from Alxa Block

\begin{tabular}{|c|c|c|c|c|c|c|c|c|c|}
\hline $\begin{array}{c}\text { Sample } \\
\text { Spot }\end{array}$ & $\begin{array}{l}\text { Age } \\
(\mathrm{Ma})\end{array}$ & $\frac{{ }^{176} \mathrm{Lu}}{{ }^{177} \mathrm{Hf}}$ & $\frac{{ }^{176} \mathrm{Hf}}{{ }^{177} \mathrm{Hf}}$ & $2 \sigma$ & $\varepsilon_{\mathrm{Hf}}(\mathrm{t})$ & $2 \sigma$ & $\begin{array}{l}\mathrm{T}_{\mathrm{DM}}{ }^{\mathrm{C}} \\
(\mathrm{Ma})\end{array}$ & $\begin{array}{l}\delta^{18} \mathrm{O} \\
(\%)\end{array}$ & $\pm 2 \sigma$ \\
\hline \multicolumn{10}{|c|}{ 09AL53 Dabusushan two-mica granite } \\
\hline 1.1 & 914 & 0.000953 & 0.282222 & 0.000016 & 0.1 & 0.6 & 1785 & 8.23 & 0.24 \\
\hline 1.2 & 914 & 0.001548 & 0.282274 & 0.000017 & 1.6 & 0.6 & 1691 & n.d. & \\
\hline 2.1 & 914 & 0.000829 & 0.282247 & 0.000015 & 1.1 & 0.5 & 1724 & 11.37 & 0.21 \\
\hline 3.1 & 914 & 0.000530 & 0.282156 & 0.000016 & -1.9 & 0.6 & 1916 & 11.17 & 0.32 \\
\hline 4.1 & 914 & 0.001895 & 0.282259 & 0.000017 & 0.9 & 0.6 & 1737 & 9.65 & 0.31 \\
\hline 5.1 & 914 & 0.001189 & 0.282246 & 0.000014 & 0.8 & 0.5 & 1741 & 10.73 & 0.19 \\
\hline 6.1 & 960 & 0.001877 & 0.282231 & 0.000019 & 0.9 & 0.7 & 1774 & 10.44 & 0.17 \\
\hline 7.1 & 914 & 0.001551 & 0.282245 & 0.000017 & 0.6 & 0.6 & 1756 & 10.67 & 0.29 \\
\hline 8.1 & 914 & 0.000727 & 0.282242 & 0.000018 & 1.0 & 0.6 & 1730 & 11.44 & 0.12 \\
\hline 9.1 & 914 & 0.000750 & 0.282172 & 0.000016 & -1.5 & 0.6 & 1890 & 12.10 & 0.25 \\
\hline 10.1 & 914 & 0.001372 & 0.282280 & 0.0000 & 1.9 & 0.5 & 1671 & 9.68 & 0.18 \\
\hline 11.1 & 914 & 0.000294 & 0.282111 & 0.000017 & -3.4 & 0.6 & 2008 & 10.64 & 0.20 \\
\hline 12.1 & 914 & 0.000923 & 0.282187 & 0.000015 & -1.1 & 0.5 & 1863 & 11.15 & 0.23 \\
\hline 13.1 & 914 & 0.000841 & 0.282192 & 0.000017 & -0.8 & 0.6 & 1848 & 11.60 & 0.24 \\
\hline 14.1 & 914 & 0.000265 & 0.282155 & 0.000014 & -1.8 & 0.5 & 1909 & 11.14 & 0.24 \\
\hline 15.1 & 914 & 0.001790 & 0.282236 & 0.000 & 0.1 & 0.6 & 1785 & 9.49 & 0.24 \\
\hline 16.1 & 914 & 0.002019 & 0.282233 & 0.000018 & -0.1 & 0.6 & 1802 & 9.48 & 0.27 \\
\hline 17.1 & 914 & 0.001098 & 0.282327 & 0.000 & 3.8 & 0.7 & 1552 & 10.46 & 0.24 \\
\hline 18.1 & 914 & 0.000470 & 0.282209 & 0.000012 & 0.0 & 0.4 & 1796 & 10.91 & 0.20 \\
\hline \multicolumn{10}{|c|}{ 09AL62 Dabusushan two-mica granite } \\
\hline 1 & 909 & 0.000416 & 0.282092 & & -4.2 & 0.4 & 2058 & 9.38 & 0.24 \\
\hline 2 & 909 & 0.000562 & 0.282113 & 0.000014 & -3.6 & 0.5 & 2018 & 9.45 & 0.26 \\
\hline 3 & 909 & 0.000972 & 0.282228 & 0.000016 & 0.3 & 0.6 & 1774 & 10.96 & 0.28 \\
\hline 4 & 909 & 0.001467 & 0.282141 & 0.000017 & -3.1 & 0.6 & 1988 & 9.61 & 0.19 \\
\hline 5 & 909 & 0.001475 & 0.282190 & 0.000014 & -1.4 & 0.5 & 1880 & 10.62 & 0.20 \\
\hline 6 & 909 & 0.001270 & 0.282156 & 0.000 & -2.5 & 0.6 & 1948 & 10.19 & 0.20 \\
\hline 7 & 909 & 0.001425 & 0.282159 & 0.000016 & -2.5 & 0.6 & 1948 & 9.23 & 0.23 \\
\hline 8 & 909 & 0.001281 & 0.282148 & 0.000018 & -2.8 & 0.6 & 1966 & 9.69 & 0.24 \\
\hline 9 & 909 & 0.001144 & 0.282162 & 0.000015 & -2.2 & 0.5 & 1931 & 9.96 & 0.30 \\
\hline 10 & 909 & 0.000734 & 0.282137 & 0.000017 & -2.8 & 0.6 & 1970 & 10.75 & 0.14 \\
\hline 11 & 90 & 0.000376 & 0.282124 & 0.00 & -3.1 & 0.8 & 1986 & 10.86 & 0.21 \\
\hline 12 & 909 & 0.000995 & 0.282167 & 0.000015 & -1.9 & 0.5 & 1913 & 10.58 & 0.37 \\
\hline 13 & 909 & 0.001165 & 0.282193 & 0.000014 & -1.1 & 0.5 & 1860 & 9.93 & 0.19 \\
\hline 14 & 909 & 0.002368 & 0.282267 & 0.000020 & 0.8 & 0.7 & 1741 & 12.08 & 0.19 \\
\hline 15 & 909 & 0.001607 & 0.282139 & 0.000017 & -3.3 & 0.6 & 1999 & 11.22 & 0.22 \\
\hline 16 & 909 & 0.000686 & 0.282041 & & -6.2 & 0.5 & 2184 & 10.46 & 0.15 \\
\hline 17 & $90 s$ & 0.000988 & 0.282125 & 0.000015 & -3 & 0.5 & 2008 & 10.67 & 0.24 \\
\hline 18 & 909 & 0.001451 & 0.282193 & 0.000017 & -1.3 & 0.6 & 1871 & 10.35 & 0.27 \\
\hline 19 & 909 & 0.000593 & 0.282140 & 0.000014 & -2.6 & 0.5 & 1959 & 10.41 & 0.11 \\
\hline 20 & 909 & 0.000728 & 0.282127 & 0.000013 & -3.2 & 0.5 & 1992 & 10.58 & 0.24 \\
\hline \multicolumn{10}{|c|}{ 09AL101 Naimumaodao two-mica granite } \\
\hline 1 & 929 & 0.001341 & 0.282143 & 0.0000 & -2.5 & 0.5 & 1968 & 10.58 & 0.19 \\
\hline 2 & 929 & 0.001295 & 0.282209 & 0.000014 & -0.2 & 0.5 & 1818 & 9.49 & 0.18 \\
\hline 3 & 929 & 0.000939 & 0.282206 & 0.000013 & -0.1 & 0.4 & 1811 & 11.26 & 0.22 \\
\hline 4 & 929 & 0.001364 & 0.282159 & 0.000011 & -2.0 & 0.4 & 1934 & 11.89 & 0.21 \\
\hline 5 & 929 & 0.000698 & 0.282169 & 0.000012 & -1.2 & 0.4 & 1886 & 11.77 & 0.13 \\
\hline 6 & 929 & 0.001288 & 0.282181 & 0.000014 & -1.2 & 0.5 & 1881 & 11.41 & 0.24 \\
\hline
\end{tabular}


TABLE 4

(continued)

\begin{tabular}{|c|c|c|c|c|c|c|c|c|c|}
\hline $\begin{array}{c}\text { Sample } \\
\text { Spot }\end{array}$ & $\begin{array}{l}\text { Age } \\
(\mathrm{Ma})\end{array}$ & $\frac{{ }^{176} \mathrm{Lu}}{{ }^{177} \mathrm{Hf}}$ & $\frac{{ }^{176} \mathrm{Hf}}{{ }^{177} \mathrm{Hf}}$ & $2 \sigma$ & $\varepsilon_{\mathrm{Hf}}(\mathrm{t})$ & $2 \sigma$ & $\begin{array}{l}\mathrm{T}_{\mathrm{DM}}{ }^{\mathrm{C}} \\
(\mathrm{Ma})\end{array}$ & $\begin{array}{l}\delta^{18} \mathrm{O} \\
(\% \mathrm{o})\end{array}$ & $\pm 2 \sigma$ \\
\hline \multicolumn{10}{|c|}{ 09AL101 Naimumaodao two-mica granite } \\
\hline 7 & 1613 & 0.001833 & 0.281965 & 0.000013 & 5.4 & 0.5 & 1993 & 9.73 & 0.12 \\
\hline 8 & 929 & 0.001710 & 0.282187 & 0.000021 & -1.2 & 0.7 & 1884 & 8.60 & 0.20 \\
\hline 9 & 929 & 0.000649 & 0.282188 & 0.000038 & -0.5 & 1.3 & 1841 & 12.07 & 0.20 \\
\hline 10 & 929 & 0.000412 & 0.282206 & 0.000014 & 0.3 & 0.5 & 1790 & 9.77 & 0.21 \\
\hline 11 & 1590 & 0.000936 & 0.281932 & 0.000019 & 4.6 & 0.7 & 2022 & 6.26 & 0.21 \\
\hline 12 & 1827 & 0.001181 & 0.281736 & 0.000015 & 2.6 & 0.5 & 2334 & 8.22 & 0.22 \\
\hline 13 & 929 & 0.002636 & 0.282239 & 0.000018 & 0.1 & 0.6 & 1803 & 11.41 & 0.24 \\
\hline 14 & 929 & 0.001614 & 0.282219 & 0.000016 & 0.0 & 0.6 & 1808 & 8.60 & 0.19 \\
\hline 15 & 929 & 0.000746 & 0.282212 & 0.000016 & 0.3 & 0.6 & 1790 & 11.29 & 0.16 \\
\hline 16 & 929 & 0.000980 & 0.282127 & 0.000015 & -2.9 & 0.5 & 1989 & 11.63 & 0.22 \\
\hline 17 & 929 & 0.001646 & 0.282205 & 0.000022 & -0.6 & 0.8 & 1841 & 12.13 & 0.16 \\
\hline 18 & 929 & 0.000615 & 0.282184 & 0.000013 & -0.6 & 0.5 & 1847 & 11.13 & 0.19 \\
\hline 19 & 929 & 0.001322 & 0.282174 & 0.000013 & -1.4 & 0.4 & 1898 & 10.15 & 0.20 \\
\hline 20 & 929 & 0.000889 & 0.282164 & 0.000014 & -1.5 & 0.5 & 1903 & 11.08 & 0.18 \\
\hline 21 & 929 & 0.000300 & 0.282182 & 0.000011 & -0.5 & 0.4 & 1839 & n.d. & \\
\hline 22 & 929 & 0.002665 & 0.282192 & 0.000011 & -1.7 & 0.4 & 1911 & n.d. & \\
\hline \multicolumn{10}{|c|}{ 09AL93 Gelintaishan amphibolitic gneiss } \\
\hline 1 & 1464 & 0.000930 & 0.282046 & 0.000025 & 6.0 & 0.9 & 1842 & 5.20 & 0.20 \\
\hline 2 & 1368 & 0.002090 & 0.282218 & 0.000029 & 8.9 & 1.0 & 1581 & 11.66 & 0.21 \\
\hline 3 & 1243 & 0.000961 & 0.282115 & 0.000024 & 3.5 & 0.8 & 1827 & 6.19 & 0.29 \\
\hline 4 & 1123 & 0.001714 & 0.282271 & 0.000026 & 5.9 & 0.9 & 1583 & 10.84 & 0.22 \\
\hline 5 & 1119 & 0.000684 & 0.282257 & 0.000026 & 6.1 & 0.9 & 1568 & 10.05 & 0.23 \\
\hline 6 & 929 & 0.000562 & 0.282190 & 0.000026 & -0.4 & 0.9 & 1832 & 7.85 & 0.20 \\
\hline 7 & 1188 & 0.000984 & 0.282277 & 0.000027 & 8.0 & 1.0 & 1495 & 8.03 & 0.16 \\
\hline 8 & 1420 & 0.001469 & 0.282084 & 0.000027 & 5.8 & 1.0 & 1817 & 9.15 & 0.22 \\
\hline 9 & 1108 & 0.000799 & 0.282331 & 0.000028 & 8.4 & 1.0 & 1411 & 7.71 & 0.18 \\
\hline 10 & 1844 & 0.000200 & 0.281462 & 0.000027 & -5.5 & 1.0 & 2856 & 8.17 & 0.19 \\
\hline 11 & 920 & 0.001345 & 0.282188 & 0.000027 & -1.1 & 1.0 & 1926 & 10.09 & 0.16 \\
\hline 12 & 1371 & 0.001548 & 0.282209 & 0.000032 & 9.1 & 1.1 & 1566 & 6.65 & 0.18 \\
\hline 13 & 1208 & 0.002500 & 0.282146 & 0.000023 & 2.6 & 0.8 & 1858 & 10.33 & 0.19 \\
\hline 14 & 907 & 0.001865 & 0.282124 & 0.000029 & -4.0 & 1.0 & 2044 & 7.67 & 0.20 \\
\hline 15 & 905 & 0.000789 & 0.282223 & 0.000029 & 0.1 & 1.0 & 1782 & 7.40 & 0.26 \\
\hline 16 & 1344 & 0.001360 & 0.282256 & 0.000031 & 10.4 & 1.1 & 1465 & 6.74 & 0.16 \\
\hline 17 & 1590 & 0.001189 & 0.281966 & 0.000027 & 5.6 & 1.0 & 1961 & 5.52 & 0.19 \\
\hline 18 & 1714 & 0.001498 & 0.281761 & 0.000029 & 0.7 & 1.0 & 2370 & 5.57 & 0.18 \\
\hline 19 & 1504 & 0.000926 & 0.282071 & 0.000029 & 7.7 & 1.0 & 1760 & 5.92 & 0.18 \\
\hline 20 & 930 & 0.001034 & 0.282152 & 0.000020 & -2.0 & 0.7 & 1935 & 7.91 & 0.16 \\
\hline 21 & 935 & 0.000857 & 0.282278 & 0.000030 & 2.7 & 1.0 & 1641 & 7.33 & 0.19 \\
\hline 22 & 1042 & 0.000386 & 0.282182 & 0.000029 & 1.9 & 1.0 & 1772 & 8.27 & 0.19 \\
\hline 23 & 1488 & 0.001256 & 0.282072 & 0.000027 & 7.1 & 1.0 & 1788 & 8.36 & 0.25 \\
\hline 24 & 1195 & 0.000902 & 0.282036 & 0.000038 & -0.3 & 1.3 & 2032 & 5.54 & 0.17 \\
\hline 25 & 969 & 0.000280 & 0.282113 & 0.000029 & -2.0 & 1.0 & 1968 & 9.78 & 0.20 \\
\hline 26 & 1176 & 0.001208 & 0.282210 & 0.000025 & 5.3 & 0.9 & 1664 & 5.82 & 0.15 \\
\hline 27 & 1531 & 0.000618 & 0.281994 & 0.000021 & 5.9 & 0.7 & 1896 & 5.93 & 0.21 \\
\hline 28 & 1410 & 0.001461 & 0.282059 & 0.000031 & 4.7 & 1.1 & 1879 & 7.70 & 0.26 \\
\hline 29 & 1244 & 0.000326 & 0.282244 & 0.000028 & 8.7 & 1.0 & 1498 & 6.22 & 0.21 \\
\hline
\end{tabular}


TABle 4

(continued)

\begin{tabular}{|c|c|c|c|c|c|c|c|c|c|}
\hline $\begin{array}{c}\text { Sample } \\
\text { Spot }\end{array}$ & $\begin{array}{l}\text { Age } \\
(\mathrm{Ma})\end{array}$ & $\frac{{ }^{176} \mathrm{Lu}}{{ }^{177} \mathrm{Hf}}$ & $\frac{{ }^{176} \mathrm{Hf}}{{ }^{177} \mathrm{Hf}}$ & $2 \sigma$ & $\varepsilon_{\mathrm{Hf}}(\mathrm{t})$ & $2 \sigma$ & $\begin{array}{l}\mathrm{T}_{\mathrm{DM}}{ }^{\mathrm{C}} \\
(\mathrm{Ma})\end{array}$ & $\begin{array}{l}\delta^{18} \mathrm{O} \\
(\% \mathrm{o}) \\
\end{array}$ & $\pm 2 \sigma$ \\
\hline \multicolumn{10}{|c|}{ Standard reference GJ-1 } \\
\hline GJ-1 01 & & 0.000252 & 0.281960 & 0.000018 & & & & & \\
\hline GJ-1 02 & & 0.000245 & 0.282010 & 0.000014 & & & & & \\
\hline GJ-1 03 & & 0.000254 & 0.281986 & 0.000017 & & & & & \\
\hline GJ-1 04 & & 0.000251 & 0.282015 & 0.000014 & & & & & \\
\hline GJ-1 05 & & 0.000256 & 0.281999 & 0.000016 & & & & & \\
\hline GJ-1 06 & & 0.000247 & 0.282037 & 0.000015 & & & & & \\
\hline GJ-1 07 & & 0.000254 & 0.282024 & 0.000015 & & & & & \\
\hline GJ-1 08 & & 0.000251 & 0.281999 & 0.000017 & & & & & \\
\hline GJ-1 09 & & 0.000262 & 0.282025 & 0.000016 & & & & & \\
\hline GJ-1 10 & & 0.000248 & 0.282027 & 0.000015 & & & & & \\
\hline GJ-1 11 & & 0.000234 & 0.282021 & 0.000015 & & & & & \\
\hline GJ-1 12 & & 0.000240 & 0.281992 & 0.000013 & & & & & \\
\hline GJ-1 13 & & 0.000251 & 0.281995 & 0.000013 & & & & & \\
\hline GJ-1 14 & & 0.000236 & 0.282011 & 0.000012 & & & & & \\
\hline GJ-1 15 & & 0.000251 & 0.281974 & 0.000019 & & & & & \\
\hline GJ-1 16 & & 0.000254 & 0.282000 & 0.000019 & & & & & \\
\hline GJ-1 17 & & 0.000257 & 0.281994 & 0.000017 & & & & & \\
\hline GJ-1 18 & & 0.000235 & 0.282012 & 0.000017 & & & & & \\
\hline GJ-1 19 & & 0.000253 & 0.281988 & 0.000017 & & & & & \\
\hline GJ-1 20 & & 0.000254 & 0.281994 & 0.000011 & & & & & \\
\hline GJ-1 21 & & 0.000259 & 0.281984 & 0.000019 & & & & & \\
\hline GJ-1 22 & & 0.000256 & 0.281983 & 0.000018 & & & & & \\
\hline GJ-1 23 & & 0.000253 & 0.282012 & 0.000020 & & & & & \\
\hline \multicolumn{10}{|c|}{ Standard reference Mud Tank } \\
\hline MUD 01 & & 0.000046 & 0.282489 & 0.000013 & & & & & \\
\hline MUD 02 & & 0.000042 & 0.282499 & 0.000012 & & & & & \\
\hline MUD 03 & & 0.000044 & 0.282480 & 0.000013 & & & & & \\
\hline MUD 04 & & 0.000045 & 0.282480 & 0.000013 & & & & & \\
\hline MUD 05 & & 0.000086 & 0.282502 & 0.000013 & & & & & \\
\hline MUD 06 & & 0.000046 & 0.282495 & 0.000012 & & & & & \\
\hline MUD 07 & & 0.000033 & 0.282501 & 0.000012 & & & & & \\
\hline MUD 08 & & 0.000051 & 0.282508 & 0.000013 & & & & & \\
\hline MUD 09 & & 0.000033 & 0.282507 & 0.000012 & & & & & \\
\hline MUD 10 & & 0.000032 & 0.282476 & 0.000010 & & & & & \\
\hline MUD 11 & & 0.000047 & 0.282491 & 0.000011 & & & & & \\
\hline MUD 12 & & 0.000048 & 0.282495 & 0.000009 & & & & & \\
\hline MUD 13 & & 0.000050 & 0.282491 & 0.000011 & & & & & \\
\hline MUD 14 & & 0.000034 & 0.282498 & 0.000010 & & & & & \\
\hline MUD 15 & & 0.000032 & 0.282485 & 0.000014 & & & & & \\
\hline MUD 16 & & 0.000046 & 0.282493 & 0.000014 & & & & & \\
\hline MUD 17 & & 0.000038 & 0.282485 & 0.000016 & & & & & \\
\hline MUD 18 & & 0.000037 & 0.282514 & 0.000015 & & & & & \\
\hline MUD 19 & & 0.000046 & 0.282514 & 0.000013 & & & & & \\
\hline MUD 20 & & 0.000044 & 0.282510 & 0.000015 & & & & & \\
\hline MUD 21 & & 0.000032 & 0.282507 & 0.000013 & & & & & \\
\hline MUD 22 & & 0.000044 & 0.282504 & 0.000012 & & & & & \\
\hline
\end{tabular}


TABLE 4

(continued)

\begin{tabular}{|c|c|c|c|c|c|c|c|c|c|}
\hline $\begin{array}{l}\text { Sample } \\
\text { Spot }\end{array}$ & $\begin{array}{l}\text { Age } \\
(\mathrm{Ma})\end{array}$ & $\frac{{ }^{176} \mathrm{Lu}}{{ }^{177} \mathrm{Hf}}$ & $\frac{{ }^{176} \mathrm{Hf}}{{ }^{177} \mathrm{Hf}}$ & $2 \sigma$ & $\varepsilon_{\mathrm{Hf}}(\mathrm{t})$ & $2 \sigma$ & $\begin{array}{l}\mathrm{T}_{\mathrm{DM}}{ }^{\mathrm{C}} \\
(\mathrm{Ma})\end{array}$ & $\begin{array}{l}\delta^{18} \mathrm{O} \\
(\%)\end{array}$ & $\pm 2 \sigma$ \\
\hline \multicolumn{10}{|c|}{ Standard reference Penglai } \\
\hline Penglai 01 & & & & & & & & 5.26 & 0.26 \\
\hline Penglai 02 & & & & & & & & 5.39 & 0.17 \\
\hline Penglai 03 & & & & & & & & 5.26 & 0.32 \\
\hline Penglai 04 & & & & & & & & 5.29 & 0.20 \\
\hline Penglai 05 & & & & & & & & 5.27 & 0.20 \\
\hline Penglai 06 & & & & & & & & 5.31 & 0.19 \\
\hline Penglai 07 & & & & & & & & 5.31 & 0.23 \\
\hline Penglai 08 & & & & & & & & 5.26 & 0.21 \\
\hline Penglai 09 & & & & & & & & 5.31 & 0.20 \\
\hline Penglai 10 & & & & & & & & 5.33 & 0.23 \\
\hline Penglai 11 & & & & & & & & 5.42 & 0.22 \\
\hline Penglai 12 & & & & & & & & 5.13 & 0.16 \\
\hline Penglai 13 & & & & & & & & 5.30 & 0.23 \\
\hline Penglai 14 & & & & & & & & 5.33 & 0.20 \\
\hline Penglai 15 & & & & & & & & 5.31 & 0.23 \\
\hline Penglai 16 & & & & & & & & 5.38 & 0.18 \\
\hline Penglai 17 & & & & & & & & 5.22 & 0.18 \\
\hline Penglai 18 & & & & & & & & 5.14 & 0.27 \\
\hline Penglai 19 & & & & & & & & 5.66 & 0.20 \\
\hline Penglai 20 & & & & & & & & 5.10 & 0.19 \\
\hline Penglai 21 & & & & & & & & 5.34 & 0.26 \\
\hline Penglai 22 & & & & & & & & 5.40 & 0.21 \\
\hline Penglai 23 & & & & & & & & 5.18 & 0.23 \\
\hline Penglai 24 & & & & & & & & 5.09 & 0.20 \\
\hline Penglai 25 & & & & & & & & 5.25 & 0.15 \\
\hline Penglai 26 & & & & & & & & 5.44 & 0.23 \\
\hline Penglai 27 & & & & & & & & 5.41 & 0.30 \\
\hline Penglai 28 & & & & & & & & 5.38 & 0.22 \\
\hline Penglai 29 & & & & & & & & 5.40 & 0.20 \\
\hline Penglai 30 & & & & & & & & 5.34 & 0.17 \\
\hline Penglai 31 & & & & & & & & 5.07 & 0.20 \\
\hline Penglai 32 & & & & & & & & 5.09 & 0.26 \\
\hline Penglai 33 & & & & & & & & 5.36 & 0.20 \\
\hline Penglai 34 & & & & & & & & 5.40 & 0.24 \\
\hline Penglai 35 & & & & & & & & 5.31 & 0.26 \\
\hline Penglai 36 & & & & & & & & 5.35 & 0.27 \\
\hline Penglai 37 & & & & & & & & 5.39 & 0.23 \\
\hline Penglai 38 & & & & & & & & 5.20 & 0.22 \\
\hline Penglai 39 & & & & & & & & 5.53 & 0.22 \\
\hline Penglai 40 & & & & & & & & 5.06 & 0.25 \\
\hline Penglai 41 & & & & & & & & 5.22 & 0.19 \\
\hline Penglai 42 & & & & & & & & 5.15 & 0.26 \\
\hline Penglai 43 & & & & & & & & 5.45 & 0.24 \\
\hline Penglai 44 & & & & & & & & 5.36 & 0.18 \\
\hline Penglai 45 & & & & & & & & 5.39 & 0.22 \\
\hline Penglai 46 & & & & & & & & 5.51 & 0.27 \\
\hline Penglai 47 & & & & & & & & 5.24 & 0.15 \\
\hline Penglai 48 & & & & & & & & 5.25 & 0.24 \\
\hline
\end{tabular}


TABLE 4

(continued)

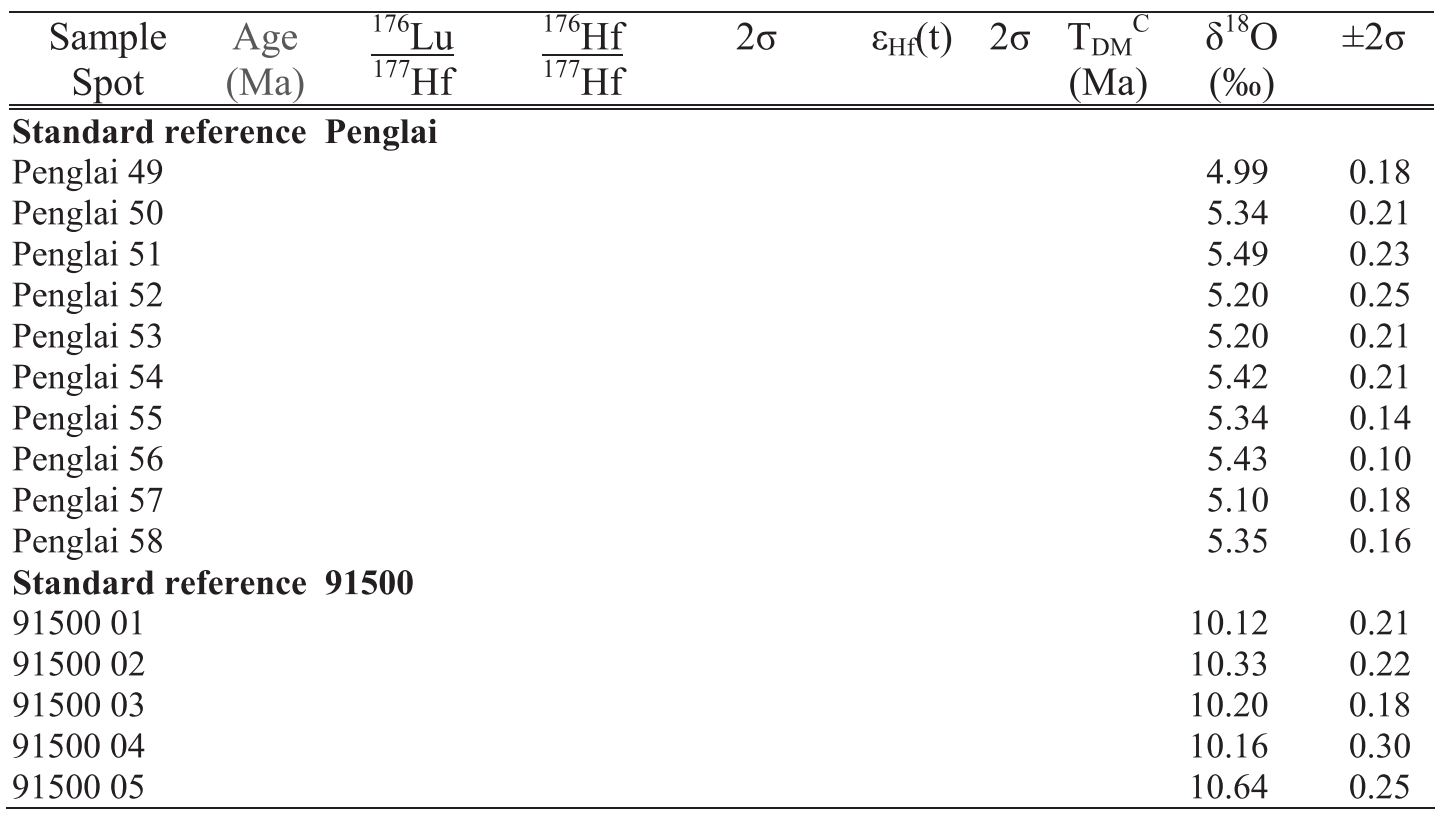

$$
\text { n.d. }=\text { not determined }
$$

$\varepsilon_{\mathrm{Hf}}(\mathrm{t})=10000\left\{\left[\left({ }^{176} \mathrm{Hf} /{ }^{177} \mathrm{Hf}\right)_{\mathrm{s}}-\left({ }^{176} \mathrm{Lu} /{ }^{177} \mathrm{Hf}\right)_{\mathrm{s}} \times\left(\mathrm{e}^{\lambda \mathrm{t}}-1\right)\right] /\left[\left({ }^{176} \mathrm{Hf} /{ }^{177} \mathrm{Hf}\right)_{\mathrm{CHUR}(0)}-\left({ }^{176} \mathrm{Lu} /\right.\right.\right.$ $\left.\left.{ }^{177} \mathrm{Hf}\right)_{\mathrm{CHUR}} \times\left(\mathrm{e}^{\lambda \mathrm{t}}-1\right)\right]-1 ; T_{\mathrm{DM}}=1 / \lambda \times \ln \left\{1+\left[\left({ }^{176} \mathrm{Hf} /{ }^{177} \mathrm{Hf}\right)_{\mathrm{s}}-\left({ }^{176} \mathrm{Hf} /{ }^{177} \mathrm{Hf}\right)_{\mathrm{DM}}\right] /\left[\left({ }^{176} \mathrm{Lu} /{ }^{177} \mathrm{Hf}\right)_{\mathrm{s}}-\right.\right.$ $\left.\left.\left({ }^{176} \mathrm{Lu} /{ }^{177} \mathrm{Hf}\right)_{\mathrm{DM}}\right]\right\} ; \mathrm{T}_{\mathrm{DM}}{ }^{\mathrm{C}}=\mathrm{T}_{\mathrm{DM}}-\left(\mathrm{T}_{\mathrm{DM}}-\mathrm{t}\right) \times\left[\mathrm{f}_{\mathrm{cc}}-\mathrm{f}_{\mathrm{s}} /\left(\mathrm{f}_{\mathrm{cc}}-\mathrm{f}_{\mathrm{DM}}\right)\right] ; f_{\mathrm{Lu} / \mathrm{Hf}}=\left({ }^{176} \mathrm{Lu} /{ }^{177} \mathrm{Hf}\right)_{\mathrm{s}} /\left({ }^{176} \mathrm{Lu} /\right.$ $\left.{ }^{177} \mathrm{Hf}\right)_{\text {CHUR }}-1$; where, $\mathrm{f}_{\mathrm{CG}}, \mathrm{f}_{\mathrm{S}}$ and $\mathrm{f}_{\mathrm{DM}}$ are the $\mathrm{f}_{\mathrm{Lu} / \mathrm{Hf}}$ values of the continental crust, zircon sample and the depleted mantle; subscript $\mathrm{S}=$ analyzed zircon sample, CHUR = chondritic uniform reservoir; DM = depleted mantle; $\mathrm{t}=$ crystallization time or metamorphic time of zircon; $\lambda=1.867 \times 10^{-1}$ year $^{-1}$, decay constant of ${ }^{176} \mathrm{Lu}$ (Soderlund and others, 2004); ${ }^{176} \mathrm{Hf} /{ }^{177} \mathrm{Hf}_{\mathrm{DM}}=0.28325 ;{ }^{176} \mathrm{Lu} /{ }^{177} \mathrm{Hf}_{\mathrm{DM}}=0.0384$; present-day ${ }^{176} \mathrm{Hf} /{ }^{177} \mathrm{Hf}_{\mathrm{CHUR}(0)}=0.282772 ;{ }^{176} \mathrm{Lu} /{ }^{177} \mathrm{Hf}_{\mathrm{CHUR}}=0.0332 ;{ }^{176} \mathrm{Hf} /{ }^{177} \mathrm{Hf}_{\mathrm{CC}}=0.015$.

sample 09AL66 that have the lowest abundances of total REE and positive Eu anomaly (fig. 6A), which is likely attributed to apatite fractionation and feldspar accumulation. In the primitive mantle-normalized spidergram, the granites show strong enrichment in $\mathrm{Th}$, but pronounced negative anomalies in $\mathrm{Nb}, \mathrm{Ta}, \mathrm{P}$ and Ti relative to the neighbor elements (fig. 6B).

Nine samples were selected for whole rock Sm-Nd isotope analyses. The Dabusushan and Naimumaodao two-mica granites have $\varepsilon_{\mathrm{Nd}}(\mathrm{t})$ values ranging from -10.1 to -4.5 and -5.8 to -5.3 , corresponding to two-stage $\mathrm{Nd}$ mode ages $\left(\mathrm{T}_{2 \mathrm{DM}}\right)$ of 2.38 to $1.93 \mathrm{Ga}$ and 2.05 to $2.01 \mathrm{Ga}$ (fig. 7), respectively.

The Gelintaishan amphibolitic gneiss. - Two amphibolitic gneisses samples from the Gelintaishan were analyzed for major and trace elements. They show low values in $\mathrm{SiO}_{2}$ (48.8-52.5 wt.\%), $\mathrm{TiO}_{2}$ (0.54-0.85 wt.\%), $\mathrm{K}_{2} \mathrm{O}$ (2.2-3.1 wt.\%), $\mathrm{Na}_{2} \mathrm{O}$ (0.37-0.39 wt.\%) and $\mathrm{Fe}_{2} \mathrm{O}_{3}{ }^{\mathrm{T}}$ (4.1-4.9 wt.\%), high values in $\mathrm{MgO}$ (13.0-13.3 wt.\%) and $\mathrm{CaO}$ (13.5-19.0 wt. \%), and variable $\mathrm{Al}_{2} \mathrm{O}_{3}$ values (7.7-14.4 wt.\%). They have similar REE partition patterns showing LREE enrichment and moderate negative Eu anomalies (fig. 6C). In the primitive mantle-normalized spidergram, these two gneisses show strong enrichment in $\mathrm{Th}$, but pronounced negative anomalies in $\mathrm{Nb}$, Ta, $\mathrm{P}$ and $\mathrm{Ti}$ relative to the neighbor elements (fig. 6D).

The characteristics of high $\mathrm{CaO}$ and $\mathrm{MgO}$ but extreme depletion in $\mathrm{Cr}, \mathrm{Ni}$ and $\mathrm{Fe}_{2} \mathrm{O}_{3}{ }^{\mathrm{T}}$ of the amphibolitic gneisses are different from those of igneous basaltic rocks 

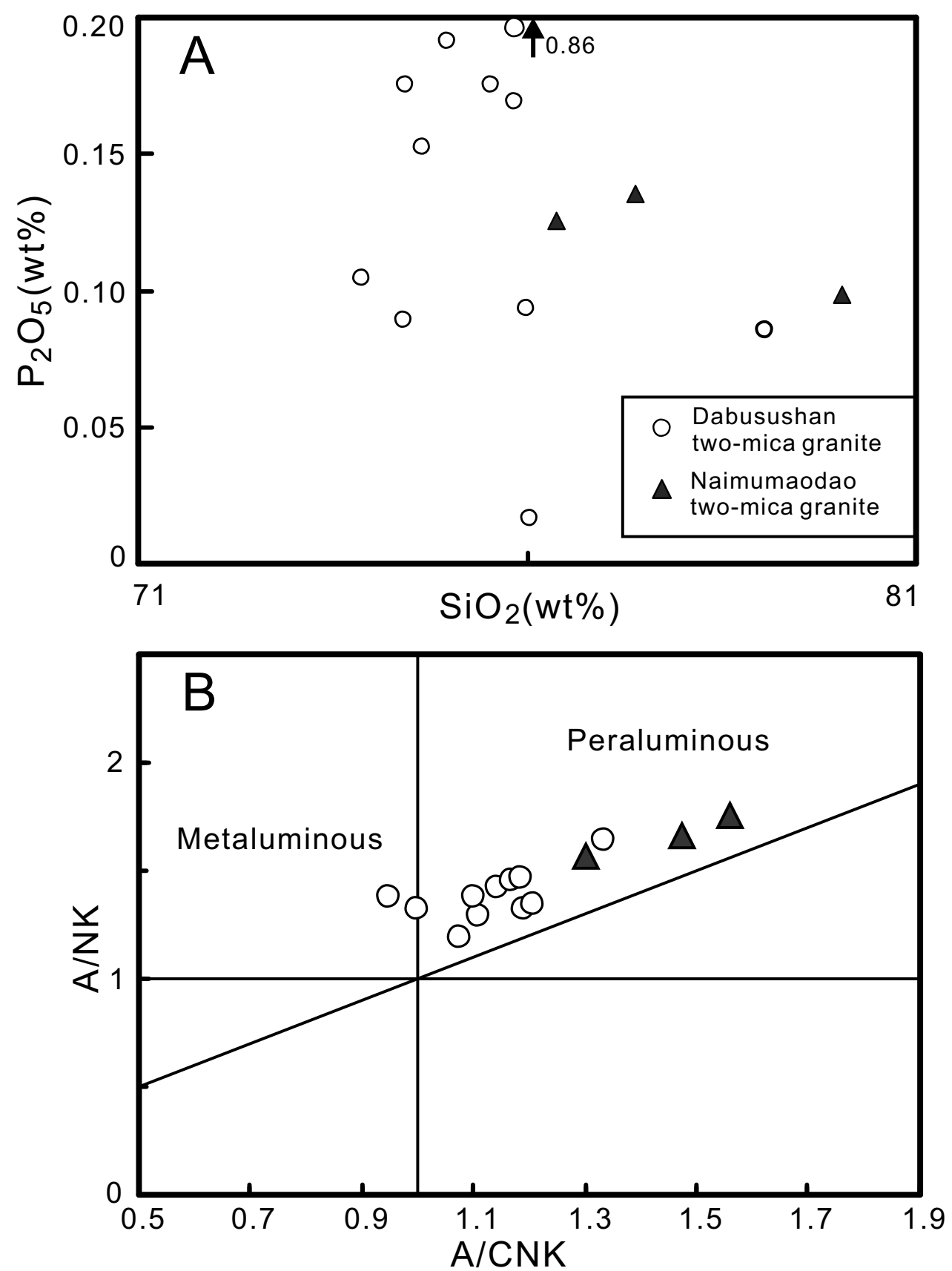

Fig. 5. Plots of (A) $\mathrm{SiO}_{2}$ vs. $\mathrm{P}_{2} \mathrm{O}_{5}$ and (B) A/CNK vs. A/NK for the Neoproterozoic granites.

(Rollinson, 1993). On the $\mathrm{TiO}_{2}$ versus $\mathrm{MnO}$ diagram of Mirsa (1971), the two samples plot into the sediments field (fig. 8), and they have negative DF3 values (table 1), indicative of sedimentary origins (Shaw, 1972). This chemical discrimination is consistent with their intercalation with other metasedimentary rocks in the field.

\section{Zircon U-Pb Ages}

The Dabusushan two-mica granite (09AL53 and 09AL62).-Zircon grains separated from sample 09AL53 are mostly euhedral to subhedral, with lengths of $\sim 100$ to 250 $\mu \mathrm{m}$, and length to width ratios of 2:1 to 4:1. Most grains show oscillatory zoning under 

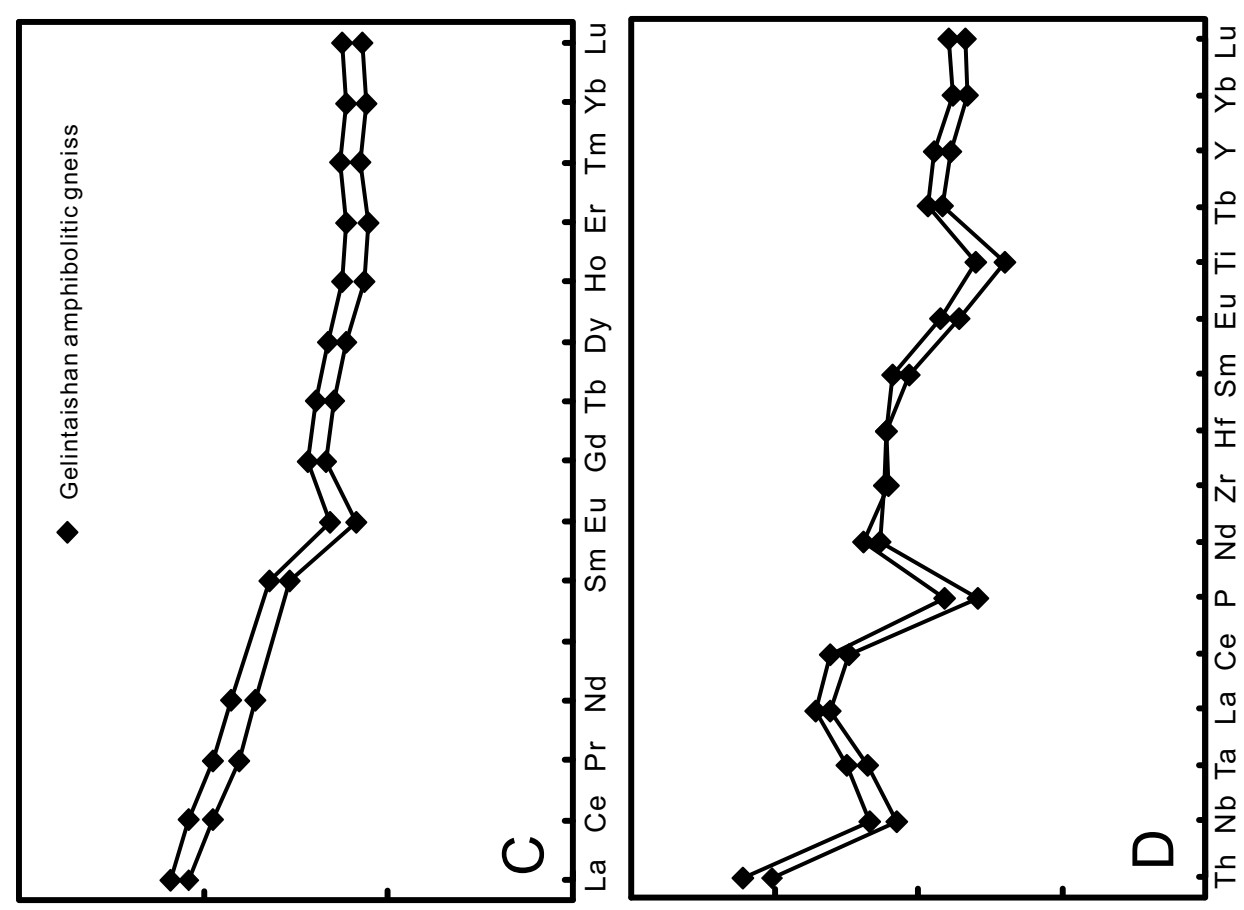

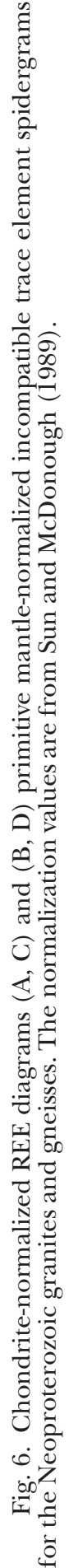
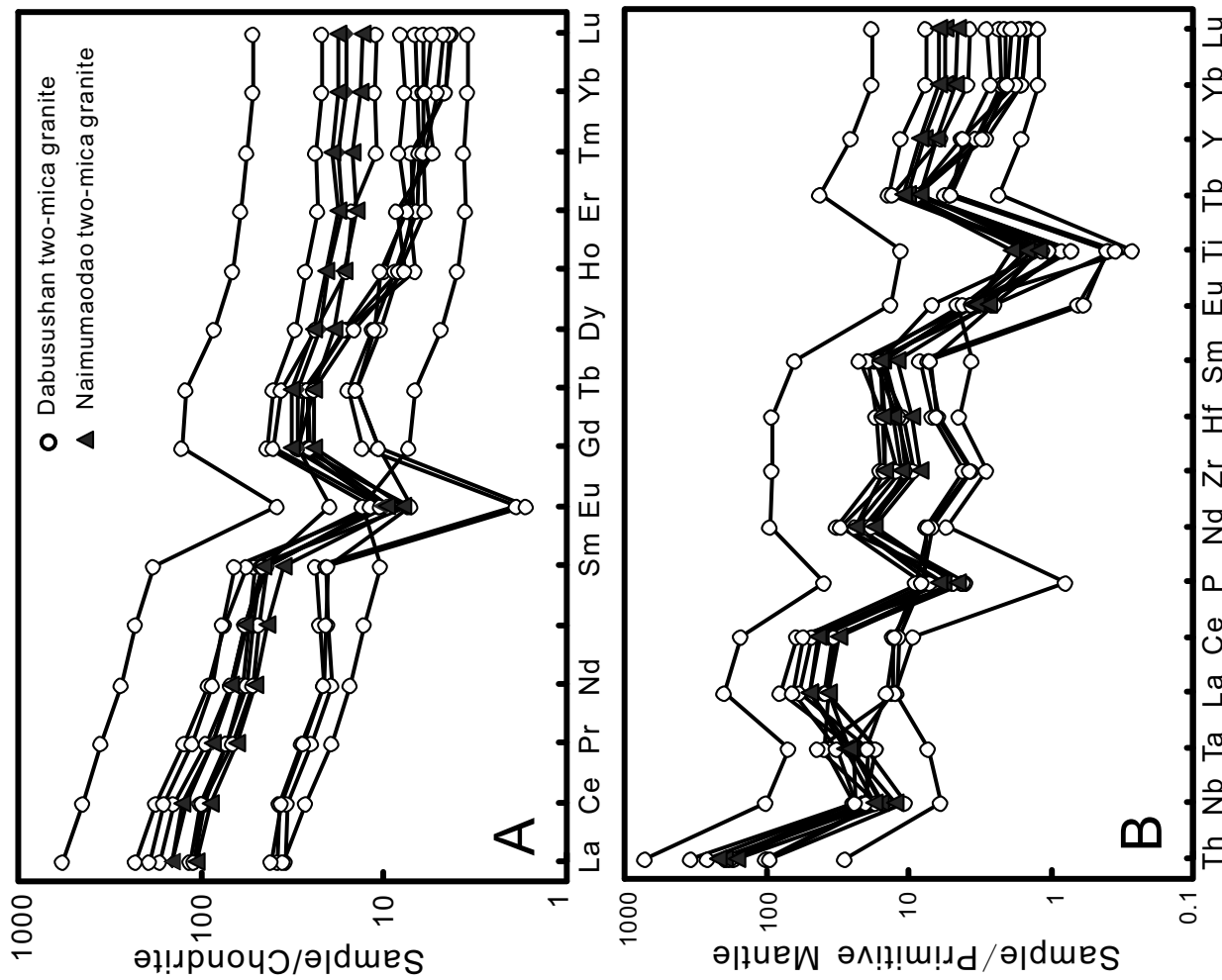


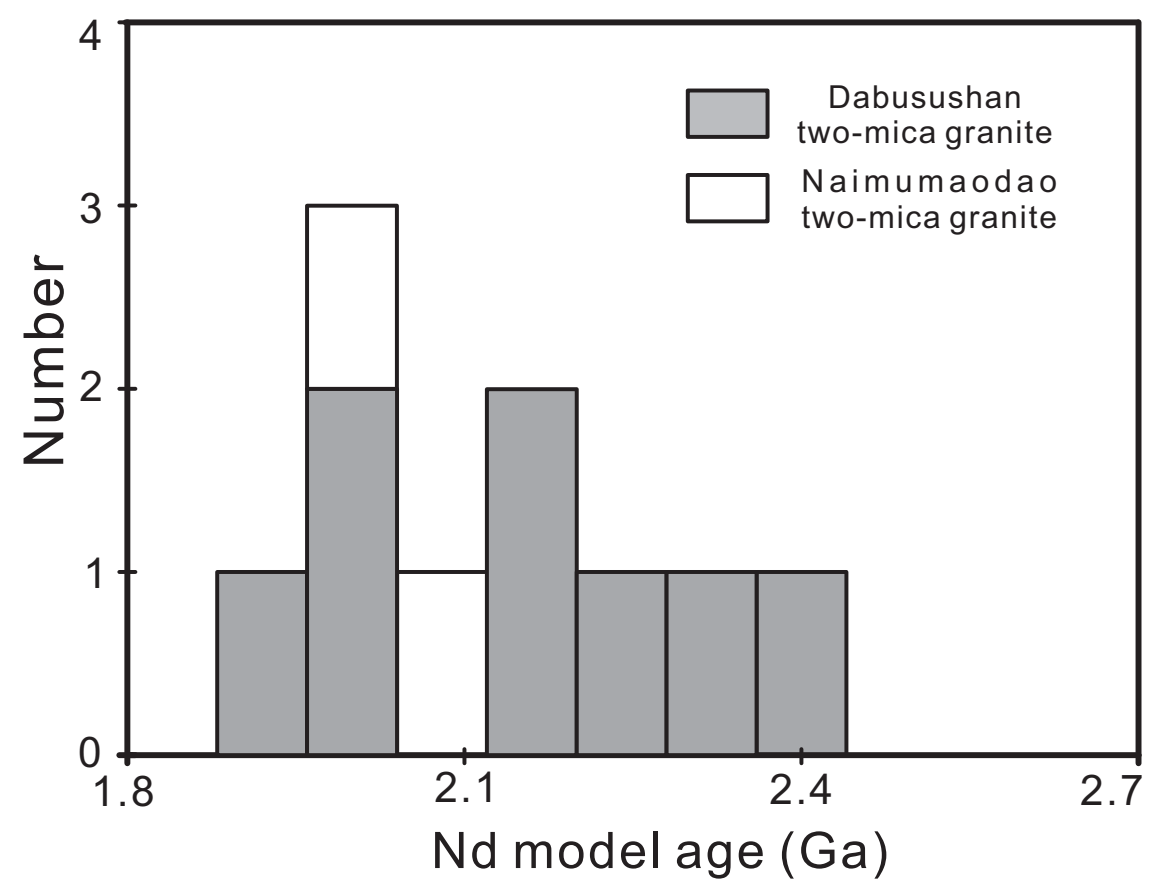

Fig. 7. Whole rock Nd model ages for the Neoproterozoic granites.

CL (fig. 9). Nineteen analyses were obtained from 18 grains, yielding $\mathrm{U}$ and Th contents of 74 to $630 \mathrm{ppm}$ and 18 to $170 \mathrm{ppm}$, respectively, with Th/U ratios of 0.07 to 1.62 (mostly of $0.40-1.16$ ). Sixteen analyses are concordant with $\mathrm{U} / \mathrm{Pb}$ ratios within

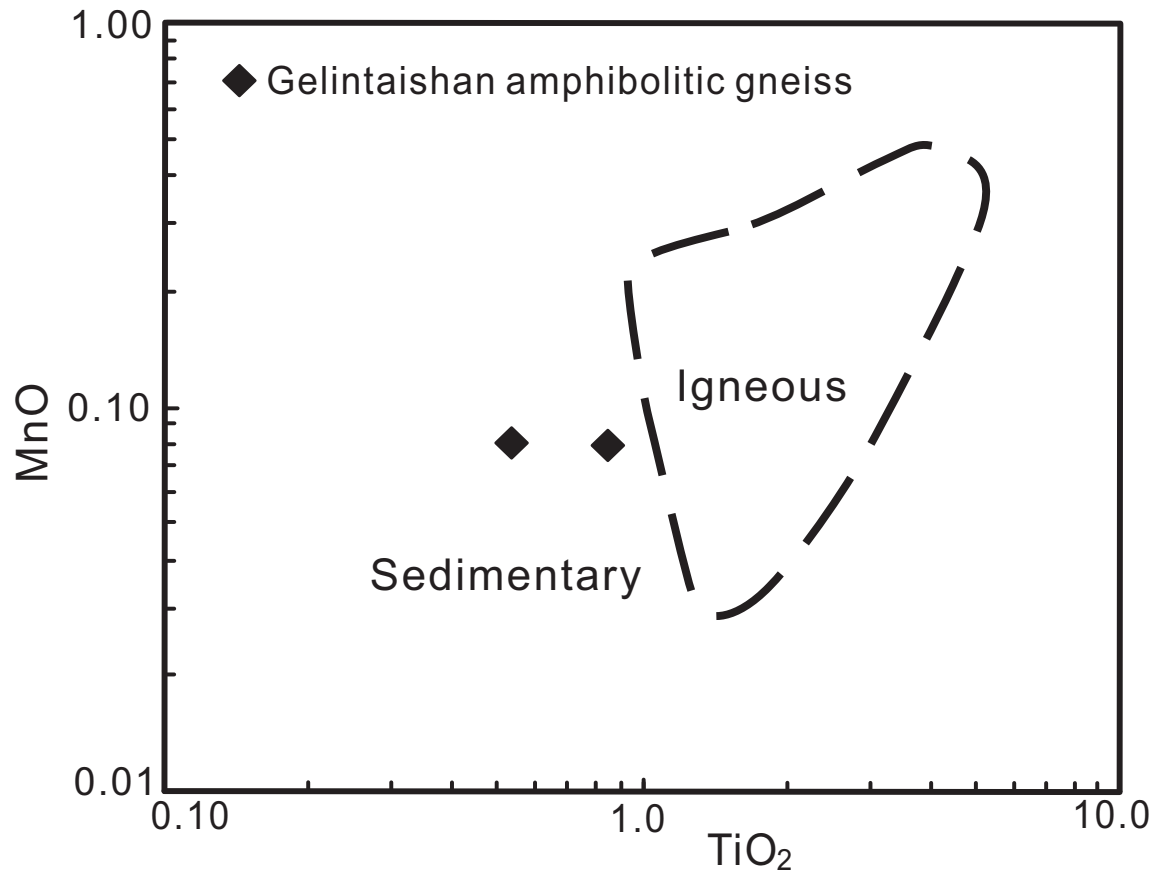

Fig. 8. Discrimination diagram of $\mathrm{MnO}$ vs. $\mathrm{TiO}_{2}$ (Misra, 1971) for distinguishing meta-igneous from metasedimentary rocks. 

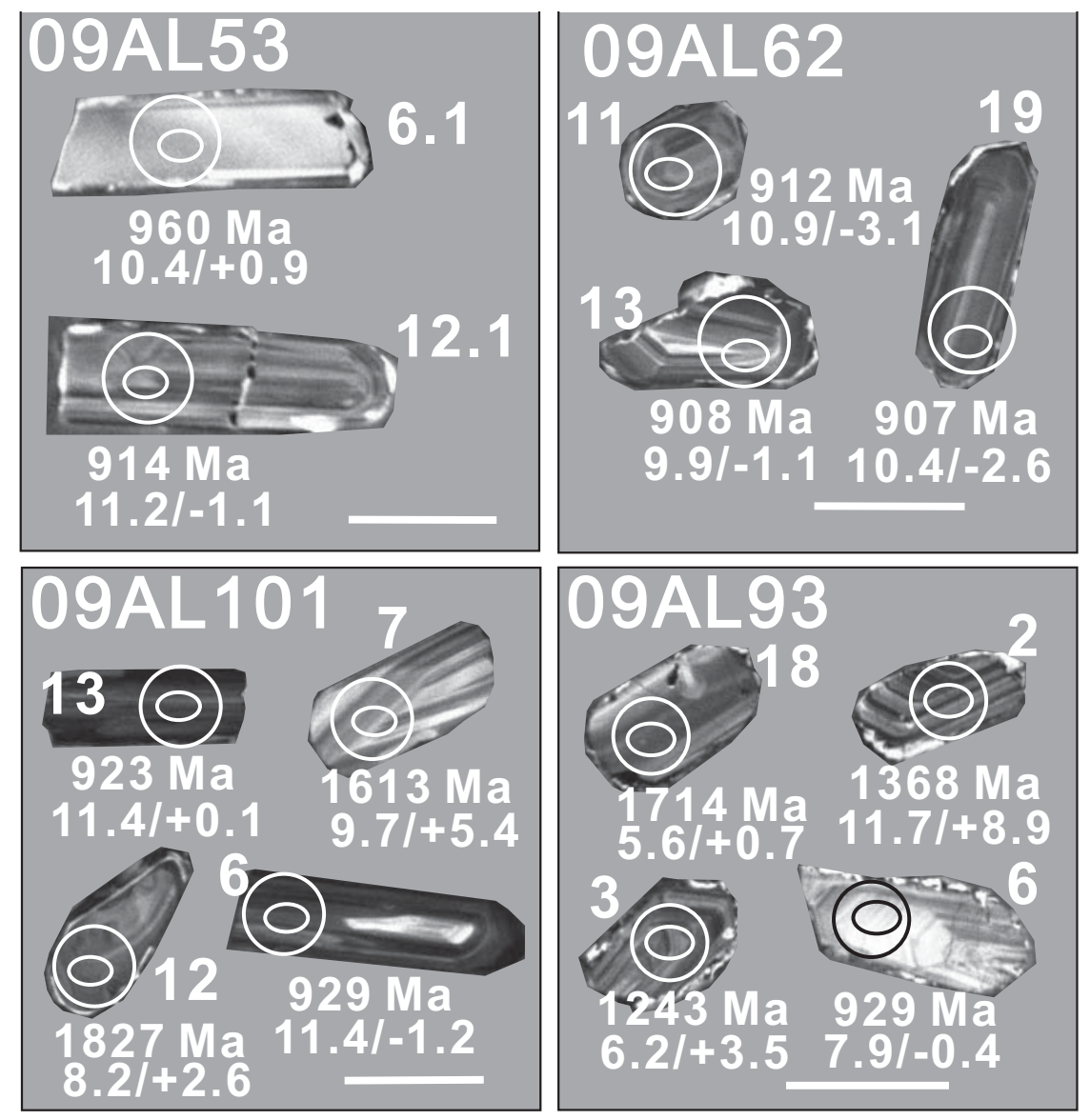

Fig. 9. Cathodoluminescence images of representative zircons for in situ analyses of U-Pb and/or Hf-O isotopes. Small ellipses indicate the SIMS analytical spots for U-Pb and/or O isotopes, and large circles denote the LA-MC-ICPMS analytical spots for Lu-Hf isotopes. Numbers near the analysis spots are the U-Pb ages $(\mathrm{Ma})$ and $\varepsilon_{\mathrm{Hf}}(\mathrm{t}) / \delta^{18} \mathrm{O}$ values. The white scale bars are $100 \mu \mathrm{m}$ long.

analytical errors (fig. 10A), yielding a weighted mean ${ }^{206} \mathrm{~Pb} /{ }^{238} \mathrm{U}$ age of $914 \pm 6 \mathrm{Ma}$ (MSWD $=0.23,95 \%$ confidence interval), which is interpreted as the crystallization age of this sample. Two analyses (spots 1.2 and 4.1) show slightly younger ages than the main population, probably due to radiogenic $\mathrm{Pb}$ loss. Spot 6.1 has a ${ }^{206} \mathrm{~Pb} /{ }^{238} \mathrm{U}$ age of $960 \pm 13 \mathrm{Ma}$, which is considered as the formation age of a xenocryst.

Most zircon grains from sample 09AL62 are euhedral to subhedral, with lengths of $\sim 100$ to $250 \mu \mathrm{m}$, and length to width ratios of 2:1 to 3:1. Most grains show oscillatory zoning under CL (fig. 9). Twenty analyses were obtained from 20 grains, yielding U and Th contents of 83 to $426 \mathrm{ppm}$ and 19 to $206 \mathrm{ppm}$, respectively, with Th/U ratios of 0.14 to 1.33 . Among them, fourteen analyses have concordant $\mathrm{U}-\mathrm{Pb}$ ages within analytical errors (fig. 10B), yielding a weighted mean ${ }^{206} \mathrm{~Pb} /{ }^{238} \mathrm{U}$ age of $909 \pm 7 \mathrm{Ma}$ (MSWD $=0.54,95 \%$ confidence interval), which is interpreted as the crystallization age of this sample. The remaining six analyses show slightly younger ages than the main population, probably due to radiogenic $\mathrm{Pb}$ loss.

The Naimumaodao two-mica granite (09AL101). - Most zircon grains from this sample are euhedral to subhedral, with lengths of $\sim 100$ to $250 \mu \mathrm{m}$ and length to width ratios of $\sim 2: 1$, and show oscillatory zoning under CL (fig. 9). Twenty-two analyses were obtained from 22 grains, yielding $\mathrm{U}$ and Th contents of 95 to $470 \mathrm{ppm}$ and 32 to 197 $\mathrm{ppm}$, respectively, with $\mathrm{Th} / \mathrm{U}$ ratios of 0.09 to 0.84 . Among them, seventeen analyses have concordant $\mathrm{U}-\mathrm{Pb}$ ages within analytical errors, yielding a weighted mean ${ }^{206} \mathrm{~Pb} /$ 

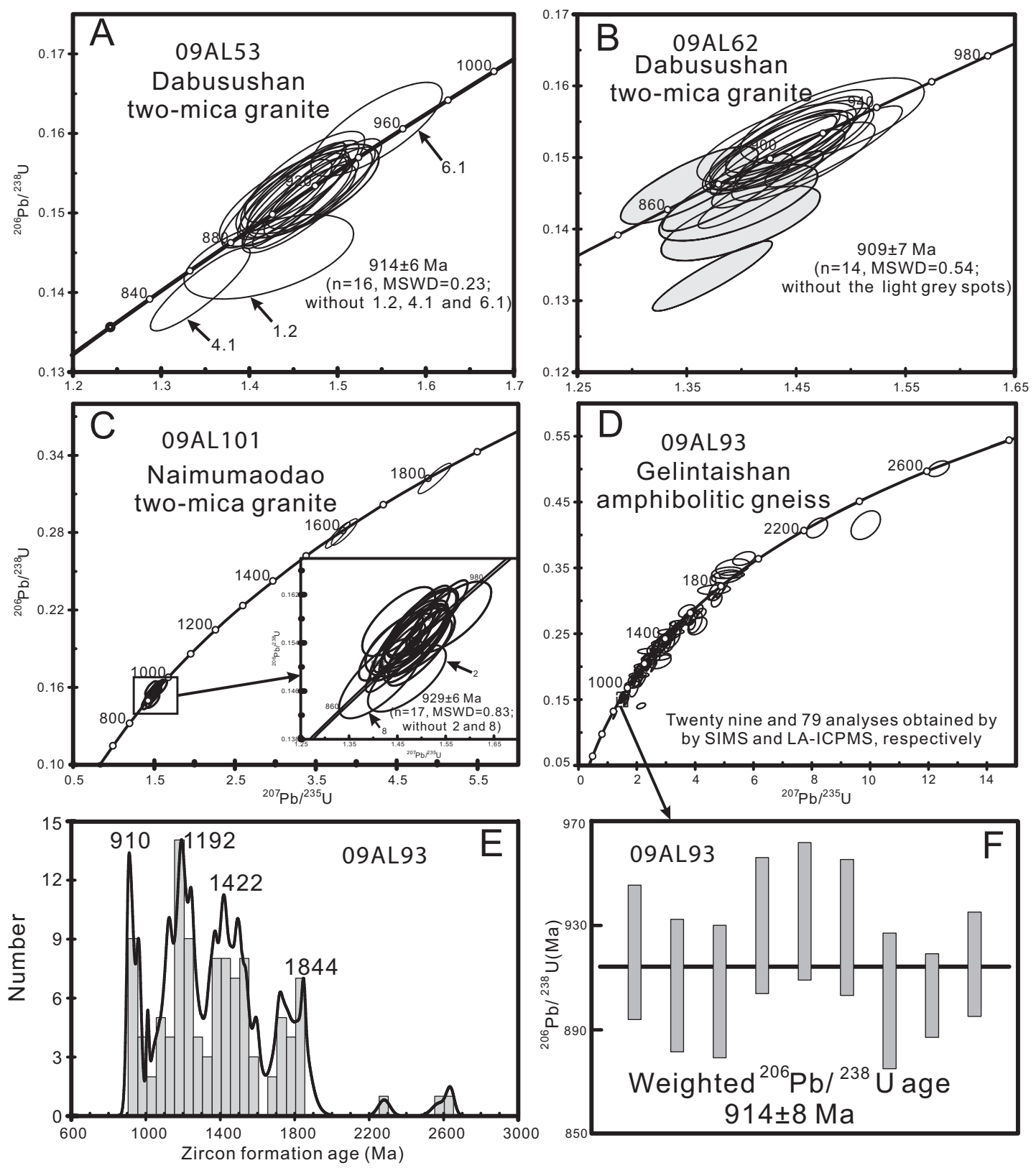

Fig. 10. In situ U-Pb dating results for the Neoproterozoic rocks. (A) two-mica granite from Dabusushan pluton (09AL53), (B) two-mica granite from Dabusushan pluton (09AL62), (C) two-mica granite from Naimumaodao pluton (09AL101), (D) Gelintaishan amphibolitic gneiss, (E) Probability plots of zircon formation age from 09AL93, (F) the youngest weighted age from 09AL93. Data-point error ellipses are $2 \sigma$.

${ }^{238} \mathrm{U}$ age of $929 \pm 6 \mathrm{Ma}(\mathrm{MSWD}=0.83,95 \%$ confidence interval) (fig. 10C), which is interpreted as the crystallization age of this sample. Spots 7, 11 and 12 yielded significantly old ${ }^{207} \mathrm{~Pb} /{ }^{206} \mathrm{~Pb}$ ages of $1613 \mathrm{Ma}, 1590 \mathrm{Ma}$ and $1827 \mathrm{Ma}$, respectively, which are interpreted to be the formation age of xenocrysts. The remaining two analyses (spots 2 and 8 ) show slightly younger ages than the main population, probably due to radiogenic $\mathrm{Pb}$ loss.

The Gelintaishan amphibolitic gneiss (O9AL93). - Zircon grains separated from this sample have lengths of $\sim 50$ to $100 \mu \mathrm{m}$ and length to width ratios of $\sim 2: 1$. The crystal morphologies vary from euhedral, stubby and sub-rounded, and most zircons show 
oscillatory zones under CL (fig. 9). Twenty-nine and 79 analyses were obtained from 108 grains by SIMS and LA-ICPMS technique, respectively, yielding $\mathrm{U}$ and Th contents of 20 to $675 \mathrm{ppm}$ and 40 to $706 \mathrm{ppm}$, respectively, with Th/U ratios of 0.08 to 2.14.

$\mathrm{On}$ the U-Pb Concordia diagram, all but two analyses plot at or near the Concordia curve (fig. 10D). Three analyses are older than $2.0 \mathrm{Ga}$, and the remaining 105 analyses yield ${ }^{207} \mathrm{~Pb} /{ }^{206} \mathrm{~Pb}$ ages between 1844 and $901 \mathrm{Ma}$, with four major age peaks at $\sim 0.91 \mathrm{Ga}, \sim 1.19 \mathrm{Ga}, \sim 1.42 \mathrm{Ga}$ and $\sim 1.84 \mathrm{Ga}$ (fig. $10 \mathrm{E}$ ). Nine youngest analyses have concordant $\mathrm{U}-\mathrm{Pb}$ ages within analytical errors, yielding a weighted ${ }^{206} \mathrm{~Pb} /{ }^{238} \mathrm{U}$ age of $914 \pm 8 \mathrm{Ma}$ (MSWD $=1.17,95 \%$ confidence interval) (fig. $10 \mathrm{~F}$ ). These zircons have $\mathrm{Th} / \mathrm{U}<1$, consistent with zircons crystallized from felsic melts (Heaman and others, 1990). Thus, they are most likely detritus in origin, and their age of $914 \pm 8$ Ma provides a maximum depositional age of the Deerhetongte Formation.

\section{Zircon Hf-O Isotopes}

The Dabusushan and Naimumaodao granites. - In situ LA-MC-ICPMS Lu-Hf isotopic analyses were conducted on the zircon grains that were previously analyzed for U-Pb and $\mathrm{O}$ isotopes by SIMS. The magmatic zircons have age-corrected $\varepsilon_{\mathrm{Hf}}(\mathrm{t})$ values ranging from -3.4 to $+3.8,-6.2$ to +0.8 and -2.9 to +0.3 , corresponding to two-stage zircon Hf model ages $\left(\mathrm{T}_{\mathrm{DM}}{ }^{\mathrm{C}}\right.$ ) of 2.01 to $1.55 \mathrm{Ga}, 2.18$ to $1.74 \mathrm{Ga}$ and 1.99 to $1.79 \mathrm{Ga}$, for samples 09AL53 and 09AL62 from the Dabusushan pluton and sample 09AL101 from the Naimumaodao pluton, respectively (table 4).

The measured $\delta^{18} \mathrm{O}$ values for magmatic zircons from these Neoproterozoic granites show a wide range of 8.2 to 12.1 permil, 9.2 to 12.1 permil, and 8.6 to 12.1 permil for samples 09AL53, 09AL62 and 09AL101, respectively. All the measured $\delta^{18} \mathrm{O}$ values from these three samples do not form normal Gaussian distributions (fig. 11), indicating their heterogeneous oxygen isotopic features.

The Gelintaishan amphibolitic gneiss (09AL93). - Twenty-nine in situ Lu-Hf isotopic analyses for sample 09AL93 yield the age-corrected $\varepsilon_{\mathrm{Hf}}(\mathrm{t})$ values ranging from -5.3 to +10.4 , and the two-stage zircon $\mathrm{Hf}$ model ages $\left(\mathrm{T}_{\mathrm{DM}}{ }^{\mathrm{C}}\right)$ ranging from 2.45 to 1.29 $\mathrm{Ga}$ (table 4). Their measured $\delta^{18} \mathrm{O}$ values show a wide range of 5.2 to 11.7 permil. Five of 6 zircons dated at $\sim 914 \mathrm{Ma}$ have $\delta^{18} \mathrm{O}$ values of 7.3 to 7.9 permil and the age-corrected $\varepsilon_{\mathrm{Hf}}(\mathrm{t})$ values of -4.0 to +2.7 , and other one ( 10.1 permil and $\varepsilon_{\mathrm{Hf}}(\mathrm{t})=-1.1$. Overall, these 6 zircons have two-stage zircon Hf model ages $\left(\mathrm{T}_{\mathrm{DM}}{ }^{\mathrm{C}}\right)$ ranging from 2.04 to $1.64 \mathrm{Ga}$.

\section{DISCUSSIONS}

\section{Granite Classification and Petrogenesis}

As aforementioned, classification of the Neoproterozoic granites in the Alxa Block is controversial. The Dabusushan and Naimumaodao granites were previously classified as S-type granites based on their peraluminous characteristics (Geng and others, 2002), such as $>1$ percent normative corundum, but recently re-classified as A-type granites based on other trace element characteristics (Geng and Zhou, 2011). It is noteworthy that these granites are highly evolved, with $\mathrm{SiO}_{2}=74$ to 80 weight percent, thus, proper classification based on mineralogy and chemical compositions is usually ambiguous (for example, Li and others, 2007). On the other hand, apatite shows different solubility in the strongly peraluminous S-type and metaluminous to weakly peraluminous I- and A-type granitic melts. $\mathrm{P}_{2} \mathrm{O}_{5}$ contents in the I- and A-type granites decrease with increasing $\mathrm{SiO}_{2}$, whilst $\mathrm{P}_{2} \mathrm{O}_{5}$ contents in S-type granites are either roughly constant or slightly increase with increasing $\mathrm{SiO}_{2}$ contents because apatite is generally under-saturated in strongly peraluminous melts due to its high solubility (for example, Chappell, 1999; Li and others, 2007; Bonin, 2007). The Dabusushan and Naimumaodao granites have high $\mathrm{P}_{2} \mathrm{O}_{5}$ abundance (mostly between 0.09 wt. $\%$ and 

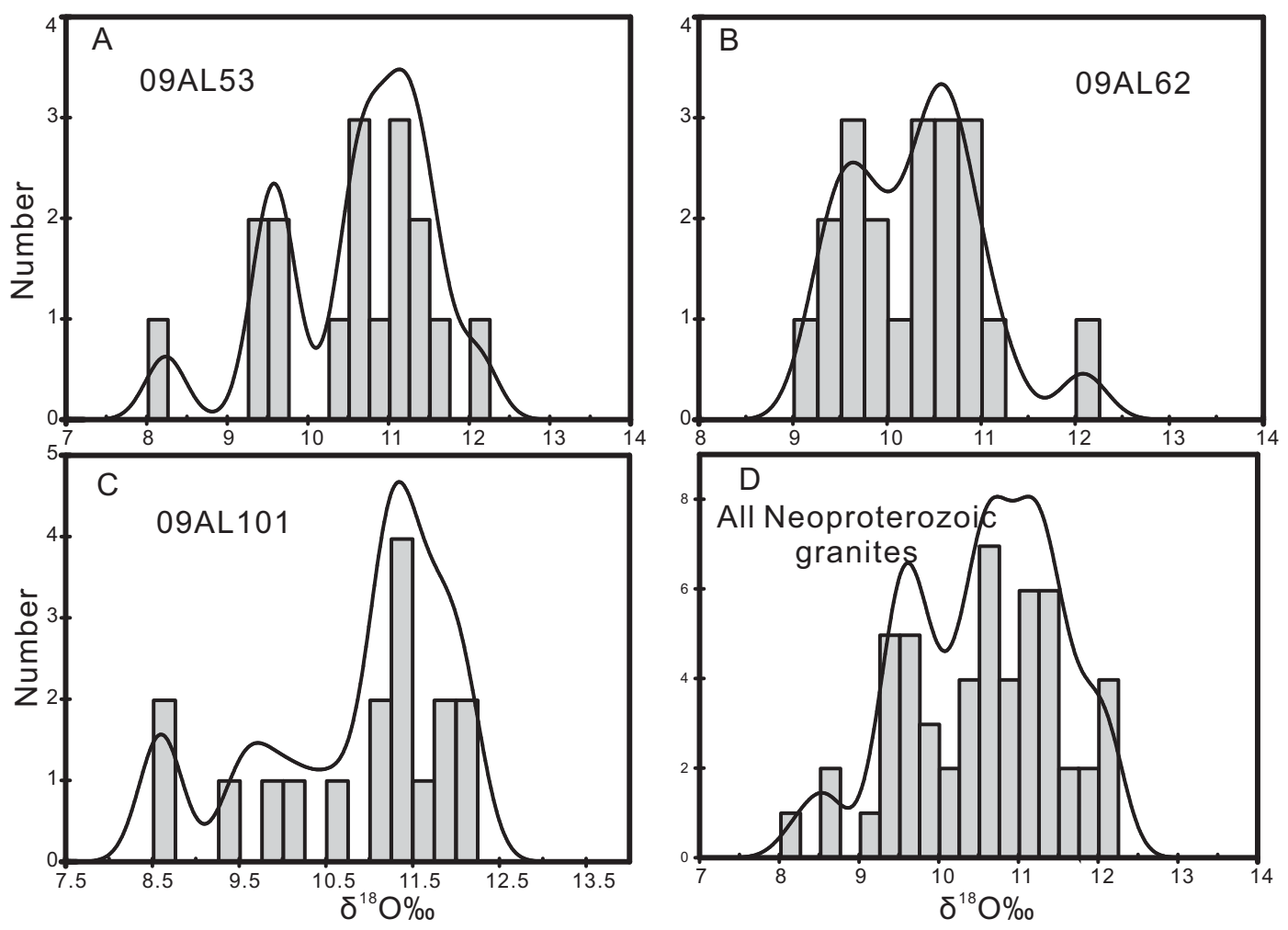

Fig. 11. Probability plots of zircon $\delta^{18} \mathrm{O}$ values from the Neoproterozoic granites.

0.86 wt.\%) at $\mathrm{SiO}_{2}$ content even up to 80 weight percent (table 1, fig. 5A), which is different in comparison to the $\mathrm{P}_{2} \mathrm{O}_{5}$ abundance of highly evolved peraluminous I- and A-type granites, but similar to those of the S-type granites in the South China and Lachlan Fold Belt in Australia (Chappell, 1999; Li and others, 2007). Moreover, these rocks have high zircon $\delta^{18} \mathrm{O}$ values of $c a$. 8.2 to 12.1 permil, corresponding to the calculated magmatic $\delta^{18} \mathrm{O}$ values of $c a$. 10.5 to 14.3 permil based on the relationship between zircon and whole-rock $\delta^{18} \mathrm{O}$ values (Valley and others, 2005). These $\delta^{18} \mathrm{O}$ values are comparable with those $\left(\delta^{18} \mathrm{O}>10 \%\right.$ ) of most S-type granites, but higher than those $\left(\delta^{18} \mathrm{O}=6-10 \%\right.$ ) of I-type granites (for example, O'Neil and Chappell, 1977). Xenocryst zircons are common in these samples (this study and Geng and Zhou, 2010), similar to most S-type granites (for example, Keay and others, 1999). All these lines of evidence indicate that these Neoproterozoic granites can be classified as S-type granites. This is consistent with melts derived from metagraywackes or metapelites (fig. 12). Although Geng and Zhou (2010) noted that some granites show high $10000 \mathrm{Ga} / \mathrm{Al}$ values $(>2.6)$, almost all of them have low $\mathrm{FeO}^{\mathrm{T}} / \mathrm{MgO}$ values $(<10)$ (fig. $13)$ and low $(\mathrm{Zr}+\mathrm{Nb}+\mathrm{Ce}+\mathrm{Y})$ contents $(<350 \mathrm{ppm})$ (this study and Geng and Zhou, 2010). Thus, they should not be classified as A-type granites (Bonin, 2007).

In recent years, some researchers reported that a few S-type granites contain mantle-derived materials (for example, Healy and others, 2004; Zhu and others, 2009) and/or intracrustal components based on in situ zircon Hf-O isotopes (Appleby and others, 2010). The granites in this study have zircon $\delta^{18} \mathrm{O}>8$ permil, obviously higher than mantle-derived materials with zircon $\delta^{18} \mathrm{O}=5.3$ permil, suggesting these rocks were most likely sourced from supracrustal rocks without any detectable mantle components. Each single sample shows a wide variation in the value of zircon $\delta^{18} \mathrm{O}$ 


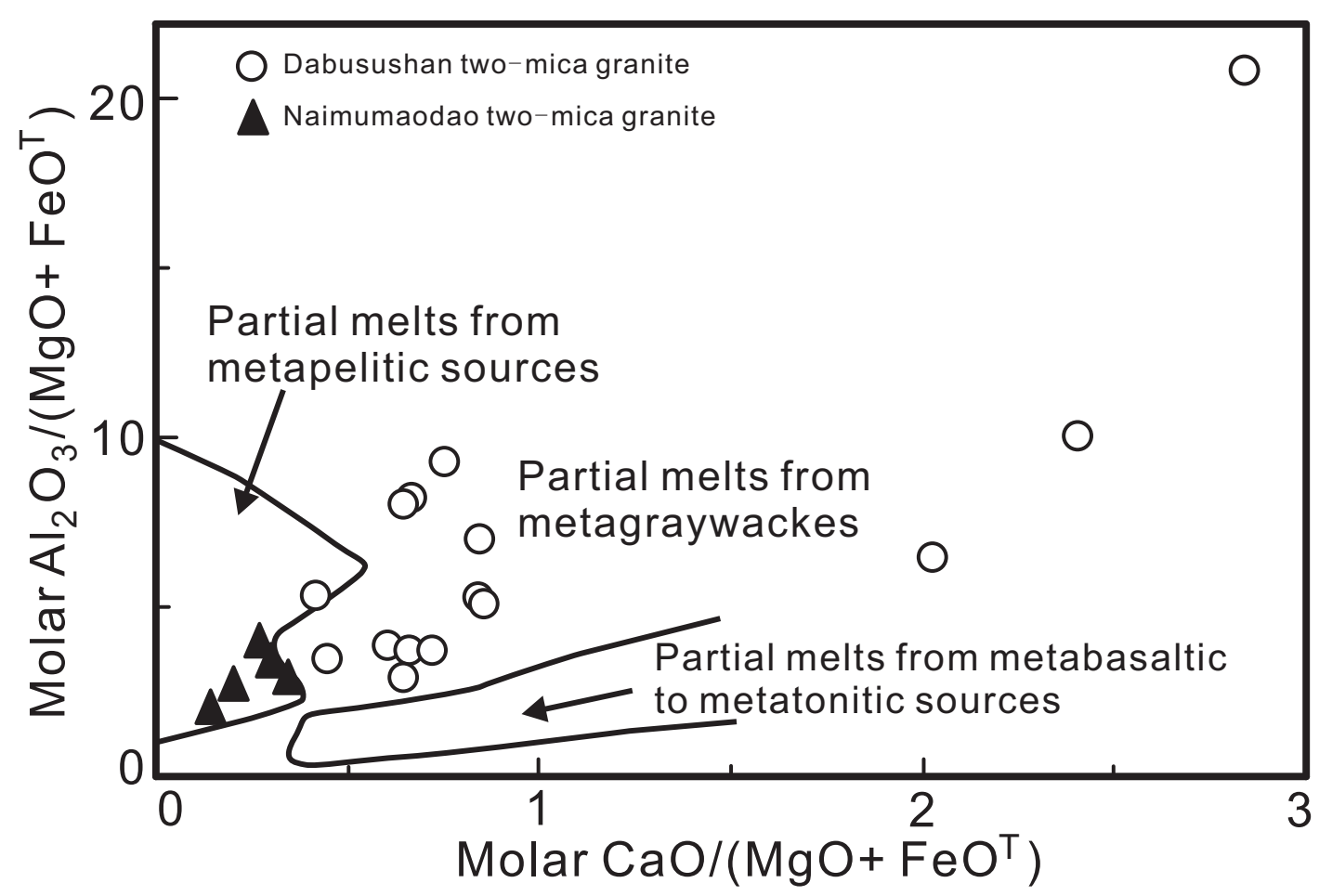

Fig. 12. Molar $\mathrm{Al}_{2} \mathrm{O}_{3} /\left(\mathrm{MgO}+\mathrm{FeO}^{\mathrm{T}}\right)$ vs. molar $\mathrm{CaO} /\left(\mathrm{MgO}+\mathrm{FeO}^{\mathrm{T}}\right)$ for the Neoproterozoic granites (modified from Altherr and others, 2000), with six added data from Geng and Zhou (2011).

(about 3-4 $\delta$ units) (fig. 11), together with a large variation of zircon $\varepsilon_{\mathrm{Hf}}(\mathrm{t})$ values, especially for the Dabusushan granites (up to $10 \varepsilon$ units) (fig. 14). Such large O-Hf isotopic variations imply highly variable compositions of the granite sources. This interpretation is consistent with the highly variable whole-rock Nd isotopic compositions, such as the Dabusushan granites (fig. 7), and very large chemical variations on the diagram of $\mathrm{Al}_{2} \mathrm{O}_{3} /\left(\mathrm{MgO}+\mathrm{FeO}_{\mathrm{T}}\right)$ vs. $\mathrm{CaO} /\left(\mathrm{MgO}+\mathrm{FeO}_{\mathrm{T}}\right)$ (fig. 12). The zircon O-isotope data further suggest that there are at least two crustal sources involved in these rocks. While the high zircon $\delta^{18} \mathrm{O}$ value $(12.1 \%$ ) represents a supracrustal source, the low zircon $\delta^{18} \mathrm{O}$ value $(8.2 \%$ ) probably indicates an infracrustal component. This infracrustal component should account for only a small proportion, because there are only a few of zircons that have relatively low $\delta^{18} \mathrm{O}$ values $(8.2-8.6 \%$ ) in the granites. Furthermore, their $\mathrm{Nd}$ model ages $(c a .2 .4-1.9 \mathrm{Ga})$ suggest these granites were generated by melting of Paleoproterozoic materials with little, if any, involvement of Archean components.

\section{Tectonic Setting for the Neoproterozoic S-type Granites}

The Neoproterozoic granites in the Alxa Block were previously considered to have been formed either in a syn-collisional, or post-collisional setting (Geng and others, 2002; Geng and Zhou, 2011). It is noted that the S-type granites can also be formed in non-orogenic settings (for example, Li and others, 2003; Collins and Richards, 2008). For instance, the $c a .825$ to $820 \mathrm{Ma}$ S-type granites in South China are interpreted to be formed by remelting of supracrustal components related to mantle plume activities (for example, Li and others, 2003). This possibility, however, can be ruled out for the genesis of the Neoproterozoic S-type granites in the Alxa Block, because (1) the coeval basaltic rocks which are predominant in the large igneous provinces (LIPs) are not observed in this area, and (2) there is no evidence for existence of a $c a .930$ to $910 \mathrm{Ma}$ 


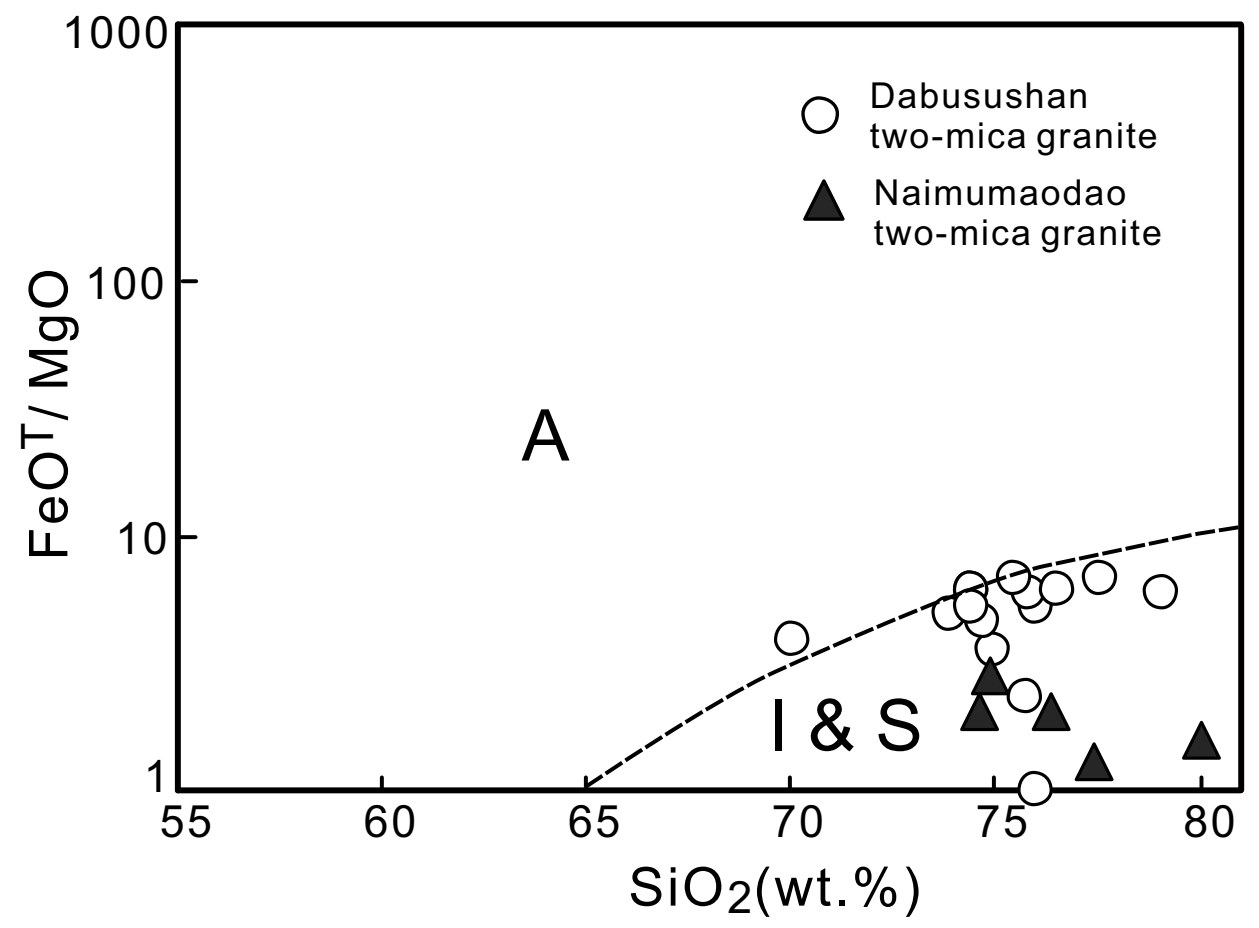

Fig. 13. Discrimination diagram of $\mathrm{FeO}^{\mathrm{T}} / \mathrm{MgO}$ vs. $\mathrm{SiO}_{2}$ for the Neoproterozoic granites (Eby, 1990), with six added data from Geng and Zhou (2011).

mantle plume or superplume in the Alxa Block or adjacent areas. Furthermore, these granites underwent strong deformation, especially the sub-solidus deformation, suggesting they were formed in a compressional environment (Paterson and others, 1989; Bouchez and others, 1992).

An alternative possibility is that these Neoproterozoic S-type granites were formed in the continental arc, such as that in the Lachlan Fold Belt in Australia (for example, Chappell and White, 1992). The S-type granites formed in this tectonic setting commonly contain some mantle-derived melts and/or intracrustal materials (for example, Healy and others, 2004; Zhu and others, 2009), or sometimes they are sourced from relatively young volcanogenic meta-sedimentary rocks (for example, Jeon and others, 2012). In contrast, the early Neoproterozoic S-type granites in the Alxa Block were produced by remelting of predominantly mature supracrustal materials. Coeval I-type granites, which are commonly considered to be produced in subduction zones (Barbarin, 1999), have not been recognized in the region. It is noted that five detrital zircons dated at $c a .914$ Ma from the amphibolite sample 09AL93 have $\delta^{18} \mathrm{O}$ value of 7.3 to 7.9 permil, which are consistent with those of zircon $\delta^{18} \mathrm{O}$ values for most I-type granites, but clearly lower than the zircon $\delta^{18} \mathrm{O}$ value of 8.2 to 12.1 permil for the studied Neoproterozoic S-type granites. These zircons are all rounded in shape (fig. 9), suggesting a long-distance transportation from their sources. Thus, until now, no evidence suggests that coeval, arc-related I-type granites exist in the Alxa Block, although the continental arc setting for the Neoproterozoic S-type granites cannot be entirely excluded.

Based on the available data, the Alxa Neoproterozoic S-type granites were most likely generated by remelting of dominant (meta)sedimentary rocks in an orogenesisrelated compressional environment. This interpretation is consistent with the regional angular unconformity between the metamorphosed Alxa Group and un-metamor- 


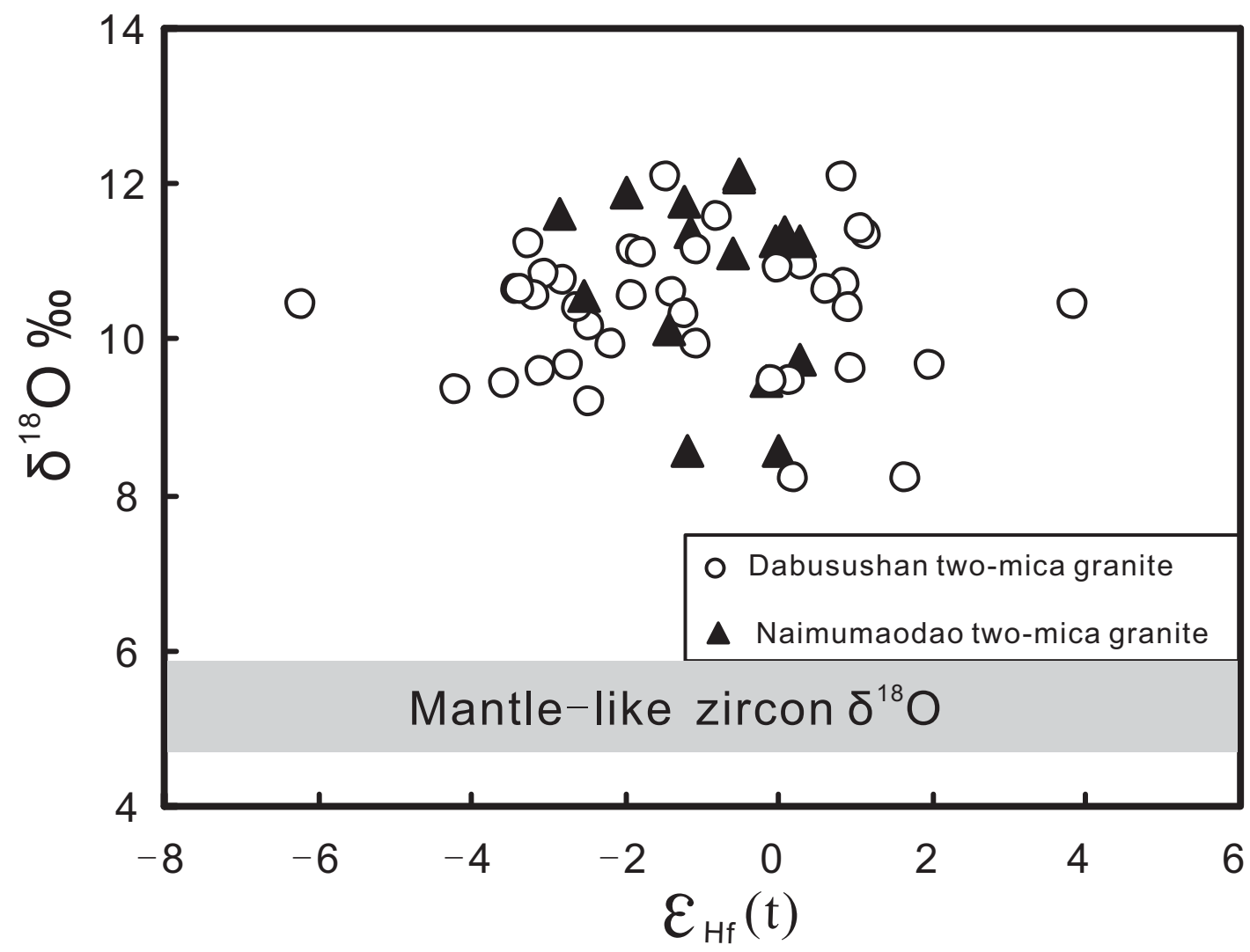

Fig. 14. Plot of $\varepsilon_{\mathrm{Hf}}(\mathrm{t})$ values vs. $\delta^{18} \mathrm{O}$ values for the magmatic zircons from the Neoproterozoic granites.

phosed Neoproterozoic sedimentary sequences (NMBGMR, 1991; Chen and others, 2004), although the age of this unconformity is not exactly defined.

\section{Implication for the Tectonic Affinity of Alxa Block}

The Alxa Block has long been thought as the westernmost part of NCC (Ren and others, 1987; Wu and others, 1998; Kusky and Li, 2003; Zhao and others, 2005), with the Archean basement rocks exposed in the eastern margin of the Alxa Block (NMBGMR, 1991). However, recent SIMS U-Pb zircon dating results indicate that both the Bayanwulashan and the Diebusige complexes, which were considered as the oldest rocks of the Alxa Block (NMBGMR, 1991), were formed in the Early Paleoproterozoic, not Archean (Dan and others, 2012b). Thus, we suggested that the Alxa Block is neither the western extension of the Archean Yinshan Block (Zhao and others, 2005; Zhao, 2009), nor part of the Late Paleoproterozoic Khondalite Belt (Dong and others, 2007; Geng and others, 2010) based on their discrepancy in the Paleoproterozoic geology (Dan and others, 2012b). However, the newly-identified $\sim 2.5$ Ga granitic gneisses in the western Alxa Block and $\sim 1.85$ Ga metamorphism in the Beidashan and Longshoushan complexes (Gong and others, 2011, 2012) imply that the Alxa Block might be a part of the NCC or adjacent to the NCC during assembly of the Columbia supercontinent. Thus, the relationship between the Alxa Block and the NCC during the Paleoproterozoic time awaits further investigations.

Our integrated $\mathrm{U}-\mathrm{Pb}$ and $\mathrm{Hf}-\mathrm{O}$ data in this study suggest that the Neoproterozoic geology in the Alxa Block is different from the NCC. The Neoproterozoic mafic dykes mostly formed at $c a .925$ to $900 \mathrm{Ma}$ in the central and eastern NCC (Gao and others, 2009; Peng and others, 2011a, 2011b; Wang and others, 2012a), which are considered 
as a part of a large igneous province (Peng and others, 2011a). A younger $c a .830 \mathrm{Ma}$ mafic magmatism, identified in the southern margin of the NCC, was also considered as the manifestation of continental break-up (Wang and others, 2011). Contrarily, the ca. 930 to 910 Ma S-type granites in the Alxa Block were most likely formed in an orogenesis-related compressional setting, clearly different from those rift-related $c a$. 925 to 900 Ma mafic dikes in central and eastern NCC.

There is a sharp contrast in the age patterns of detrital zircons from the Meso-Neoproterozoic metasedimentary rocks between the Alxa Block and the NCC. The detrital zircon ages from the Alxa Block are mostly around $\sim 1.85$ to $0.91 \mathrm{Ga}$ (only $4 \%$ of detrital zircons are older than $1.85 \mathrm{Ga}$ ), with peaks at $\sim 965 \mathrm{Ma}, \sim 1117 \mathrm{Ma}$, $\sim 1199 \mathrm{Ma}$ and $\sim 1433 \mathrm{Ma}$ (fig. 15C). In contrast, the detrital zircon ages from the NCG are mostly between $\sim 2.8 \mathrm{Ga}$ and $1.2 \mathrm{Ga}$, with age peaks at $\sim 2.7 \mathrm{Ga}, \sim 2.5 \mathrm{Ga}$ and $\sim 1.85$ Ga (for example, Hu and others, 2009; Wan and others, 2011; Sun and others, 2012). In the Zhuozishan area of western NCC, adjacent to the Alxa Block (fig. 2), the detrital zircon ages from the Mesoproterozoic-Cambrian sedimentary rocks are $\sim 2.8$ to $1.7 \mathrm{Ga}$, with peaks at $\sim 2.7 \mathrm{Ga}, \sim 1.95 \mathrm{Ga}, \sim 2.0$ to $2.1 \mathrm{Ga}$ and $\sim 2.37 \mathrm{Ga}$ (fig. 15A) (Darby and Gehrels, 2006). Thus, the detrital zircon population from the Meso-Neoproterozoic strata in the Alxa Block is clearly in contrast to the NCC. The small portion of $>1.85 \mathrm{Ga}$ detrital zircons in the Alxa Block suggests limited Paleoproterozoic rocks exposed in this block, implying that most of the Alxa Block could have been formed later than the Archean NCG.

Thus, the Alxa Block is likely a separated terrane from the NCC at least during the Late Precambrian time. The timing of the assemblage between the Alxa Block and NCG can be inferred from comparison of detrital zircon age patterns from the Mesoproterozoic-Early Paleozoic strata between the western margin of the NCC and the Alxa Block (Darby and Gehrels, 2006; Zhang and others, 2012). In the Zhuozishan area (fig. 2), all detrital zircon ages from the Mesoproterozoic-Cambrian Ordovician sedimentary rocks are older than $1.7 \mathrm{Ga}$ (fig. 15A), and the Ordovician sediments show two additional age peaks at $\sim 2.7 \mathrm{Ga}$ and $\sim 2.6 \mathrm{Ga}$ (fig. 4 in Darby and Gehrels, 2006), implying a different provenance. In contrast, the detrital zircons from the Helanshan Ordovician sediments are mostly between 1.2 and $0.9 \mathrm{Ga}$ (Zhang and others, 2012), with obvious age peak at $\sim 910 \mathrm{Ma}$ and $\sim 955 \mathrm{Ma}$ (fig. 15B). These results suggest that the provenances for the sedimentary rocks in the western margin of the NCG changed significantly in a short time between Cambrian and Ordovician. The detrital age pattern from the Helanshan Ordovician sediments, especially the predominantly ages between 1.2 and $0.9 \mathrm{Ga}$ (fig. 15B), is similar to that of the Meso-Neoproterozoic sedimentary rocks from the Alxa Block (fig. 15C). This similarity implies that the Ordovician sediments in the Helanshan area were probably derived from the Alxa Block. An alternative provenance for the Ordovician sediments in the Helanshan is likely the Central Qilian Block. However, the Paleozoic North Qilian arc, which was located between the Central Qilian Block and Alxa Block, would obstruct the Central Qilian Block as a major provenance (Zhang and Others, 2012). Thus, the Alxa Block and the NCC were probably amalgamated during the Early Paleozoic (Zhang and others, 2011, 2012). This suggestion is consistent with a regional angular unconformity between the Early and Late Paleozoic strata in the western Ordos Block of the NCG (Li and others, 2012).

Detrital zircon ages of this study and previous works (Li, 2006; Geng and Zhou, 2010) may place constraints on the affinity of the Alxa Block to other continents worldwide in the Early Neoproterozoic. Figure 16 presents a compilation of Precambrian detrital zircon ages for major continents and/or blocks, including the Alxa Block, Cathaysia and Yangtze blocks of South China, eastern Antarctic, southeastern Laurentia, western Baltic, northeastern Australia, western Amazonia, and Tarim Block. 

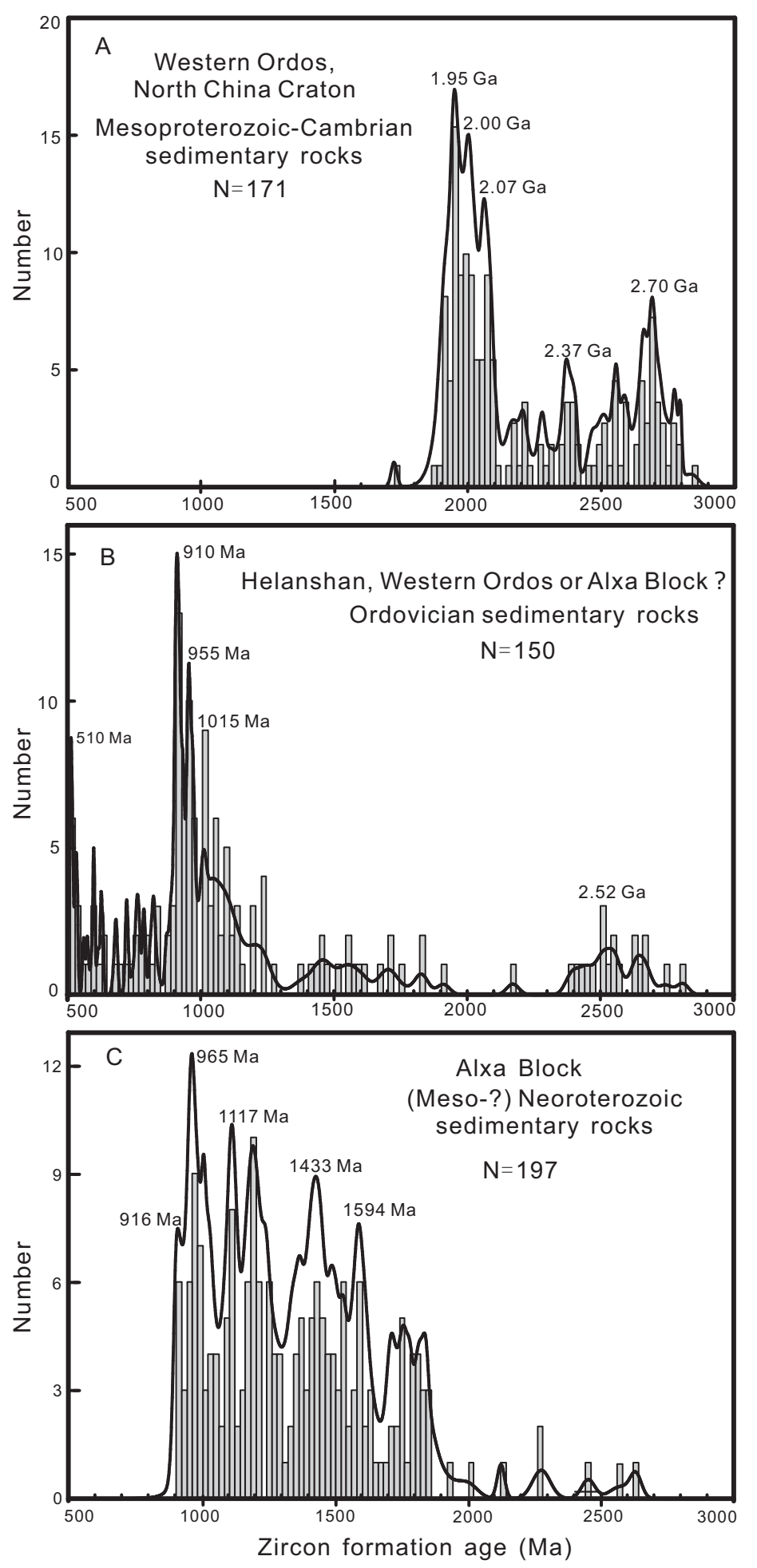

Fig. 15. Detrital zircon age patterns of sedimentary rocks from the Alxa Block and the western Ordos, North China Craton. (A) Proterozoic-Cambrian sediments from western Ordos (Barby and Gehrels, 2006), (B) Ordovician sediments from western Ordos (Zhang and others, 2012), (C) (Meso-?) Neoproterozoic sediments from the Alxa Block (including some xenocryst data from Neoproterozoic igneous rocks; Li, ms, 2006; Geng and others, 2007, 2010; this study). 


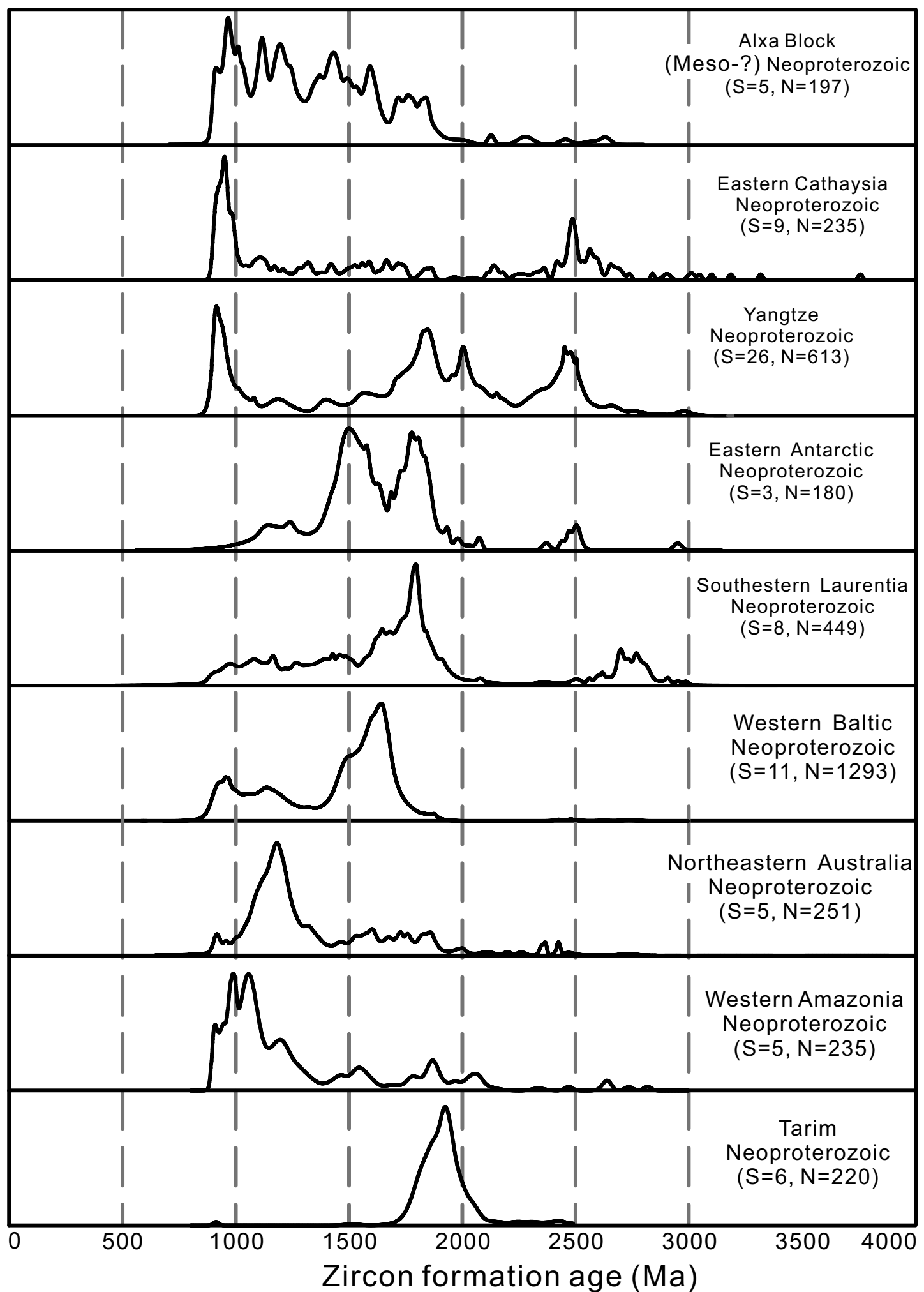

Fig. 16. Probability density plots for the detrital zircon ages older than $900 \mathrm{Ma}$ from the Neoproterozoic sedimentary samples from a number of continents and blocks worldwide. Data Sources: Alxa Block (including some xenocryst data from Neoproterozoic igneous rocks; Li, ms, 2006; Geng and others, 2007, 2010; this study); Eastern Cathaysia (Yu and others, 2008, 2010); Yangtz (Sun and others, 2009; Wang and others, 2010; Wang and others, 2012b); Eastern Antarctic (Goodge and others, 2004); Southeastern Laurentia (Cawood and others, 2003); Western Baltic (Be'eri-Shlevin and others, 2011); Northeastern Australia (Fergusson and others, 2001, 2007); Western Amazonia (Adams and others, 2008, 2011); Tarim (Shu and others, 2011; and Zhu and others, 2011). S and $\mathrm{N}$ are the number of samples and detrital zircon ages, respectively. Ages of $<1000 \mathrm{Ma}$ and $>1000 \mathrm{Ma}$ were calculated from their ${ }^{206} \mathrm{~Pb} /{ }^{238} \mathrm{U}$ and ${ }^{207} \mathrm{~Pb} /{ }^{206} \mathrm{~Pb}$ ratios, respectively. Ages with concordance $\geq 80 \%$ are used. 
The tectono-magmatic events younger than $900 \mathrm{Ma}$ are not discussed here as it is beyond the scope of this study. Figure 16 presents the detrital zircon age patterns for the aforementioned continents/blocks. There seems to be a similarity of detrital zircon age patterns between the Alxa Block and Cathaysia Block of South China (fig. 16 ), as both blocks exhibit a major age peak at $\sim 960$ Ma. Such similarity implies that the Alxa Block might be affinitive with the Cathaysia Block in Neoproterozoic time, or sediments in both blocks share similar sources. If this interpretation is correct, this conjectural orogen could be coeval with the $c a$. 1.0 to $0.9 \mathrm{Ga}$ Sibao Orogen in South China (Ye and others, 2007; Li and others, 2009b) and the 990 to $900 \mathrm{Ma}$ high-grade metamorphic events in both the Eastern Ghats Belt of India and the corresponding Rayner Province in East Antarctica (Mezger and Cosca, 1999; Boger and others, 2000; Fitzsimons, 2000; Kelly and others, 2002). Thus, the Alxa Block could have been involved in the assembly of Rodinia supercontinent (Li and others, 2008).

\section{CONCLUSIONS}

We draw the following conclusions based on our new geochronological and geochemical data:

(1) The Neoproterozoic granites in the Alxa Block are S-type granites formed at ca. 930 to $910 \mathrm{Ma}$. They are highly siliceous and were generated by remelting of predominantly metasedimentary rocks in an orogenesis-related compressional environment.

(2) The Alxa Block has a distinctive evolution from the North China Craton at least during the Neoproterozoic, suggesting that the Alxa Block is probably a separate terrane from the NCC during this time.

(3) Detrital zircon age data indicate that the Alxa Block is likely affinitive with the Cathaysia Block of South China in the Neoproterozoic time. Whereas, it appears akin with the NCC since Ordovician, suggesting that the amalgamation between the Alxa Block and the NCC might take place in the Early Paleozoic time.

\section{ACKNOWLEDGMENTS}

We thank Q. L. Li and G. Q. Tang for their help in SIMS analyses and Y. H. Yang for LA-MC-ICPMS analyses. Thoughtful and constructive comments by Dr. J. X. Zhang and an anonymous reviewer, and the editorial comments by Greg Shellnutt, substantially improved the manuscript. This study was financially supported by the National Natural Science Foundation of China (No. 41221002) and the research grant of State Key Laboratory of Geological Processes and Mineral Resources (grant No. GPMR201105). This is TIGeR (The Institute of Geoscience Research) publication No. 484 and contribution 343 from the ARC Center of Excellence for Core to Crust Fluid Systems (http://www.ccfs.mq.edu.au/).

\section{REFERENCES}

Adams, C. J., Miller, H., Toselli, A. J., and Griffin, W. L., 2008, The Puncoviscana Formation of northwest Argentina: U-Pb geochronology of detrital zircons and $\mathrm{Rb}-\mathrm{Sr}$ metamorphic ages and their bearing on its stratigraphic age, sediment provenance and tectonic setting: Neues Jahrbuch Fur Geologie Und Palaontologie-Abhandlungen, v. 247, n. 3, p. 341-352, http://dx.doi.org/10.1127/0077-7749/2008/ 0247-0341

Adams, C. J., Miller, H., Acenolaza, F. G., Toselli, A. J., and Griffin, W. L., 2011, The Pacific Gondwana margin in the late Neoproterozoic-early Paleozoic: Detrital zircon U-Pb ages from metasediments in northwest Argentina reveal their maximum age, provenance and tectonic setting: Gondwana Research, v. 19, n. 1, p. 71-83, http://dx.doi.org/10.1016/j.gr.2010.05.002

Altherr, R., Holl, A., Hegner, E., Langer, C., and Kreuzer, H., 2000, High-potassium, calc-alkaline I-type plutonism in the European Variscides: northern Vosges (France) and northern Schwarzwald (Germany): Lithos, v. 50, n. 1-3, p. 51-73, http://dx.doi.org/10.1016/S0024-4937(99)00052-3

Andersen, T., 2002, Correction of common lead in U-Pb analyses that do not report ${ }^{204} \mathrm{~Pb}$ : Chemical Geology, v. 192, n. 1-2, p. 59-79, http://dx.doi.org/10.1016/S0009-2541(02)00195-X

Appleby, S. K., Gillespie, M. R., Graham, C. M., Hinton, R. W., Oliver, G. J. H., Kelly, N. M., and Eimf, 2010, 
Do S-type granites commonly sample infracrustal sources? New results from an integrated $\mathrm{O}, \mathrm{U}-\mathrm{Pb}$ and Hf isotope study of zircon: Contributions to Mineralogy and Petrology, v. 160, n. 1, p. 115-132, http:/ /dx.doi.org/10.1007/s00410-009-0469-3

Barbarin, B., 1999, A review of the relationships between granitoid types, their origins and their geodynamic environments: Lithos, v. 46, n. 3, p. 605-626, http://dx.doi.org/10.1016/S0024-4937(98)00085-1

Barby, B. J., and Gehrels, G., 2006, Detrital zircon reference for the North China block: Journal of Asian Earth Sciences, v. 26, n. 6, p. 637-648, http://dx.doi.org/10.1016/j.jseaes.2004.12.005

Be'eri-Shlevin, Y., Gee, D., Claesson, S., Ladenberger, A., Majka, J., Kirkland, C., Robinson, P., and Frei, D., 2011, Provenance of Neoproterozoic sediments in the Särv nappes (Middle Allochthon) of the Scandinavian Caledonides: LA-ICP-MS and SIMS U-Pb dating of detrital zircons: Precambrian Research, v. 187, n. 1-2, p. 181-200, http://dx.doi.org/10.1016/j.precamres.2011.03.007

Boger, S. D., Carson, C. J., Wilson, C. J. L., and Fanning, C. M., 2000, Neoproterozoic deformation in the Radok Lake region of the northern Prince Charles Mountains East Antarctica; evidence for a single protracted orogenic event: Precambrian Research, v. 104, n. 1-2, p. 1-24, http://dx.doi.org/10.1016/ S0301-9268(00)00079-6

Bonin, B., 2007, A-type granites and related rocks: Evolution of a concept, problems and prospects: Lithos, v. 97, n. 1-2, p. 1-29, http://dx.doi.org/10.1016/j.lithos.2006.12.007

Bouchez, J. L., Delas, C., Gleizes, G., Nédélec, A., and Cuney, M., 1992, Submagmatic Microfractures in Granites: Geology, v. 20, n. 1, p. 35-38, http://dx.doi.org/10.1130/0091-7613(1992)020<0035: SMIG>2.3.CO;2

Cawood, P. A., Nemchin, A. A., Smith, M., and Loewy, S., 2003, Source of the Dalradian Supergroup constrained by U-Pb dating of detrital zircon and implications for the East Laurentian margin: Journal of the Geological Society, v. 160, n. 2, p. 231-246, http://dx.doi.org/10.1144/0016-764902-039

Chappell, B. W., 1999, Aluminium saturation in I- and S-type granites and the characterization of fractionated haplogranites: Lithos, v. 46, n. 3, p. 535-551, http://dx.doi.org/10.1016/S00244937(98)00086-3

Chappell, B. W., and White, A. J. R., 1992, I-Type and S-Type Granites in the Lachlan Fold Belt: Transactions of the Royal Society of Edinburgh-Earth Sciences, v. 83, n. 1-2, p. 1-26, http://dx.doi.org/10.1017/ S0263593300007720

Chen, Z. Y., Yang, S. S., Meng, E. G., and Huang, Z. Q., 2004, Revision of Precambrian stratigraphic units in the Bayan Nuru area, Alxa Zuoqi, Inner Mongolia: Geological Bulletin of China, v. 23, n. 4, p. 345-351 (in Chinese with English abstract).

Collins, W. J., and Richards, S. W., 2008, Geodynamic significance of S-type granites in circum-Pacific orogens: Geology, v. 36, n. 7, p. 559-562, http://dx.doi.org/10.1130/G24658A.1

Dan, W., Li, X. H., Guo, J. H., Liu, Y., and Wang, X. C., 2012a, Integrated in situ zircon U-Pb age and Hf-O isotopes for the Helanshan khondalites in North China Craton: Juvenile crustal materials deposited in active or passive continental margin?: Precambrian Research, v. 222-223, p. 143-158, http://dx.doi.org/ 10.1016/j.precamres.2011.07.016

- 2012b, Paleoproterozoic evolution of the eastern Alxa Block, westernmost North China: Evidence from in situ zircon U-Pb dating and Hf-O isotopes: Gondwana Research, v. 21, n. 4, p. 838-864, http://dx.doi.org/10.1016/j.gr.2011.09.004

Darby, B. J., and Gehrels, G., 2006, Detrital zircon reference for the North China block: Journal of Asian Earth Sciences, v. 26, n. 6, p. 637-648, http://dx.doi.org/10.1016/j.jseaes.2004.12.005

Dong, C. Y., Liu, D. Y., Li, J. J., Wan, Y. S., Zhou, H. Y., Li, C. D., Yang, Y. H., and Xie, L. W., 2007, Palaeoproterozoic Khondalite Belt in the western North China Craton: New evidence from SHRIMP dating and $\mathrm{Hf}$ isotope composition of zircons from metamorphic rocks in the Bayan Ul-Helan Mountains area: Chinese Science Bulletin, v. 52, n. 21, p. 2984-2994, http:/ /dx.doi.org/10.1007/s11434007-0404-9

Eby, G. N., 1990, The A-Type granitoids: A review of their occurrence and chemical characteristics and speculations on their petrogenesis: Lithos, v. 26, n. 1-2, p. 115-134, http://dx.doi.org/10.1016/00244937(90) 90043-Z

Fergusson, C. L., Carr, P. F., Fanning, C. M., and Green, T. J., 2001, Proterozoic-Cambrian detrital zircon and monazite ages from the Anakie Inlier, central Queensland: Grenville and Pacific-Gondwana signatures: Australian Journal of Earth Sciences, v. 48, n. 6, p. 857-866, http://dx.doi.org/10.1046/j.14400952.2001.00904.x

Fergusson, C. L., Henderson, R. A., Fanning, C. M., and Withnall, I. W., 2007, Detrital zircon ages in Neoproterozoic to Ordovician siliciclastic rocks, northeastern Australia: implications for the tectonic history of the East Gondwana continental margin: Journal of the Geological Society, v. 164, n. 1, p. 215-225, http://dx.doi.org/10.1144/0016-76492005-136

Fitzsimons, I. C. W., 2000, Grenville-age basement provinces in East Antarctica: Evidence for three separate collisional orogens: Geology, v. 28, n. 10, p. 879-882, http://dx.doi.org/10.1130/00917613(2000) 28<879:GBPIEA)2.0.CO;2

Gao, Q. L., Zheng, J. P., Zhang, Z. H., Xiong, Q., and Chen, X., 2009, Complex evolution of the southern margin of the North China Craton: Evidence from LA-ICPMS U-Pb dating of zircons in Zhouzhuang meta-gabbro: Acta Petrologica Sinica, v. 25, n. 12, p. 3275-3286.

Geng, Y. S., and Zhou, X. W., 2010, Early Neoproterozoic granite events in Alxa area of Inner Mongolia and their geological significance: evidence from geochronology: Acta Petrologica et Mineralogica, v. 29, p. 779-795 (in Chinese with English abstract).

2011, Characterastics of geochemistry and zircon Hf isotope of the Early Neoproterozoic granite in Alxa area, Inner Mongolia: Acta Petrologica Sinica, v. 27, p. 897-908.

Geng, Y. S., Wang, X. S., Shen, Q. H., and Wu, C. M., 2002, The discovery of Neoproterozoic Jinningian 
deformed granites in Alxa area and its significance: Acta Petrologica et Mineralogica, v. 21, p. 412-420 (in Chinese with English abstract).

2006, Redefinition of the Alxa Group-complex (Precambrian metamorphic basement) in the Alxa area, Inner Mongolia: Geology in China, v. 33, p. 138-145 (in Chinese with English abstract).

2007, Chronology of the Precambrian metamorphic series in the Alxa area, Inner Mongolia: Geology in China, v. 34, p. 251-261 (in Chinese with English abstract).

Geng, Y. S., Wang, X. S., Wu, C. M., and Zhou, X. W., 2010, Late-Paleoproterozoic tectonothermal events of the metamorphic basement in Alxa area: evidence from geochronology: Acta Petrologica Sinica, v. 26, p. $1159-1170$.

Gong, J. H., Zhang, J. X., and Yu, S. Y., 2011, The origin of Longshoushan Group and associated rocks in the southern part of the Alxa block: constraint from LA-ICP-MS U-Pb zircon dating: Acta Petrologica Et Mineralogica, v. 30, p. 795-818.

Gong, J. H., Zhang, J. X., Yu, S. Y., Li, H. K., and Hou, K. J., 2012, Ca. 2.5 Ga TTG rocks in the western Alxa Block and their implications: Chinese Science Bulletin, v. 57, p. 4064-4076.

Goodge, J. W., Williams, I. S., and Myrow, P., 2004, Provenance of Neoproterozoic and lower Paleozoic siliciclastic rocks of the central Ross orogen, Antarctica: Detrital record of rift-, passive-, and activemargin sedimentation: Geological Society of America Bulletin, v. 116, n. 9-10, p. 1253-1279, http:// dx.doi.org/10.1130/B25347.1

Griffin, W. L., Pearson, N. J., Belousova, E. A., and Saeed, A., 2006, Comment: Hf-isotope heterogeneity in zircon 91500: Chemical Geology, v. 233, n. 3-4, p. 358-363, http://dx.doi.org/10.1016/j.chemgeo. 2006.03.007

GSBGMR (Gansu Bureau of Geology and Mineral Resources), 1989, Regional Geology of Gansu Province: Beijing, Geological Publishing House, 692 p. (in Chinese with English abstract).

Guo, J. H., Sun, M., Chen, F. K., and Zhai, M. G., 2005, Sm-Nd and SHRIMP U-Pb zircon geochronology of high-pressure granulites in the Sanggan area, North China Craton: timing of Paleoproterozoic continental collision: Journal of Asian Earth Sciences, v. 24, n. 4, p. 629-642, http:/ /dx.doi.org/10.1016/ j.jseaes.2004.01.017

He, Y. H., Zhao, G. C., Sun, M., and Xia, X. P., 2009, SHRIMP and LA-ICP-MS zircon geochronology of the Xiong'er volcanic rocks: Implications for the Paleo-Mesoproterozoic evolution of the southern margin of the North China Craton: Precambrian Research, v. 168, n. 3-4, p. 213-222, http://dx.doi.org/ 10.1016/j.precamres.2008.09.011

Healy, B., Collins, W. J., and Richards, S. W., 2004, A hybrid origin for Lachlan S-type granites: the Murrumbidgee Batholith example: Lithos, v. 78, n. 1-2, p. 197-216, http://dx.doi.org/10.1016/ j.lithos.2004.04.047

Heaman, L. M., Bowins, R., and Crocket, J., 1990, The Chemical-Composition of Igneous Zircon Suites: Implications for Geochemical Tracer Studies: Geochimica et Cosmochimica Acta, v. 54, n. 6, p. 15971607, http://dx.doi.org/10.1016/0016-7037(90)90394-Z

Hu, B., Zhai, M. G., Guo, J. H., Peng, P., Liu, F., and Liu, S., 2009, LA-ICP-MS U-Pb geochronology of detrital zircons from the Huade Group in the northern margin of the North China Craton and its tectonic significance.: Acta Petrologica Sinica, v. 25, n. 1, p. 193-211.

Huo, F. C., Cao, J. X., Dong, Y. S., Gu, Q. C., and Yan, Z. Q., 1987, The division, correlation, metamorphism and mineralization features of the lower and middle Precambrian in the Helanshan Mountian-Alashan Region, China: Journal of Changchun College of Geology, v. 17, p. 35-48 (in Chinese with English abstract)

Jackson, S. E., Pearson, N. J., Griffin, W. L., and Belousova, E. A., 2004, The application of laser ablation-inductively coupled plasma-mass spectrometry to in situ U-Pb zircon geochronology: Chemical Geology, v. 211, n. 1-2, p. 47-69, http://dx.doi.org/10.1016/j.chemgeo.2004.06.017

Jeon, H., Williams, I. S., and Chappell, B. W., 2012, Magma to mud to magma: Rapid crustal recycling by Permian granite magmatism near the eastern Gondwana margin: Earth and Planetary Science Letters, v. 319-320, p. 104-117, http://dx.doi.org/10.1016/j.epsl.2011.12.010

Keay, S., Steele, D., and Compston, W., 1999, Identifying granite sources by SHRIMP U-Pb zircon geochronology: an application to the Lachlan foldbelt: Contributions to Mineralogy and Petrology, v. 137, n. 4, p. 323-341, http://dx.doi.org/10.1007/s004100050553

Kelly, N. M., Clarke, G. L., and Fanning, C. M., 2002, A two-stage evolution of the Neoproterozoic Rayner structural episode: new $\mathrm{U}-\mathrm{Pb}$ sensitive high resolution ion microprobe constraints from the Oygarden Group, Kemp Land East Antarctica: Precambrian Research, v. 116, n. 3-4, p. 307-330, http:/ /dx.doi.org/ $10.1016 /$ S0301-9268(02)00028-1

Kretz, R., 1983, Symbols for rock-forming minerals: American Mineralogist, v. 68, p. 277-279.

Kröner, A., Wilde, S. A., Li, J. H., and Wang, K. Y., 2005, Age and evolution of a late Archean to Paleoproterozoic upper to lower crustal section in the Wutaishan/Hengshan/Fuping terrain of northern China: Journal of Asian Earth Sciences, v. 24, n. 5, p. 577-595, http://dx.doi.org/10.1016/ j.jseaes.2004.01.001

Kröner, A., Wilde, S. A., Zhao, G. C., O’Brien, P. J., Sun, M., Liu, D. Y., Wan, Y. S., Liu, S. W., and Guo, J. H., 2006, Zircon geochronology and metamorphic evolution of mafic dykes in the Hengshan Complex of northern China: Evidence for late Palaeoproterozoic extension and subsequent high-pressure metamorphism in the North China Craton: Precambrian Research, v. 146, n. 1-2, p. 45-67, http://dx.doi.org/ 10.1016/j.precamres.2006.01.008

Kusky, T. M., 2011, Geophysical and geological tests of tectonic models of the North China Craton: Gondwana Research, v. 20, n. 1, p. 26-35, http://dx.doi.org/10.1016/j.gr.2011.01.004

Kusky, T. M., and Li, J. H., 2003, Paleoproterozoic tectonic evolution of the North China Craton: Journal of Asian Earth Sciences, v. 22, n. 4, p. 383-397, http://dx.doi.org/10.1016/S1367-9120(03)00071-3 
Li, J. J., ms, 2006, Regional Metallogenic system of Alashan Block in Inner Monolia Automomous Region: Beijing, China, China University of Geoscience, Ph. D. thesis, 177 p.

Li, J. Y., Zhang, J., and Qu, J. F., 2012, Amalgamation of North China Craton with Alxa Block in the Late of Early Paleozoic: evidence from sedimentary sequences in the Niushou mountain, Ningxia Hui Autonomous Region, NW China: Geological Review, v. 58, p. 208-214.

Li, Q. L., Li, X. H., Liu, Y., Tang, G. Q., Yang, J. H., and Zhu, W. G., 2010a, Precise U-Pb and Pb-Pb dating of Phanerozoic baddeleyite by SIMS with oxygen flooding technique: Journal of Analytical Atomic Spectrometry, v. 25, p. 1107-1113, http://dx.doi.org/10.1039/b923444f

Li, Q. L., Wu, F. Y., Li, X. H., Qiu, Z. L., Liu, Y., Yang, Y. H., and Tang, G. Q., 2011, Precisely dating Paleozoic kimberlites in the North China Craton and Hf isotopic constraints on the evolution of the subcontinental lithospheric mantle: Lithos, v. 126, n. 1-2, p. 127-134, http://dx.doi.org/10.1016/j.lithos.2011.07.001

Li, X. H., Li, Z. X., Ge, W. C., Zhou, H. W., Li, W. X., Liu, Y., and Wingate, M. T. D., 2003, Neoproterozoic granitoids in South China: crustal melting above a mantle plume at ca. 825 Ma?: Precambrian Research, v. 122, n. 1-4, p. 45-83, http://dx.doi.org/10.1016/S0301-9268(02)00207-3

Li, X. H., Liu, D. Y., Sun, M., Li, W. X., Liang, X. R., and Liu, Y., 2004, Precise Sm-Nd and U-Pb isotopic dating of the supergiant Shizhuyuan polymetallic deposit and its host granite, SE China: Geological Magazine, v. 141, n. 2, p. 225-231, http://dx.doi.org/10.1017/S0016756803008823

Li, X. H., Qi, C. S., Liu, Y., Liang, X. R., Tu, X. L., Xie, L. W., and Yang, Y. H., 2005, Petrogenesis of the Neoproterozoic bimodal volcanic rocks along the western margin of the Yangtze Block: New constraints from Hf isotopes and Fe/Mn ratios: Chinese Science Bulletin, v. 50, p. 2481-2486, http://dx.doi.org/ $10.1360 / 982005-287$

Li, X. H., Li, W. X., and Li, Z. X., 2007, On the genetic classification and tectonic implications of the Early Yanshanian granitoids in the Nanling Range, South China: Chinese Science Bulletin, v. 52, n. 14, p. 1873-1885, http://dx.doi.org/10.1007/s11434-007-0259-0

Li, X. H., Liu, Y., Li, Q. L., Guo, C. H., and Chamberlain, K. R., 2009a, Precise determination of Phanerozoic zircon $\mathrm{Pb} / \mathrm{Pb}$ age by multicollector SIMS without external standardization: Geochemistry, Geophysics, Geosystems, v. 10, n. 4, Q04010, http://dx.doi.org/10.1029/2009GC002400

Li, X. H., Li, W. X., Li, Z. X., Lo, C. H., Wang, J., Ye, M. F., and Yang, Y. H., 2009b, Amalgamation between the Yangtze and Cathaysia Blocks in South China: Constraints from SHRIMP U-Pb zircon ages, geochemistry and Nd-Hf isotopes of the Shuangxiwu volcanic rocks: Precambrian Research, v. 174, n. 1-2, p. 117-128, http://dx.doi.org/10.1016/j.precamres.2009.07.004

Li, X. H., Li, W. X., Li, Q. L., Wang, X. C., Liu, Y., and Yang, Y. H., 2010b, Petrogenesis and tectonic significance $\sim 850 \mathrm{Ma}$ Gangbian alkaline complex in South China: Evidence from in situ zircon U-Pb dating, Hf-O isotopes and whole-rock geochemistry: Lithos, v. 114, n. 1-2, p. 1-15, http://dx.doi.org/ 10.1016/j.lithos.2009.07.011

Li, X. H., Long, W. G., Li, Q. L., Liu, Y., Zheng, Y. F., Yang, Y. H., Chamberlain, K. R., Wan, D. F., Guo, C. H., Wang, X. C., and Tao, H., 2010c, Penglai Zircon Megacrysts: A Potential New Working Reference Material for Microbeam Determination of Hf-O Isotopes and U-Pb Age: Geostandards and Geoanalytical Research, v. 34, n. 2, p. 117-134, http://dx.doi.org/10.1111/j.1751-908X.2010.00036.x

Li, Z. X., Bogdanova, S. V., Collins, A. S., Davidson, A., De Waele, B., Ernst, R. E., Fitzsimons, I. C. W., Fuck, R. A., Gladkochub, D. P., Jacobs, J., Karlstrom, K. E., Lu, S., Natapov, L. M., Pease, V., Pisarevsky, S. A., Thrane, K., and Vernikovsky, V., 2008, Assembly, configuration, and break-up history of Rodinia: A synthesis: Precambrian Research, v. 160, n. 1-2, p. 179-210, http://dx.doi.org/10.1016/j.precamres. 2007.04.021

Liu, S. A., Li, S. G., He, Y. S., and Huang, F., 2010a, Geochemical contrasts between early Cretaceous ore-bearing and ore-barren high-Mg adakites in central-eastern China: Implications for petrogenesis and $\mathrm{Cu}-\mathrm{Au}$ mineralization: Geochimica et Cosmochimica Acta, v. 74, n. 24, p. 7160-7178, http:// dx.doi.org/10.1016/j.gca.2010.09.003

Liu, Y. Q., Gao, L. Z., Liu, Y. X., Song, B., and Wang, Z. X., 2005, Zircon U-Pb dating for the early Neoproterozoic mafic magmatism in Xuhuai area: Chinese Science Bulletin, v. 50, p. 2514-2521.

Liu, Y. S., Gao, S., Hu, Z. C., Gao, C. G., Zong, K. Q., and Wang, D. B., 2010b, Continental and Oceanic Crust Recycling-induced Melt-Peridotite Interactions in the Trans-North China Orogen: U-Pb Dating, Hf Isotopes and Trace Elements in Zircons from Mantle Xenoliths: Journal of Petrology, v. 51, n. 1-2, p. 537-571, http://dx.doi.org/10.1093/petrology/egp082

Ludwig, K. R., 2003, Users Manual for Isoplot 3.00: A Geochronological Toolkit for Microsoft Excel: Berkeley Geochronology Center, Special Publication 4, 70 p.

Menzies, M. A., Fan, W. M., and Zhang, M., 1993, Palaeozoic and Cenozoic lithoprobe and the loss of $>120$ $\mathrm{km}$ of Archean lithosphere, Sino-Korean craton, China, in Prichard, H. M., Alabaster, T., Harris, N. B. W., and Neary, C. R., editors, Magmatic Processes and Plate Tectonics: Geological Society, London, Special Publications, v. 76, p. 71-81, http://dx.doi.org/10.1144/GSL.SP.1993.076.01.04

Mezger, K., and Cosca, M. A., 1999, The thermal history of the Eastern Ghats Belt (India) as revealed by $\mathrm{U}-\mathrm{Pb}$ and ${ }^{40} \mathrm{Ar} /{ }^{39} \mathrm{Ar}$ dating of metamorphic and magmatic minerals; implications for the SWEAT correlation: Precambrian Research, v. 94, n. 3-4, p. 251-271, http://dx.doi.org/10.1016/S03019268(98)00118-1

Misra, S. N., 1971, Chemical distinction of high-grade ortho- and para-metabasites: Norsk Geologisk Tidsskrift, v. 51, p. 311-316.

Morel, M. L. A., Nebel, O., Nebel-Jacobsen, Y. J., Miller, J. S., and Vroon, P. Z., 2008, Hafnium isotope characterization of the GJ-1 zircon reference material by solution and laser-ablation MC-ICPMS: Chemical Geology, v. 255, n. 1-2, p. 231-235, http://dx.doi.org/10.1016/j.chemgeo.2008.06.040

NMBGMR (Nei Mongol Bureau of Geology and Mineral Resources), 1991, Regional Geology of Nei Mongol Autonomous Region: Beijing, Geological Publishing House, 725 p. (in Chinese with English abstract). 
O'Neil, J. R., and Chappell, B. W., 1977, Oxygen and hydrogen isotope relations in the Berridale batholith: Journal of the Geological Society, v. 133, n. 6, p. 559-571, http://dx.doi.org/10.1144/gsjgs.133.6.0559

Paterson, S. R., Vernon, R. H., and Tobisch, O. T., 1989, A Review of Criteria for the Identification of Magmatic and Tectonic Foliations in Granitoids: Journal of Structural Geology, v. 11, n. 3, p. 349-363, http://dx.doi.org/10.1016/0191-8141(89)90074-6

Peng, P., Zhai, M. G., Zhang, H. F., and Guo, J. H., 2005, Geochronological constraints on the Paleoproterozoic evolution of the North China craton: SHRIMP zircon ages of different types of Mafic dikes: International Geology Review, v. 47, n. 5, p. 492-508, http://dx.doi.org/10.2747/0020-6814.47.5.492

Peng, P., Zhai, M. G., and Guo, J. H., 2006, 1.80-1.75 Ga mafic dyke swarms in the central North China craton: implications for a plume related break-up event, in Hanski, E., Mertanen, S., Ramö, T., and Vuollo, J., editors, Dyke Swarms-Time Markers of Crustal Evolution: London, Taylor \& Francis Publisher, p. 99-112, http://dx.doi.org/10.1201/NOE0415398992.ch7

Peng, P., Zhai, M. G., Ernst, R. E., Guo, J. H., Liu, F., and Hu, B., 2008, A 1.78 Ga large igneous province in the North China craton: The Xiong'er volcanic province and the North China dyke Swarm: Lithos, v. 101, n. 3-4, p. 260-280, http://dx.doi.org/10.1016/j.lithos.2007.07.006

Peng, P., Bleeker, W., Ernst, R. E., Söderlund, U., and McNicoll, V., 2011a, U-Pb baddeleyite ages, distribution and geochemistry of 925 Ma mafic dykes and 900 Ma sills in the North China craton: Evidence for a Neoproterozoic mantle plume: Lithos, v. 127, n. 1-2, p. 210-221, http://dx.doi.org/ 10.1016/j.lithos.2011.08.018

Peng, P., Zhai, M. G., Li, Q. L., Wu, F. Y., Hou, Q. L., Li, Z., Li, T. S., and Zhang, Y. B., 2011b, Neoproterozoic $(\sim 900 \mathrm{Ma})$ Sariwon sills in North Korea: Geochronology, geochemistry and implications for the evolution of the south-eastern margin of the North China Craton: Gondwana Research, v. 20, n. 1, p. 243-254, http://dx.doi.org/10.1016/j.gr.2010.12.011

Peng, R. M., Zhai, Y. S., Wang, J. P., Chen, X. F., Liu, Q., Lv, J. F., Shi, Y. X.,Wang, G., Li, S. W., Wang, L. G., Ma, Y. T., and Zhang, P., 2010, Discovery of Neoproterozoic acid volcanic rock in the south-western section of Langshan, Inner Mongolia: Chinese Science Bulletin, v. 55, p. 2611-2620, http:/ / dx.doi.org/ $10.1360 / 972010-266$

Ren, J. S., Jiang, C. F., Zhang, Z. K., and Qin, D. Y., 1987, Geotectonic evolution of China: Berlin, Science Press, Springer-Verlag, 203 p.

Rollinson, H. R., 1993, Using Geochemical Data: Evaluation, Presentation, Interpretation: London, Longman Geochemistry Series, 352 p.

Shaw, D. M., 1972, Origin of Apsley Gneiss, Ontario: Canadian Journal of Earth Sciences, v. 9, n. 1, p. 18-35, http://dx.doi.org/10.1139/e72-002

Shu, L. S., Deng, X. L., Zhu, W. B., Ma, D. S., and Xiao, W. J., 2011, Precambrian tectonic evolution of the Tarim Block, NW China: New geochronological insights from the Quruqtagh domain: Journal of Asian Earth Sciences, v. 42, n. 5, p. 774-790, http://dx.doi.org/10.1016/j.jseaes.2010.08.018

Slama, J., Kosler, J., Condon, D. J., Crowley, J. L., Gerdes, A., Hanchar, J. M., Horstwood, M. S. A., Morris, G. A., Nasdala, L., Norberg, N., Schaltegger, U., Schoene, B., Tubrett, M. N., and Whitehouse, M. J., 2008, Plešovice zircon-A new natural reference material for U-Pb and $\mathrm{Hf}$ isotopic microanalysis: Chemical Geology, v. 249, n. 1-2, p. 1-35, http://dx.doi.org/10.1016/i.chemgeo.2007.11.005

Söderlund, U., Patchett, J. P., Vervoort, J. D., and Isachsen, C. E., 2004, The ${ }^{176}$ Lu decay constant determined by $\mathrm{Lu}-\mathrm{Hf}$ and $\mathrm{U}-\mathrm{Pb}$ isotope systematics of Precambrian mafic intrusions: Earth and Planetary Science Letters, v. 219, n. 3-4, p. 311-324, http://dx.doi.org/10.1016/S0012-821X(04)00012-3

Stacey, J. S., and Kramers, J. D., 1975, Approximation of Terrestrial Lead Isotope Evolution by a 2-Stage Model: Earth and Planetary Science Letters, v. 26, n. 2, p. 207-221, http://dx.doi.org/10.1016/0012821X(75)90088-6

Sun, J. F., Yang, J. H., Wu, F. Y., and Wilde, S. A., 2012, Precambrian crustal evolution of the eastern North China Craton as revealed by U-Pb ages and $\mathrm{Hf}$ isotopes of detrital zircons from the Proterozoic Jing'eryu Formation: Precambrian Research, v. 200-203, p. 184-208, http://dx.doi.org/10.1016/j.precamres. 2012.01.018

Sun, S. S., and McDonough, W. F., 1989, Chemical and isotopic systematics of oceanic basalt: implications for mantle composition and processes, in Sanders, A. D., and Norry, M. J., editiors, Magmatism in the Ocean Basins: Geological Society, London, Special Publications, v. 42, p. 313-345, http://dx.doi.org/ 10.1144/GSL.SP.1989.042.01.19

Sun, W. H., Zhou, M. F., Gao, J. F., Yang, Y. H., Zhao, X. F., and Zhao, J. H., 2009, Detrital zircon U-Pb geochronological and Lu-Hf isotopic constraints on the Precambrian magmatic and crustal evolution of the western Yangtze Block, SW China: Precambrian Research, v. 172, n. 1-2, p. 99-126, http:// dx.doi.org/10.1016/j.precamres.2009.03.010

Tung, K. A., Yang, H. Y., Liu, D. Y., Zhang, J. X., Tseng, C. Y., and Wan, Y. S., 2007, SHRIMP U-Pb geochronology of the detrital zircons from the Longshoushan Group and its tectonic significance: Chinese Science Bulletin, v. 52, n. 10, p. 1414-1425, http://dx.doi.org/10.1007/s11434-007-0189-x

Valley, J. W., Lackey, J. S., Cavosie, A. J., Clechenko, C. C., Spicuzza, M. J., Basei, M. A. S., Bindeman, I. N., Ferreira, V. P., Sial, A. N., King, E. M., Peck, W. H., Sinha, A. K., and Wei, C. S., 2005, 4.4 billion years of crustal maturation: oxygen isotope ratios of magmatic zircon: Contributions to Mineralogy and Petrology, v. 150, n. 6, p. 561-580, http://dx.doi.org/10.1007/s00410-005-0025-8

Wan, Y. S., Liu, D. Y., Wang, W., Song, T. R., Kroner, A., Dong, C. Y., Zhou, H. Y., and Yin, X. Y., 2011, Provenance of Meso- to Neoproterozoic cover sediments at the Ming Tombs, Beijing, North China Craton: An integrated study of U-Pb dating and $\mathrm{Hf}$ isotopic measurement of detrital zircons and whole-rock geochemistry: Gondwana Research, v. 20, n. 1, p. 219-242, http://dx.doi.org/10.1016/ j.gr.2011.02.009

Wang, L. J., Griffin, W. L., Yu, J. H., and O'Reilly, S. Y., 2010, Precambrian crustal evolution of the Yangtze 
Block tracked by detrital zircons from Neoproterozoic sedimentary rocks: Precambrian Research, v. 177, n. 1-2, p. 131-144, http://dx.doi.org/10.1016/j.precamres.2009.11.008

Wang, Q. H., Yang, D. B., and Xu, W. L., 2012a, Neoproterozoic basic magmatism in the southeast margin of North China Craton: Evidence from whole-rock geochemistry, U-Pb and Hf isotopic study of zircons from diabase swarms in the Xuzhou-Huaibei area: Science China: Earth Science, http://dx.doi.org/ $10.1007 / \mathrm{s} 11430-011-4237-7$.

Wang, X. C., Li, X. H., Li, Z. X., Li, Q. L., Tang, G. Q., Gao, Y. Y., Zhang, Q. R., and Liu, Y., 2012b. Episodic Precambrian crust growth: Evidence from U-Pb ages and $\mathrm{Hf}-\mathrm{O}$ isotopes of zircon in the Nanhua Basin, central South China: Precambrian Research, v. 222-223, p. 386-403, http://dx.doi.org/10.1016/ j.precamres.2011.06.001

Wang, X. L., Jiang, S. Y., Dai, B. Z., Griffin, W. L., Dai, M. N., and Yang, Y. H., 2011, Age, geochemistry and tectonic setting of the Neoproterozoic ( $c a .830 \mathrm{Ma}$ ) gabbros on the southern margin of the North China Craton: Precambrian Research, v. 190, n. 1-4, p. 35-47, http://dx.doi.org/10.1016/j.precamres. 2011.08.004

Wiedenbeck, M., Alle, P., Corfu, F., Griffin, W. L., Meier, M., Oberli, F., Von Quadt, A., Roddick, J. C., and Speigel, W., 1995, Three Natural Zircon Standards for U-Th-Pb, Lu-Hf, Trace-Element and REE Analyses: Geostandards Newsletter, v. 19, n. 1, p. 1-23, http://dx.doi.org/10.1111/j.1751908X.1995.tb00147.x

Wiedenbeck, M., Hanchar, J. M., Peck, W. H., Sylvester, P., Valley, J., Whitehouse, M., Kronz, A., Morishita, Y., Nasdala, L., Fiebig, J., Franchi, I., Girard, J. P., Greenwood, R. C., Hinton, R., Kita, N., Mason, P. R. D., Norman, M., Ogasawara, M., Piccoli, R. M., Rhede, D., Satoh, H., Schulz-Dobrick, B., Skar, O., Spicuzza, M. J., Terada, K., Tindle, A., Togashi, S., Vennemann, T., Xie, Q., and Zheng, Y. F., 2004, Further characterisation of the 91500 zircon crystal: Geostandards and Geoanalytical Research, v. 28, n. 1, p. 9-39, http://dx.doi.org/10.1111/j.1751-908X.2004.tb01041.x

Woodhead, J. D., and Hergt, J. M., 2005, A preliminary appraisal of seven natural zircon reference materials for in situ Hf isotope determination: Geostandards and Geoanalytical Research, v. 29, n. 2, p. 183-195, http://dx.doi.org/10.1111/j.1751-908X.2005.tb00891.x

Wu, F. Y., Yang, Y. H., Xie, L. W., Yang, J. H., and Xu, P., 2006, Hf isotopic compositions of the standard zircons and baddeleyites used in U-Pb geochronology: Chemical Geology, v. 234, n. 1-2, p. 105-126, http:/ / dx.doi.org/10.1016/j.chemgeo.2006.05.003

Wu, F. Y., Xu, Y. G., Gao, S., and Zheng, J. P., 2008, Lithospheric thinning and destruction of the North China Craton: Acta Petrologica Sinica, v. 24, p. 1145-1174.

Wu, J. S., Geng, Y. S., Shen, Q. H., Wan, Y. S., Liu, D. Y., and Song, B., 1998, Archean geological characteristics and tectonic evolution of China-Korea Paleo-continent: Beijing, Geological Publishing House, 212 p. (in Chinese).

Xia, X. P., Sun, M., Zhao, G. C., Wu, F. Y., Xu, P., Zhang, J. H., and Luo, Y., 2006, U-Pb and Hf isotopic study of detrital zircons from the Wulashan khondalites: Constraints on the evolution of the Ordos Terrane, western block of the North China Craton: Earth and Planetary Science Letters, v. 241, n. 3-4, p. 581-593, http://dx.doi.org/10.1016/j.epsl.2005.11.024

Xie, L. W., Zhang, Y. B., Zhang, H. H., Sun, J. F., and Wu, F. Y., 2008, In situ simultaneous determination of trace elements, U-Pb and Lu-Hf isotopes in zircon and baddeleyite: Chinese Science Bulletin, v. 53, n. 10, p. 1565-1573, http://dx.doi.org/10.1007/s11434-008-0086-y

Yang, J. H., Wu, F. Y., Wilde, S. A., Belousova, E., and Griffin, W. L., 2008, Mesozoic decratonization of the North China block: Geology, v. 36, n. 6, p. 467-470, http://dx.doi.org/10.1130/G24518A.1

Yang, K. F., Fan, H. R., Santosh, M., Hu, F. F., and Wang, K. Y., 2011, Mesoproterozoic mafic and carbonatitic dykes from the northern margin of the North China Craton: Implications for the final breakup of Columbia supercontinent: Tectonophysics, v. 498, n. 1-4, p. 1-10, http://dx.doi.org/10.1016/ j.tecto.2010.11.015

Ye, M. F., Li, X. H., Li, W. X., Liu, Y., and Li, Z. X., 2007., SHRIMP zircon U-Pb geochronological and whole-rock geochemical evidence for an early Neoproterozoic Sibaoan magmatic arc along the southeastern margin of the Yangtze Block: Gondwana Research, v. 12, n. 1-2, p. 144-156, http:// dx.doi.org/10.1016/j.gr.2006.09.001

Yin, C. Q., Zhao, G. C., Sun, M., Xia, X. P., Wei, C. J., Zhou, X. W., and Leung, W. H., 2009, LA-ICP-MS U-Pb zircon ages of the Qianlishan Complex: Constrains on the evolution of the Khondalite Belt in the Western Block of the North China Craton: Precambrian Research, v. 174, n. 1-2, p. 78-94, http:// dx.doi.org/10.1016/j.precamres.2009.06.008

Yu, J. H., O'Reilly, S. Y., Wang, L. J., Griffin, W. L., Zhang, M., Wang, R. C., Jiang, S. Y., and Shu, L. S., 2008, Where was South China in the Rodinia supercontinent? Evidence from U-Pb geochronology and Hf isotopes of detrital zircons: Precambrian Research, v. 164, n. 1-2, p. 1-15, http://dx.doi.org/10.1016/ j.precamres.2008.03.002

Yu, J. H., O'Reilly, S. Y., Wang, L. J., Griffin, W. L., Zhou, M. F., Zhang, M., and Shu, L. S., 2010, Components and episodic growth of Precambrian crust in the Cathaysia Block South China: Evidence from U-Pb ages and Hf isotopes of zircons in Neoproterozoic sediments: Precambrian Research, v. 181, n. 1-4, p. 97-114, http://dx.doi.org/10.1016/j.precamres.2010.05.016

Zhai, M. G., and Santosh, M., 2011, The early Precambrian odyssey of the North China Craton: A synoptic overview: Gondwana Research, v. 20, n. 1, p. 6-25, http://dx.doi.org/10.1016/j.gr.2011.02.005

Zhai, M. G., Bian, A. G., and Zhao, T. P., 2000, The amalgamation of the supercontinent of North China Craton at the end of Neo-Archaean and its breakup during late Palaeoproterozoic and MesoProterozoic: Science in China Series D-Earth Sciences, v. 43, n. 1, p. 219-232, http:/ /dx.doi.org/10.1007/ BF02911947

Zhang, J., Li, J. Y., Liu, J. F., and Feng, Q. W., 2011, Detrital zircon U-Pb ages of Middle Ordovician flysch sandstones in the western ordos margin: New constraints on their provenances, and tectonic implica- 
tions: Journal of Asian Earth Sciences, v. 42, n. 5, p. 1030-1047, http://dx.doi.org/10.1016/ j.jseaes.2011.03.009

Zhang, J., Li, J. Y., Liu, J. F., Li, Y. F., Qu, J. F., and Feng, Q. W., 2012, The relationship between the Alxa Block and the North China Plate during the early Paleozoic: new information from the middle Ordovician detrital zircon ages in the eastern Alxa Block: Acta Petrologica Sinica, v. 28, p. 2912-2934.

Zhang, S. H., Zhao, Y., Yang, Z. Y., He, Z. F., and Wu, H., 2009, The 1.35 Ga diabase sills from the northern North China Craton: Implications for breakup of the Columbia (Nuna) supercontinent: Earth and Planetary Science Letters, v. 288, n. 3-4, p. 588-600, http://dx.doi.org/10.1016/j.epsl.2009.10.023

Zhao, G. C., 2009, Metamorphic evolution of major tectonic units in the basement of the North China Craton: Key issues and discussion.: Acta Petrologica Sinica, v. 25, p. 1772-1792.

Zhao, G. C., Wilde, S. A., Cawood, P. A., and Sun, M., 2001, Archean blocks and their boundaries in the North China Craton: lithological, geochemical, structural and $P-T$ path constraints and tectonic evolution: Precambrian Research, v. 107, n. 1-2, p. 45-73, http://dx.doi.org/10.1016/S03019268(00)00154-6

Zhao, G. C., Sun, M., Wilde, S. A., and Li, S. Z., 2005, Late Archean to Paleoproterozoic evolution of the North China Craton: key issues revisited: Precambrian Research, v. 136, n. 2, p. 177-202, http:// dx.doi.org/10.1016/j.precamres.2004.10.002

Zhao, T. P., Zhou, M. F., Zhai, M. G., and Xia, B., 2002, Paleoproterozoic rift-related volcanism of the Xiong'er Group, North China Craton: Implications for the breakup of Columbia: International Geology Review, v. 44, n. 4, p. 336-351, http://dx.doi.org/10.2747/0020-6814.44.4.336

Zhu, D. C., Mo, X. X., Niu, Y. L., Zhao, Z. D., Wang, L. Q., Pan, G. T., and Wu, F. Y., 2009, Zircon U-Pb dating and in-situ Hf isotopic analysis of Permian peraluminous granite in the Lhasa terrane, southern Tibet: Implications for Permian collisional orogeny and paleogeography: Tectonophysics, v. 469, n. 1-4, p. 48-60, http://dx.doi.org/10.1016/j.tecto.2009.01.017

Zhu, W. B., Zheng, B. H., Shu, L. S., Ma, D. S., Wu, H. L., Li, Y. X., Huang, W. T., and Yu, J. J., 2011, Neoproterozoic tectonic evolution of the Precambrian Aksu blueschist terrane, northwestern Tarim, China: Insights from LA-ICP-MS zircon U-Pb ages and geochemical data: Precambrian Research, v. 185, n. 3-4, p. 215-230, http://dx.doi.org/10.1016/j.precamres.2011.01.012 